\title{
An Account of the Researches on Races of Herrings Carried out by the Marine Biological Association at Plymouth, 1914-15.
}

By

\author{
J. H. Orton, D.Sc.,
}

Naturalist at the Plymouth Laboratory.

With Figs. 1 to 6 in the Text.

Investigations made
The characters studied
Method of work

[The following Tables record the measurement and enumeration of a number of characters in certain samples of Herrings taken in the neighbourhood of Plymouth. The work was carried out as part of a general scheme for studying the question of the existence of local races of herrings around the British Coasts, which was organised by the Board of Agriculture and Fisheries. In consequence of the war there is no immediate prospect of the figures being analysed and compared with similar figures relating to fish obtained in other localities. It has therefore been thought 
advisable to place them on record as they stand, so that they may be available for other workers at any time. The short explanatory statement of the methods employed was prepared by Dr. Orton, who had charge of most of the work, to accompany the figures when they were sent to the Board of Agriculture and Fisheries, and was not written by him with a view to publication. The drawings have been made by Mrs. Sexton.-E. J. Allen.]

\section{THE INVESTIGATIONS MADE.}

IN accordance with the general scheme of the Board of Agriculture and Fisheries two samples of herrings each of more than 500 specimens have been examined. In the season of 1914-15 we were able to examine in such numbers only the herrings spawning near Plymouth, i.e. in the locality of Bigbury Bay. In December, 1914, a sample of 550 herrings of the shoal spawning in this area was examined in all the characters recommended by the Board, and in January, 1915, a further sample of 525 fish from the same locality was investigated similarly.

In early December, 1914, a small sample of herrings from Cawsand Bay was examined for the purpose of practice and also for comparison with fish from the spawning grounds.

Along with the investigations mentioned above are submitted particulars of a sample of 84 herrings taken in the Channel and examined by Mr. R. S. Clark in July, 1914.

\section{THE CHARACTERS STUDIED.}

The following is the scheme of the characters studied, as authorised by the Board of Agriculture and Fisheries:-

All measurements* are to be made with the special apparatus supplied by the Board. The fish should be placed upon the board in such a way that the snout is pressed against the end board sufficiently hard to keep the mouth shut and the body of the fish should be at right angles to the end board. The measurements are to be in all cases the shortest distance from the end board to each point specified. They are to be in the order given below, and tabulated in this order on the forms supplied. A diagram of the herring is appended (Fig. 1), showing the measurements to be taken.

The measurements required are as follows :-

From the end board to

(1) Nearest point of bony orbit.

(2) Hinder edge of operculum.

* Measurements are all given in centimetres. 
(3) Anterior end of dorsal fin.

(4) Base of pelvic fins.

(5) Posterior end of dorsal fin.

(6) Anterior end of anal fin.

(7) Posterior edge of hindmost scale.*

(8) Distal end of mid-caudal ray.

(9) Distal end of longest ray in dorsal fluke of caudal fin, when fluke is placed so that its dorsal margin lies parallel to line of measurement (i.e. line on board upon which snout and mid-caudal ray should lie).

These measurements made, the following are counted :-

(10) Number of keeled scales on median ventral line in front of base of pelvic fins. [This character was found unreliable and was omitted.]

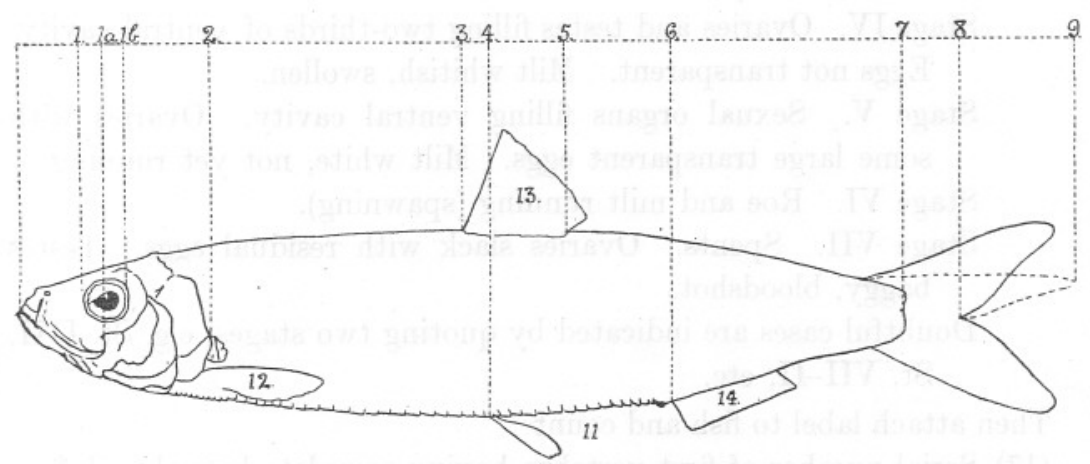

FIG. 1.-Diagram of Herring, showing the measurements, etc., taken by Dr. Orton, copied from the figure supplied by the Board of Agriculture and Fisheries.

(11) Number of keeled scales between base of pelvics and anus.

(12) Number of rays in right pectoral fin.

(13) Number of rays in dorsal fin.

(14) Number of rays in anal fin.

Then take

(15) Weight of fish in apparatus supplied $\dagger$ to nearest 10 grms.

Then

Take scales $\$$ from neighbourhood of pectoral fins and preserve in envelopes to be examined by Hjort's method.

Then open fish and take

(16) Sex and degree of maturity (Hjort's scale).

* See p. 83.

$\dagger$ Each fish was weighed singly to the nearest gram on an ordinary balance in our work.

$\ddagger$ The scales were forwarded to the Board for examination. The pyloric cæca of eac fish were also preserved (see p. 86). 
State of sexual organs is classified in 7 stages (Publications de Circonstance, No. 53, p. 35).

Stage I. Virgin individuals. Very small sexual organs close under vertebral column. ㅇ wine-coloured torpedo-shaped ovaries about $2-3 \mathrm{~cm}$. long and $2-3 \mathrm{~mm}$. thick. Eggs invisible to naked eye. $\widehat{\delta}$ whitish or greyish-brown knife-shaped testes $2-3 \mathrm{~cm}$. long and $2-3 \mathrm{~mm}$. broad.

Stage II. Maturing virgins or recovering spents. Ovaries somewhat longer than half the length of ventral cavity, about $1 \mathrm{~cm}$. diam. Eggs small but visible to naked eye. Milt whitish, somewhat bloodshot, same size as ovaries, but still thin and knifeshaped.

Stage III. Sexual organs more swollen, occupying about half of ventral cavity.

Stage IV. Ovaries and testes filling two-thirds of ventral cavity. Eggs not transparent. Milt whitish, swollen.

Stage V. Sexual organs filling ventral cavity. Ovaries with some large transparent eggs. Milt white, not yet running.

Stage VI. Roe and milt running (spawning).

Stage VII. Spents. Ovaries slack with residual eggs. Testes baggy, bloodshot.

Doubtful cases are indicated by quoting two stages, e.g. St. I-II, St. VII-II, etc.

Then attach label to fish and count

(17) Serial number of first vertebra having complete hæmal arch.*

(18) Total number of vertebræ.

\section{METHOD OF WORK.}

The measurements and weighings of the fish were first made and the counting of the scales and fin-rays accomplished in a second series of operations, and finally the skeletons were prepared in a third stage.

It was found possible to take the weight and measurements of only 250 fish in the first sample and 300 fish in the second sample within 24 hours of the landing of the fish, but weights and measurements of the whole samples of 550 and 525 respectively were completed within less than 36 hours of the landing of the fish. The fish not examined on the first day were kept in an ice chamber until required and remained in good preservation. It may here be noted that both the larger samples were obtained from steam-drifters, on which boats it appears that the fish are subjected to rougher handling than on sailing drifters. Some fish

* The figures given in Tables II, III, and IV denote the number of vertebræ with perfect and imperfect hæmal arches. For details see pp. 80-82. 
were damaged with respect to one or more of the characters required, and were rejected ; apart from these damaged fish there was no other selection effected.

Both larger samples were samples from a large haul of fish and were taken at random from the catch.

The method of work in detail was as follows :-

\section{1st series of operations.}

The fish were first weighed singly and a sample of the scales taken from the region under the pectoral fin and put in a previously numbered envelope. A light metal label attached to a small safety pin was then stuck into the fish, which was passed on to be measured, the weight of the fish in the meantime being called out to the recorder. The measurements 1 to 9 were then taken-being called out and recorded successively. The sex and condition of the gonad were next determined and recorded and the fish finally labelled-by pinning the label to the skull through the orbit-and put into an ice-chest tray. Four persons were concerned in this operation - one to weigh and take scales, one to record, one to hand the fish on and assist mechanically with the measuring, and one to measure and take condition of gonad and sex. It was found that weighing and taking scales could be done on the whole rather more quickly than taking measurements and sex. In this way from about 34 to 44 fish could be examined in one hour's continuous work. The time within which a given number of fish were examined was noted and is given in the account of the examination of the different samples.

\section{2nd series of operations : Counting.}

In the large samples the counting of the keeled scales and fin rays (Characters 11 to 14) began on the third day of the investigation and was finished on the fourth. When four workers were available each counted the same fin - or the scales - in all the fish, and handed each fish on to his or her neighbour in turn. In the early stages of the work each worker called out the count to be recorded, and later each worker kept a record to check the count called out, but in the whole of the second sample, each worker simply recorded his or her own work. When the Characters 11 to 14 had been recorded the alimentary canal was taken out and labelled with the number of the fish, and preserved for the future examination of the pyloric cæca.

\section{3rd stage : Preparing skeletons.}

It was found better to place the fish in cold water, to bring the water to the boil, and allow to boil only two minutes than to boil for ten minutes. 
Not more than upwards to about 50 fish were boiled at a time, and a shallow tray which just fits into the fish kettle was used for containing the fish during boiling, one tray being used for boiling while the boiled fish in another tray were being cleaned.

It was found that with one worker cleaning the fish roughly, another worker could clean up to 30 skeletons in an hour after a little practice. The skeletons of the whole sample in each case were prepared in two working days by one worker cleaning them roughly and two others cleaning them finally. All the skeletons have been kept with their own label for future reference and comparison with others. It was found important not to clean the skeletons too well in the region in front of the anterior complete hæmal arches, and to cut the vertebral artery at an early stage in the cleaning operation. The prepared skeletons were kept in shallow wooden trays.

\section{DISTRIBUTION OF WORK AMONG THE WORKERS.}

The work in the different stages was accomplished with the help of workers who gave their services at different times. The responsibility for the method and form of the work was undertaken by Dr. Orton, but the assistance rendered by the team of helpers can best be shown in tabular form as follows :-

Weighing and taking scales

Recording

Measuring characters 1 to 9 and recording sex and condition of gonad

Counting rays in pectoral fin .

Counting rays in dorsal fin

Counting rays in anal fin

Counting keeled scales between pelvic and anal fins .

Preparing skeletons

Counting vertebræ

Checking counting of vertebræ
Mr. A. J. Smith.

Miss Clark, Mrs. Matthews, Dr. Allen.

\section{Dr. Orton.}

Mr. A. J. Smith, Dr. Orton.

Dr. Allen, Mr. Crawshay, Dr. Orton.

Mrs. Orton, Mrs. Matthews, Dr. Allen, Dr. Orton.

\section{Dr. Orton.}

Dr. Orton, Mr. Smith.

Dr. Orton.

Mrs. Orton, Mrs. Matthews, Dr. Allen.

Mr. William Searle assisted in handling and labelling the fish and labelled the gut with attached pyloric cæca for further examination.

It may be mentioned that a fair amount of practice in measuring, weighing, recording, and counting was done by Dr. Orton, Mr. Smith, Miss Clark, and Mrs. Orton before the large samples were investigated. 


\section{DESCRIPTION OF THE WORKING OF THE SEPARATE SAMPLES.}

Four samples of herrings have been investigated fully, two smal samples and two large ones. For the sake of convenience they have been numbered in chronological order.

Sample I. 84 herrings 9 miles S. of Looe, July 15, 1914.

, II. 32 „, from Cawsand Bay, Dec. 9, 1914.

, III. 550 „, from 6 miles W. by S. of Start Point, Dec. 15, 1914.

, IV. 525 ,, from about 8 miles W.S.W. to about 3 miles S.S.W. of Start Point, Jan. 6, 1915.

\section{Sample I.}

Particulars of this sample are given on the recording sheets. Characters 13 and 14 are given as totals. This sample, being a batch of summer herrings from the Plymouth district, should be specially interesting in comparison with the winter spawning herring; it was examined by Mr. R. S. Clark, with the assistance of Mr. E. Ford and Mr. F. M. Gossen.

\section{Sample II.}

This sample of 32 fish from a total catch of from 250 to 300 was taken on December 9, 1914, from drift nets moored in Cawsand Bay. The fish were in excellent condition and were weighed and measured during the morning of December 9 .

In this sample two additional characters to those recommended by the Board were investigated, namely, $(a)$ the position of the posterior border of the maxilla in relation to the position of the eye, and $(b)$ the number of pyloric cæca. The former necessitated two additional measurements, which were numbered " $1 a$ " and " $1 b . " 1 a$ is the shortest distance between a line tangent to the posterior border of the maxilla taken at right angles to the long axis of the fish, and a line tangent to the tip of the lower jaw at right angles to the long axis of the fish.

$1 b$ is the shortest distance between a tangent to the posterior border of the orbit taken at right angles to the long axis of the fish, and a similar tangent to the tip of the lower jaw.

To obtain the number of pyloric cæca the gut of each fish was taken out and preserved with a label attached bearing the same serial number as the fish.

The number of fin rays is given in each case as a total, but during the examination of the fin rays it was observed that an attempt might be 
made to analyse the fin rays in the dorsal and anal fins. The analysis of the vertebræ in this sample is the same as in the larger Samples III and IV (see pp. 80 and 82).

\section{Sample III.}

This was a sample of 550 fish examined from a catch of 22 cran, i.e. about 20,000 herrings. The fish were caught in herring drift nets by the steam-drifter Diadem, Lowestoft, near Bigbury Bay, with Start Point bearing about E. by N. 6 miles. The sample was taken at random from the catch, and consisted of fish of various sizes, but mostly in a condition approaching ripeness. Fish which were damaged were not investigated ; otherwise there was no selection.

In the circumstances under which the research was carried out it was possible to examine only 250 fish in measurements, weight and condition of gonad on the first day, that is within 12 hours of the landing of the fish. The fish not examined the first day were kept in ice, and were found to be in excellent preservation on the second day, when the remainder of the sample, namely 300 fish, was examined for measurements, weight and condition of gonad. The whole sample was examined within 35 hours of the landing of the fish, and a record of time was taken as the examination of each lot of 50 fish was completed. These records are given with those for Sample IV in tabular form on page 79.

\section{Sample IV.}

In this sample 525 fish out of a catch of 56 cran, i.e. about 50,500 herrings, were examined. The catch was taken by the steam-drifter G.M.V. 1062, Lowestoft, in herring drift nets near Bigbury Bay, between a region 8 miles W.S.W. of Start Point and a position about 3 miles S.S.W. of Start Point. The fish were caught during the night of January 5-6, 1915, and landed about 10 a.m., January 6 . Work was begun on the sample during the same morning, and 300 fish examined for weight, measurements and condition of gonad in the course of the day. The completion of the examination of the whole sample was effected within $33 \frac{1}{2}$ hours of the landing of the fish.

The fish were mostly in a condition almost ready for spawning, some few being spent. In this sample there were a good many damaged fish, and to obtain 525 fish from a sample of 600 it was necessary to reject about 40 to 50 fish, most of which were too badly damaged about the head to be measured. The damage to these specimens had undoubtedly chiefly occurred in unmeshing them. No selection of specimens occurred other than that of damaged ones.

The times at which successive batches were examined for weight, 
measurements and condition of gonad are shown with those for Sample III in the following table :-

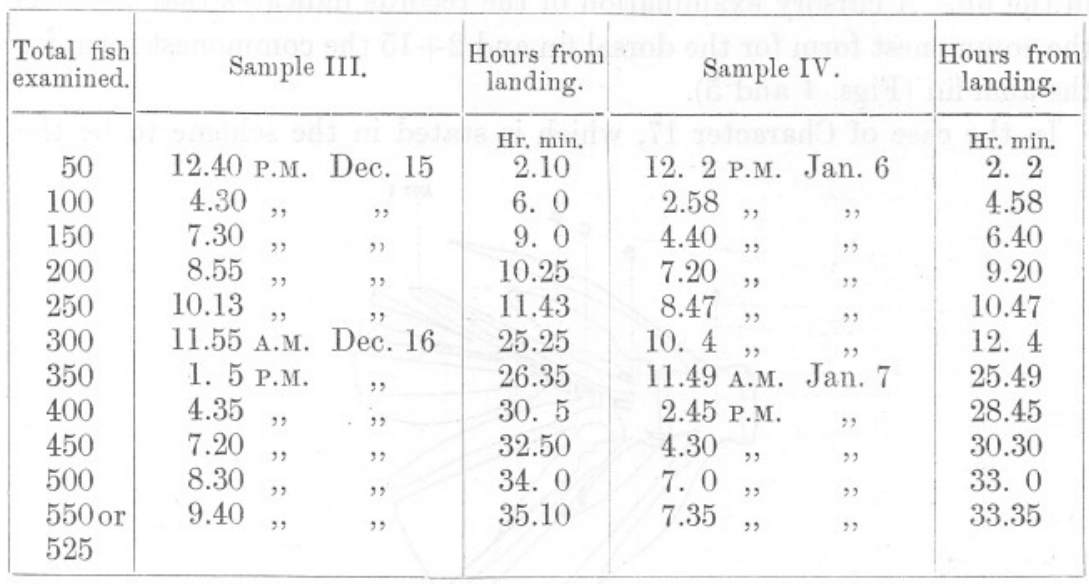

\section{EXPLANATION OF RECORDS OF CHARACTERS 1A AND 1в IN SAMPLES II AND III.}

In Sample III two characters in addition to those recommended by the Board were examined in a few fish. These characters are $1 a$ and $1 b$. Character $1 a$, as in Sample II, is the shortest distance between a tangent to the posterior border of the maxilla taken at right angles to the long axis of the fish, and a tangent to the tip of the lower jaw at right angles to the long axis of the fish. Character $1 b$ is the shortest distance between a tangent to the posterior border of the orbit taken at right angles to the long axis of the fish, and a similar tangent to the tip of the lower jaw.

It was found, however, that the taking of those measurements would decrease the number of fish examined within the shortest time recommended for the Characters 1 to 9 , hence it was decided at an early stage to discontinue to take the additional ones.

\section{EXPLANATION OF RECORDS OF CHARACTERS 13, 14, AND 17 IN SAMPLE III.}

With regard to Characters 13,14 and 17 on the sheets an attempt has been made in the case of the fins (13 and 14) to analyse them, and in the case of the vertebræ with hæmal arches to give additional information. The records for these characters are given in the general form of $a+b$.

In Sample III the dorsal and anal fins (13 and 14) were analysed in the following manner : in each case the fin-rays in the anterior portion of the fin equal to or less than two-thirds the height of the longest rays were 
counted separately from the fin-rays posterior to them; thus the records take the form of $a+b$, the sum of which gives the total number of rays in the fin. A cursory examination of the records indicates that $2+17$ is the commonest form for the dorsal fin and $2+15$ the commonest form for the anal fin (Figs. 4 and 5).

In the case of Character 17 , which is stated in the scheme to be the

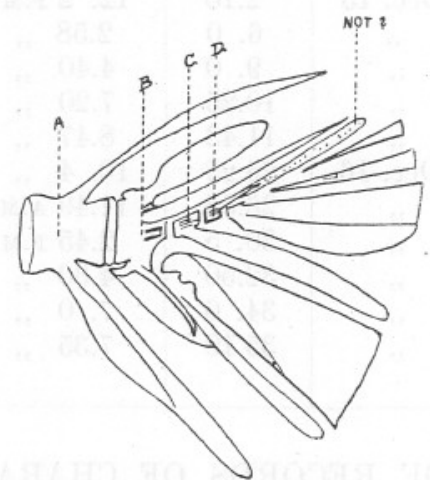

FIG. 2.-Diagram of Tail-bones of Herring, from Williamson. A is the last vertebra counted in the present work. B is regarded by Williamson as the last vertebra. Not $?=$ Notochord ?

"serial number of first vertebra having complete hæmal arch," the records have been made in the form of $(a+b)$ where $b=$ the total number of vertebræ with complete hæmal arch-not, however, counting the
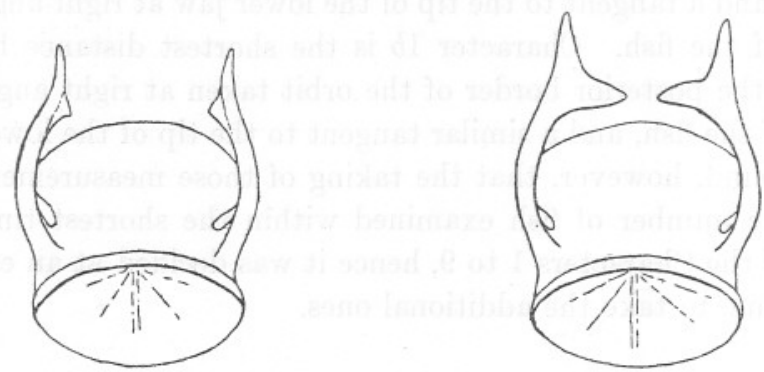

FIG. 3.-Vertebræ, showing incomplete but "well-developed" hæmal arch.

terminal vertebra-like ossicle regarded by Williamson as the "last vertebra" (see Fisheries, Scotland, Sci. Invest., 1914, I (April, 1914) Fig. 7, B, p. 21). Williamson's figure is here reproduced as Fig. 2.

In this character (17), $a=$ the number of vertebræ having an incomplete, but "well-developed " arch, and an arch was considered "well developed " if the hæmal processes were almost as large as those of the first complete arch, and if these processes possessed even the smallest trace of an internal cross-piece (see Figure 3). It should be mentioned that all inter- 
mediate stages are met with between a trace of an internal cross-piece and a complete arch. It is not improbable that in the living animal these arches are closed by a cartilaginous cross-piece. Cursory examination of the records indicates that in a majority of skeletons the sum of the number of vertebræ with complete hæmal arch and the number with welldeveloped (i.e. potentially complete?) arches is 33 ; or it might be said that the commonest number of vertebræ with potentialities for complete hæmal arches is 33 ; the greatest number of such vertebræ appears to be 35 .

\section{EXPLANATION OF CHARACTERS 13, 14, AND 17 IN SAMPLE IV.}

In Number IV Sample it was thought that more information could be obtained by analysing the dorsal and anal fin (Characters 13 and 14) in a slightly different way from that adopted in Sample III.

Thus in Sample IV all the anterior fin-rays of the dorsal fin which were distinctly shorter than the longest fin-ray were counted separately from the following and recorded in the general form of $a+b$, where $a$ is the

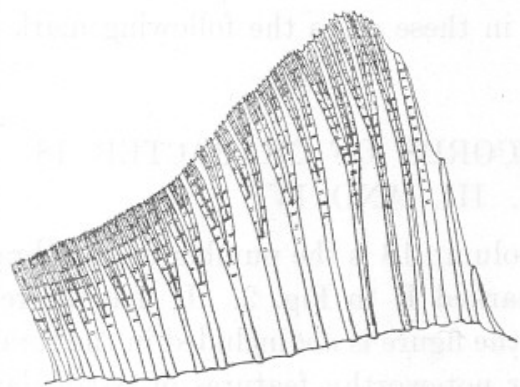

Frg. 4.-Dorsal Fin. In Sample III the above would be recorded as $2+17$; in Sample IV as $3+16$.

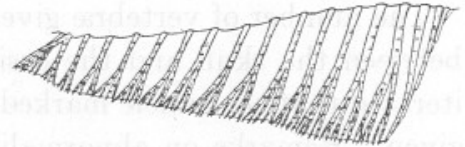

Fig. 5.-Anal Fin. In Sample III the above would be recorded as $2+15$; in Sample IV as $3+14$.

number of the smaller anterior rays and $b$ the number of rays posterior to these. In this way it is possible to reconstruct a fin to represent the commonest form which by cursory examination of the records is seen to be one having $3+16$ rays. A drawing of the type of ray is shown in Fig..

In the case of the anal fin (Character 14) the fin was analysed in the following manner : all the anterior fin-rays which were not subdivided at the tip or splayed out in any way were counted and recorded separately as " $a$ " from those in which the rays were splayed out-recorded as " $b$." The commonest form of fin is seen from the records to be one recorded as $3+14$. This type of fin is shown in Fig. 5 .

Character 17 in Sample IV is also recorded in a manner slightly different 
from that in Sample III. In this sample (IV) it was decided to include under "well-developed" open hæmal arches those in which the hæmal processes were relatively stout to those of the first closed arch, but which processes did not necessarily possess the trace of a cross-piece on their internal faces. This change of recording has resulted in only a slight difference in the records, but in a few cases the number of vertebræ

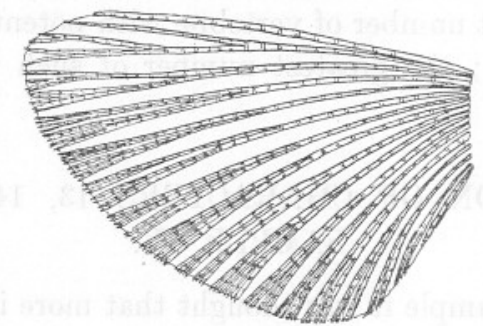

FIg, 6.-Pectoral Fin. 17 rays.

recorded in the " $a$ " category is higher than in corresponding skeletons in Sample III.

It should be mentioned that in some skeletons the hæmal processes of the vertebræ anterior to the first vertebra with complete arch were missing, having been cleaned away; in these cases the following mark $\uparrow$ is placed alongside the record.

\section{EXPLANATION OF THE RECORDS OF CHARACTER 18 IN SAMPLES II, III, AND IV.}

The number of vertebræ given in column 18 is the number of vertebræ between the skull and the ossicle marked B in Fig. 2. It may be reiterated that the ossicle marked B in the figure is not included in the total given. Remarks on abnormalities or noteworthy features of particular skeletons are connected by an asterisk to explanations in the Appendix to the Tables. It may be noted that in several skeletons two or more vertebræ have apparently become fused together; as, however, such fused vertebræ show uniformly only two articulations, they have in each case been counted as one vertebra, although it is most probable that most of these abnormal vertebræ are equivalent to two or more normal ones. Each case is discussed in the Appendix to the Tables.

\section{EXPLANATION OF RECORDS OF CHARACTERS 1 TO 9, 11, 12, 15, AND 16 IN SAMPLES III AND IV.}

Characters 11 and 12 call for little comment.

In counting the keeled scales between the pelvic fin and anus (11) the adjacent scales were cleaned well away before beginning to count. In 
this way the insertions of the keeled scales could be made out and their total number established with certainty. Practically no difficulty was experienced in counting the rays in the pectoral fin (12). It was noticed, however, that in fins with a large number of rays the increase in the number appeared to be accounted for by additions of small rays near the posterior border. No attempt was made to analyse this fin as in the case of the dorsal and anal fins, but it is possible that useful information might be obtained by attempting such an analysis.

The weight of the fish (15) was taken separately and to the nearest gram.

In recording the condition of the gonad (16) it was found necessary to use combinations of the numerals representing different stages, which require explaining.

In the records occur such combinations as IV-VI and VI-IV. A record such as IV-VI is put down to represent fish in which the gonad appeared to be about ripe, although it did not fill the body cavity entirely. These records, however, refer mostly to males, in which the approach to and incidence of ripeness of the gonad are not easy to differentiate. In the case of records such as VI-III or VI-IV, these indicate that the gonad is definitely ripe, but has become reduced by spawning (or compression in some cases) to the size in the stage indicated by the second numeral; thus VI-IV indicates gonad reduced to the size of half the volume of the abdominal cavity. The numeral VII was reserved for fish which were spent or practically spent. By distinguishing spawning fish in this way it is possible to correlate to some extent the weight with the size of the fish.

With regard to Characters 1 to 9 all measurements were taken with the instrument supplied by the Board of Agriculture and Fisheries. An attempt was made to measure Character 1 to the nearest $\cdot 2$ of a millimetre in Samples III and IV.

Character 2 was measured to the nearest $.5 \mathrm{~mm}$. in Sample III and to $.2 \mathrm{~mm}$. in Sample IV.

Characters 3 to 9 were measured to the nearest $.5 \mathrm{~mm}$. in both Samples III and IV.

\section{DEFINITION OF CHARACTER 7.}

Characters 1 to 9 are those recommended by the Board except No. 7 . Character 7 is defined in the Scheme as "from the end-board, etc. . . to the posterior edge of the hindmost scale." In preliminary investigations, however, it was found that posterior scales were either rubbed loose or missing in about one-third of the specimens examined. It was therefore 
decided to use some other fixed point of more constant position. The point chosen is in all probability the one shown in the figure of the Herring supplied by the Board. It is the point from which the perpendicular 7 arises, and marks the origin of the median caudal rays from the muscular part of the tail. The muscular part of the tail is covered by an epidermis of metallic appearance, and is in nearly all cases sharply marked off from what may be regarded as the tail fin proper where this kind of epidermis is absent. The caudal fin-rays are slightly embedded in the fleshy part of the tail. Thus the point chosen for measurement may be stated shortly to be the origin of the mid-caudal rays from the fleshy part of the tail.

The origin of these rays is, however, a concave line, as indeed is shown in the Board's figure, and the point actually measured is the line at right angles to the long axis of the fish which forms a tangent to the posterior border of the fleshy part of the tail. This line is apparently the same as the perpendicular No. 7 shown in the Board's figure.

There were only a few fish in which this point was at all difficult to determine and these were among Sample IV. It is of course well known that the posterior scales extend over the mid-caudal fin-rays.

\section{ACCURACY OF MEASUREMENTS.}

Before the large samples were examined a batch of 33 fish was examined twice, in order to obtain some determination of the error in measuring under the conditions in which the samples would be examined. The fish measured were not in good condition, so that it is probable that the errors observed in this case would be the maximum error, especially as more practice in measuring was obtained afterwards. In this experiment the average difference in the two sets of readings was less than $1 \mathrm{~mm}$. in all measurements except 4 and 6 , in which the average difference was $1 \cdot 3$ $\mathrm{mm}$. and $1.1 \mathrm{~mm}$. respectively. These 33 fish were examined in 47 and 45 minutes respectively, i.e. about the rate of 44 per hour, about the maximum rate for the large samples. After this experiment assistance was obtained in making measurements for Characters $3,4,5$, and 6 , and there can be no doubt that the accuracy of the measurements was thereby increased. In all measurements therefore it may be confidently stated that they are correct on the average to one millimetre, and in the case of 1 the average error is probably not more than $.5 \mathrm{~mm}$. It is believed that only isolated errors of measurement occur of as much as $3 \mathrm{~mm}$., but errors would increase in frequency towards zero.

It is, however, possible that occasional errors of observation may occur of as much as $5 \mathrm{~mm}$. where the $.5 \mathrm{~cm}$. line on the scale has been read as a 
whole $\mathrm{cm}$. division line, for one or two cases of this kind were actually observed in time to prevent this error. It is probably very difficult to exclude completely occasional lapses of this nature in examining large numbers of fish at the high rate of speed required.

It is unfortunate in some respects that the experimental sample mentioned above was not a fresh sample exactly comparable with a research sample, and it would probably be better in future work to re-examine a batch of the research sample in order to determine the error of measurement.

An experiment was carried out to determine how accurately the instrument would measure. A number of slips of paper (30) were ruled with lines parallel to one end, which was placed against and was parallel with the " end-board" of the instrument. The points measured on the ruled lines were similar to those measured on herrings, so that in measuring them it was necessary to move the instrument about in approximately the same way as when measuring the research herrings. The slips of paper represented in fact paper herrings. These 30 slips were measured twice at a rate greater than the maximum rate at which the research samples of herrings were examined. Characters 1 and 2 were read to $\cdot 2 \mathrm{~mm}$., and Characters 3 to 9 to the nearest $.5 \mathrm{~mm}$., just as in the research sample. The average difference between the first and second measurements of Characters 1 and 2 was less than $\cdot 1 \mathrm{~mm}$., and in only 4 cases was the difference as much as $\cdot 3 \mathrm{~mm}$. The average difference between all the measurements of Characters 1 and 2 and the actual distance-as measured by a $15-\mathrm{cm}$. ivory rule divided to fifths of a millimetre - was also less than $\cdot 1 \mathrm{~mm}$., and in only 5 cases were there differences of $\cdot 3 \mathrm{~mm}$.

The average difference between first and second measurements of Characters 3 to 9 was less than $\cdot 1 \mathrm{~mm}$., and in only one case was the difference more than $.5 \mathrm{~mm}$. The difference was exactly $.5 \mathrm{~mm}$. in 30 cases, and in 159 pairs of measurements the results were exactly the same. In all the measurements of Characters 3 to 9 the average difference from the actual distance measured was about $\cdot 13 \mathrm{~mm}$.

In a large number of measurements, however, it is considered that the instrument may be taken as reading accurately on the average, since the plus and minus variations would tend to balance each other, although ranging between plus and minus the maximum error mentioned above. The average algebraical error of all the measurements taken in the paper-herring sample mentioned above was less than $+.03 \mathrm{~mm}$. in Characters 1 and 2 , and $+\cdot 04 \mathrm{~mm}$. in Characters 3 to 9 . 


\section{REMARKS ON ADDITIONAL CHARACTERS TO THOSE RECOMMENDED BY THE BOARD.}

In Sample II two additional characters to those recommended by the Board were examined, namely, the relation of the posterior border of the maxilla to the orbit, and the number of pyloric cæca. In the large sample it was found impracticable to examine the former character in addition to the Board's characters, owing to the exigencies of time, but the pyloric cæca of all the fish in Samples III and IV have been preserved with their proper number, and can be examined and recorded at leisure.

The examination of these characters is considered of equal importance to those recommended by the Board, since they are characters in which Clupea harengus differs from allied species. 
HAUL.

Table I. Herring Race Investigations.

SAMPLE I.

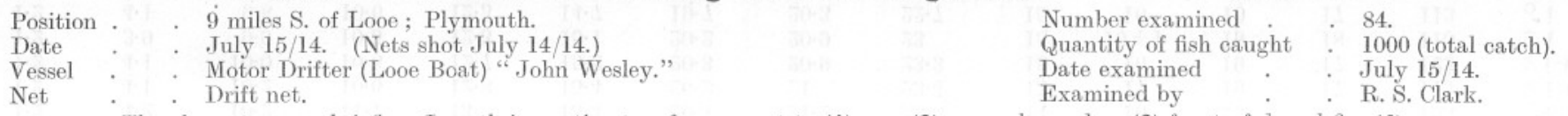

The characters are briefly: Length in centimetres from snout to (1) eye, (2) operculum edge, (3) front of dorsal fin, (4) pelvic, (5) back of dorsal, (6) front of anal, (7) root of tail, (8) end of mid-caudal, (9) end of longest caudal ray. Number of (11) keeled scales (pelvics to anus), (12) rays in pectoral, (13) rays in dorsal, (14) rays in anal, (15) weight in grams, (16)

\begin{tabular}{|c|c|c|c|c|c|c|c|c|c|c|c|c|c|c|c|c|}
\hline No. of & & 2 & $\begin{array}{c}3 \\
3\end{array}$ & & & & & & & & & & & & & \\
\hline fish. & 1 & 2 & 3 & 4 & 5 & 6 & 7 & 8 & 9 & 11 & 12 & 13 & 14 & 15 & 16 & \\
\hline 1 & $1 \cdot 3$ & $4 \cdot 2$ & $10 \cdot 2$ & $10 \cdot 5$ & $12 \cdot 5$ & $14 \cdot 8$ & $19 \cdot 7$ & $20 \cdot 3$ & $22 \cdot 6$ & 15 & 16 & 19 & 19 & 107 & 우 $\mathrm{i}$ & \\
\hline 2 & $1 \cdot 4$ & $4 \cdot 4$ & $10 \cdot 9$ & $11 \cdot 1$ & $13 \cdot 2$ & $15 \cdot 8$ & $20 \cdot 9$ & $21 \cdot 7$ & 24 & 14 & 16 & 18 & 18 & 123 & $\hat{\mathrm{o}} \mathrm{i}-\mathrm{ii}$ & \\
\hline 3 & $1 \cdot 3$ & $4 \cdot 1$ & 10 & $10 \cdot 4$ & $12 \cdot 6$ & 15 & $19 \cdot 7$ & $20 \cdot 5$ & $22 \cdot 5$ & 14 & 17 & 19 & 18 & 104 & i $\mathrm{i}$ & \\
\hline 4 & $1 \cdot 2$ & $3 \cdot 7$ & $9 \cdot 6$ & $10 \cdot 1$ & $11 \cdot 8$ & 14.9 & $19 \cdot 4$ & $20 \cdot 3$ & $22 \cdot 3$ & 15 & $18 ?$ & 18 & 17 & 91 & i $\mathrm{i}$ & 幽 \\
\hline 5 & $1 \cdot 3$ & $4 \cdot 3$ & $10 \cdot 1$ & $10 \cdot 6$ & $12 \cdot 6$ & $15 \cdot 1$ & $20 \cdot 3$ & $21 \cdot 1$ & $23 \cdot 3$ & 15 & 16 & 20 & 17 & 129 & i-ii & 望 \\
\hline 6 & $1 \cdot 4$ & $4 \cdot 5$ & $11 \cdot 6$ & $12 \cdot 2$ & $14 \cdot 3$ & $17 \cdot 3$ & $22 \cdot 7$ & $23 \cdot 8$ & $26 \cdot 1$ & 15 & 16 & 20 & 18 & 151 & 우 & 或 \\
\hline 7 & $1 \cdot 3$ & $4 \cdot 2$ & $10 \cdot 3$ & $10 \cdot 4$ & 12.7 & $15 \cdot 5$ & $20 \cdot 3$ & $21 \cdot 3$ & $23 \cdot 6$ & 14 & 17 & 19 & 16 & 120 & i & $=$ \\
\hline 8 & $1 \cdot 3$ & $4 \cdot 3$ & $11 \cdot 1$ & $11 \cdot 6$ & $13 \cdot 9$ & $16 \cdot 7$ & 22 & $22 \cdot 8$ & $25 \cdot 2$ & 15 & 17 & 19 & 17 & 146 & i $\mathrm{i}$ & \\
\hline 9 & $1 \cdot 3$ & $4 \cdot 2$ & $10 \cdot 4$ & 11 & $12 \cdot 8$ & $15 \cdot 6$ & $20 \cdot 1$ & $21 \cdot 3$ & $23 \cdot 5$ & 15 & 17 & 20 & 18 & 112 & i & 0 \\
\hline 10 & $1 \cdot 2$ & 4 & $9 \cdot 8$ & $10 \cdot 4$ & $12 \cdot 3$ & $14 \cdot 5$ & $19 \cdot 6$ & $20 \cdot 3$ & $22 \cdot 3$ & 14 & 16 & 19 & 17 & 100 & $\mathrm{o}^{*} \mathrm{i}$ & \\
\hline 11 & $1 \cdot 3$ & 4 & $10 \cdot 3$ & $10 \cdot 9$ & $12 \cdot 8$ & $15 \cdot 4$ & $20 \cdot 4$ & $21 \cdot 4$ & $23 \cdot 5$ & 15 & 16 & 19 & 18 & 118 & $\mathrm{o}^{1} \mathrm{i}$ & \\
\hline 12 & $1 \cdot 2$ & $4 \cdot 3$ & $10 \cdot 6$ & $11 \cdot 2$ & $13 \cdot 2$ & $15 \cdot 8$ & $20 \cdot 8$ & $21 \cdot 8$ & $24 \cdot 2$ & 15 & 17 & 19 & 18 & 121 & i & \\
\hline 13 & $1 \cdot 2$ & 4 & $10 \cdot 3$ & $10 \cdot 5$ & $12 \cdot 8$ & $15 \cdot 4$ & 20 & 21 & $23 \cdot 1$ & 15 & 16 & 19 & 18 & 112 & i & \\
\hline 14 & $1 \cdot 2$ & 4 & $10 \cdot 1$ & $10 \cdot 3$ & $12 \cdot 4$ & $15 \cdot 0$ & $19 \cdot 6$ & $20 \cdot 2$ & $22 \cdot 2$ & 15 & 15 & 19 & 17 & 101 & ô $\mathrm{i}-\mathrm{ii}$ & 4 \\
\hline 15 & $1 \cdot 2$ & $4 \cdot 1$ & $10 \cdot 3$ & $10 \cdot 6$ & $12 \cdot 7$ & $15 \cdot 1$ & $20 \cdot 1$ & $20 \cdot 7$ & $23 \cdot 1$ & 15 & 16 & 19 & 17 & 116 & 우 $\mathrm{i}-\mathrm{ii}$ & 정 \\
\hline 16 & $1 \cdot 2$ & $4 \cdot 1$ & $10 \cdot 1$ & $10 \cdot 8$ & $12 \cdot 9$ & $15 \cdot 3$ & $20 \cdot 2$ & $21 \cdot 2$ & $23 \cdot 5$ & 14 & 15 & 19 & 19 & 122 & ㅇ $\mathrm{i}$ & \\
\hline 17 & $1 \cdot 2$ & $3 \cdot 9$ & $9 \cdot 7$ & $9 \cdot 9$ & 12 & $14 \cdot 5$ & $18 \cdot 8$ & $19 \cdot 6$ & $21 \cdot 6$ & 15 & 17 & 19 & 18 & 82 & 우 & 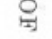 \\
\hline 18 & $1 \cdot 3$ & $4 \cdot 3$ & $10 \cdot 5$ & $10 \cdot 8$ & $13 \cdot 4$ & $15 \cdot 5$ & $20 \cdot 9$ & $21 \cdot 6$ & $24 \cdot 1$ & 15 & 17 & 19 & 18 & 126 & ô $\mathrm{i}-\mathrm{ii}$ & \\
\hline 19 & $1 \cdot 3$ & $4 \cdot 2$ & $10 \cdot 5$ & 11 & $12 \cdot 9$ & $16 \cdot 1$ & 21 & $21 \cdot 8$ & $24 \cdot 1$ & 15 & 17 & 18 & 18 & 126 & o i-ii & \\
\hline 20 & $1 \cdot 2$ & $3 \cdot 9$ & $9 \cdot 4$ & $10 \cdot 1$ & $11 \cdot 9$ & $14 \cdot 5$ & $18 \cdot 8$ & $19 \cdot 8$ & 22 & 15 & 16 & 19 & 18 & 90 & i & 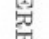 \\
\hline 21 & $1 \cdot 3$ & $4 \cdot 2$ & $10 \cdot 1$ & $10 \cdot 7$ & $12 \cdot 6$ & 15 & $19 \cdot 8$ & $20 \cdot 8$ & $23 \cdot 1$ & 14 & 17 & 19 & 19 & 118 & 우 $\mathrm{i}-\mathrm{ii}$ & 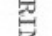 \\
\hline 22 & $1 \cdot 3$ & $4 \cdot 1$ & $10 \cdot 2$ & $10 \cdot 7$ & $12 \cdot 7$ & $15 \cdot 3$ & 20 & $20 \cdot 7$ & 23 & 16 & 17 & 20 & 17 & 100 & 우 $\mathrm{i}$ & 7 \\
\hline 23 & $1 \cdot 3$ & $4 \cdot 5$ & $11 \cdot 1$ & $11 \cdot 6$ & $13 \cdot 7$ & $16 \cdot 7$ & $21 \cdot 5$ & $22 \cdot 6$ & $25 \cdot 1$ & 15 & 16 & 19 & 19 & 138 & 우 $\mathrm{i}-\mathrm{ii}$ & is \\
\hline 24 & $1 \cdot 2$ & 4 & $9 \cdot 9$ & $10 \cdot 3$ & $12 \cdot 3$ & $14 \cdot 9$ & $19 \cdot 7$ & $20 \cdot 4$ & $22 \cdot 7$ & 14 & 17 & 19 & 18 & 111 & i & \\
\hline 25 & $1 \cdot 2$ & $4 \cdot 2$ & $9 \cdot 8$ & $10 \cdot 7$ & 12.5 & $15 \cdot 1$ & $20 \cdot 1$ & 21 & $22 \cdot 3$ & 14 & 17 & 19 & 18 & 114 & $0 \mathrm{i}$ & \\
\hline 26 & $1 \cdot 2$ & $3 \cdot 8$ & 10 & $10 \cdot 6$ & $12 \cdot 3$ & 15 & $19 \cdot 8$ & $20 \cdot 8$ & $22 \cdot 6$ & 15 & 15 & 17 & 16 & 102 & $o^{1} \mathrm{i}$ & \\
\hline 27 & $1 \cdot 2$ & $4 \cdot 2$ & $10 \cdot 2$ & $10 \cdot 8$ & $12 \cdot 8$ & $15 \cdot 9$ & $20 \cdot 6$ & $21 \cdot 5$ & $23 \cdot 8$ & 14 & 16 & 19 & 17 & 128 & i-ii & \\
\hline 28 & $1 \cdot 3$ & $4 \cdot 3$ & $10 \cdot 3$ & 11 & $12 \cdot 7$ & $15 \cdot 4$ & 20 & $21 \cdot 0$ & $23 \cdot 3$ & 14 & 16 & 18 & 16 & 121 & ㅇi & \\
\hline 29 & $1 \cdot 2$ & $4 \cdot 1$ & $9 \cdot 8$ & $10 \cdot 5$ & $12 \cdot 4$ & 15 & $19 \cdot 9$ & $20 \cdot 6$ & $22 \cdot 7$ & 15 & 17 & 18 & 18 & 107 & o i-ii & \\
\hline 30 & $1 \cdot 2$ & $4 \cdot 1$ & 10 & $10 \cdot 3$ & $12 \cdot 4$ & 15 & $19 \cdot 7$ & $20 \cdot 7$ & 23 & 15 & 16 & 19 & 17 & 100 & ㅇi-ii & \\
\hline 31 & $1 \cdot 2$ & $4 \cdot 2$ & $10 \cdot 1$ & $10 \cdot 6$ & $12 \cdot 7$ & $15 \cdot 1$ & $19 \cdot 9$ & $20 \cdot 7$ & $22 \cdot 8$ & 14 & 17 & 19 & 18 & 114 & t $\mathrm{i}-\mathrm{ii}$ & \\
\hline 32 & $1 \cdot 1$ & 4 & $9 \cdot 6$ & $10 \cdot 4$ & $12 \cdot 2$ & $14 \cdot 3$ & $19 \cdot 3$ & 20 & $22 \cdot 2$ & 15 & 17 & 19 & 18 & 114 & $\mathrm{o}^{-1} \mathrm{i}$ & $\infty$ \\
\hline 33 & $1 \cdot 1$ & 4 & $10 \cdot 1$ & $10 \cdot 3$ & $12 \cdot 4$ & $14 \cdot 7$ & $19 \cdot 4$ & $20 \cdot 1$ & $22 \cdot 4$ & 14 & 17 & 19 & 19 & 105 & $\mathrm{o}^{*} \mathrm{i}$ & \\
\hline 34 & $1 \cdot 2$ & $4 \cdot 1$ & $10 \cdot 1$ & $10 \cdot 8$ & $12 \cdot 6$ & $15 \cdot 1$ & $20 \cdot 2$ & $20 \cdot 9$ & $23 \cdot 1$ & 15 & 17 & 19 & 18 & 113 & $\widehat{o} \mathrm{i}-\mathrm{ii}$ & \\
\hline 35 & $1 \cdot 2$ & $4 \cdot 2$ & $10 \cdot 3$ & $10 \cdot 7$ & $12 \cdot 7$ & $15 \cdot 2$ & $20 \cdot 2$ & $21 \cdot 1$ & $23 \cdot 3$ & & & & & & & \\
\hline 36 & $1 \cdot 2$ & 4 & $9 \cdot 3$ & 10 & $11 \cdot 9$ & $14 \cdot 1$ & $18 \cdot 7$ & $19 \cdot 4$ & $21 \cdot 7$ & 15 & 17 & 19 & 18 & 92 & 오 $\mathrm{i}-\mathrm{ii}$ & \\
\hline 37 & $1 \cdot 2$ & 4 & $9 \cdot 8$ & $10 \cdot 6$ & $12 \cdot 6$ & $15 \cdot 1$ & $19 \cdot 7$ & $20 \cdot 6$ & $22 \cdot 9$ & 15 & 16 & 19 & 17 & 114 & $\widehat{o} \mathrm{i}-\mathrm{ii}$ & \\
\hline
\end{tabular}




$\begin{array}{cc}2 & 3 \\ 4 \cdot 3 & 11 \cdot 3\end{array}$

45

$\begin{array}{cc}6 & 7 \\ 17 \cdot 3 & 22 \cdot 2\end{array}$

$23 \cdot 1$

(1) $11 \quad 12 \quad 13$

\begin{tabular}{|c|c|c|}
\hline 14 & 15 & 16 \\
\hline 18 & 154 & $\hat{\delta} \mathrm{ii}$ \\
\hline 17 & 140 & i-ii \\
\hline 18 & 112 & i \\
\hline 19 & 113 & $\dot{+} \mathrm{i}-\mathrm{ii}$ \\
\hline $16 ?$ & 115 & $\widehat{o} \mathrm{i}$ \\
\hline
\end{tabular}

$\begin{array}{lllll}10 \cdot 1 & 10 \cdot 6 & 12 \cdot 8 & 15 \cdot 1 & 20 \cdot 2 \\ 9 \cdot 9 & 10 \cdot 6 & 12 \cdot 4 & 15 \cdot 2 & 20 \cdot 2\end{array}$

$22 \cdot 8$

14

17

$10 \cdot 1$
4 $\quad 9 \cdot 9$

$15 \cdot 2-20 \cdot 2$

$21 \cdot 5$

$23 \cdot 1$

14

$9 \cdot 9$
10

$10 \cdot 5$

$12 \cdot 4$

$15 \cdot 1$

$\begin{array}{ll}19 \cdot 2 & 20 \cdot 2 \\ 19 \cdot 4 & 20 \cdot 3\end{array}$

$\begin{array}{lll}10 \cdot 3 & 10 \cdot 7 & 12 \cdot 8\end{array}$

15

$20 \cdot 1$

$21 \cdot 1$

$23 \cdot 3$

$\begin{array}{lllll}10 \cdot 8 & 11 \cdot 3 & 13.5 & 16 \cdot 3 & 21.5 \\ 10 \cdot 4 & 11.2 & 13 & 15.5 & 20.7\end{array}$

$22 \cdot 3$

$10 \cdot 4$

$11 \cdot 2$

$13 \cdot 5$

$15 \cdot 8$

$21 \cdot 3$

$21 \cdot 5$

$23 \cdot 4$

$24 \cdot 9$

$22 \cdot 1 \quad 24 \cdot 4$

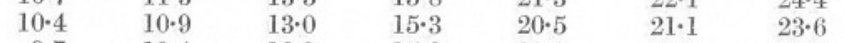

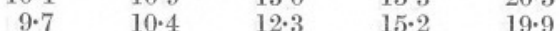

$20 \cdot 6$

$23 \cdot 6$
$22 \cdot 9$

$\begin{array}{rr}9 \cdot 7 & 10 \cdot 4 \\ 10 \cdot 4 & 11 \cdot 3\end{array}$

$10 \cdot 5$

$11 \cdot 3$

$12 \cdot 9$

$16 \cdot 2$

$19 \cdot 9$
$20 \cdot 6$

$21 \cdot 6$

$10 \cdot 9 \quad 15 \cdot 5 \cdot 21 \cdot 2$

$24 \cdot 0$

$23 \cdot 5$

$20 \cdot 3 \quad 23 \cdot 2$

$\begin{array}{rr}9 \cdot 7 & 10 \cdot 4 \\ 10 \cdot 7 & 11 \cdot 4\end{array}$

$12 \cdot 1$

$14 \cdot 8$

$19 \cdot 6$

$22 \cdot 1$

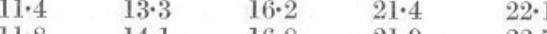

$11 \cdot 8-14 \cdot 1-16 \cdot 8$

$22 \cdot 7$

$24 \cdot 5$

$11 \cdot 4$

$10 \cdot 6$

$\begin{array}{ll}16 \cdot 8 & 21 \cdot 9 \\ 14 \cdot 5 & 19 \cdot 3\end{array}$

$20 \cdot 2$

$11 \cdot 2$

$13 \cdot 4$

16

$21 \cdot 5$

$22 \cdot 3$

20

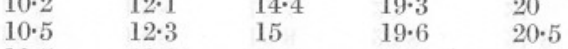

$\begin{array}{llll}10 \cdot 5 & 12 \cdot 2 & 15 \cdot 3 & 19 \cdot 9\end{array}$

$20 \cdot 6$
$20 \cdot 4$
20

20

$21 \cdot 4$

$19 \cdot 7$
$20 \cdot 5$

$\begin{array}{ll}20 \cdot 5 & 22 \cdot 8 \\ 19 \cdot 4 & 21 \cdot 5\end{array}$

21
$19 \cdot 9$

$10 \cdot 1$

$9 \cdot 5$

$15 \quad 19 \cdot 6$

$10 \cdot 9 \quad 12 \cdot 6 \quad 14 \cdot 4 \quad 19 \cdot 3$

$\begin{array}{llll}10 \cdot 2 & 11 \cdot 8 & 15 \cdot 2 & 20 \cdot 2\end{array}$

14
15
14
15
15
14
14
15
14
15
14
14
15
14
15
15
15
14
15
15
14
16
14
15
13
14
15
14
14
15
15
15
15
14
14
14
14
15
15
15
15
15


Table II. Herring Race Investigations.

HAUt.

Position . . Cawsand Bay (condition of fish : very good).
Date $\quad$. Early morning Dec. 9/14. Landed morning of Dec. 9/14.

SAMPLE II.

- Early morning Dec. 9/14. Landed morning of Dec, 9/14.

Number examined

$\begin{array}{ll}\text { Quantity of fish caught } & 250-300 . \\ \text { Date examined } & \text { Dec. } 9 / 1\end{array}$

Not known.

Dec. $9 / 14$.

Net.

Examined by .

J. H. Orton.

The characters are briefly: Length in centimetres from snout to (1) eye, (2) operculum edge, (3) front of dorsal fin, (4) pelvic, (5) back of dorsal, (6) frant of anal, (7) root of tail, (8) end of mid-caudal, (9) end of longest caudal ray, Number of

sex and maturity, (17) vertebre with hæmal arch, (18) total vertebræ.

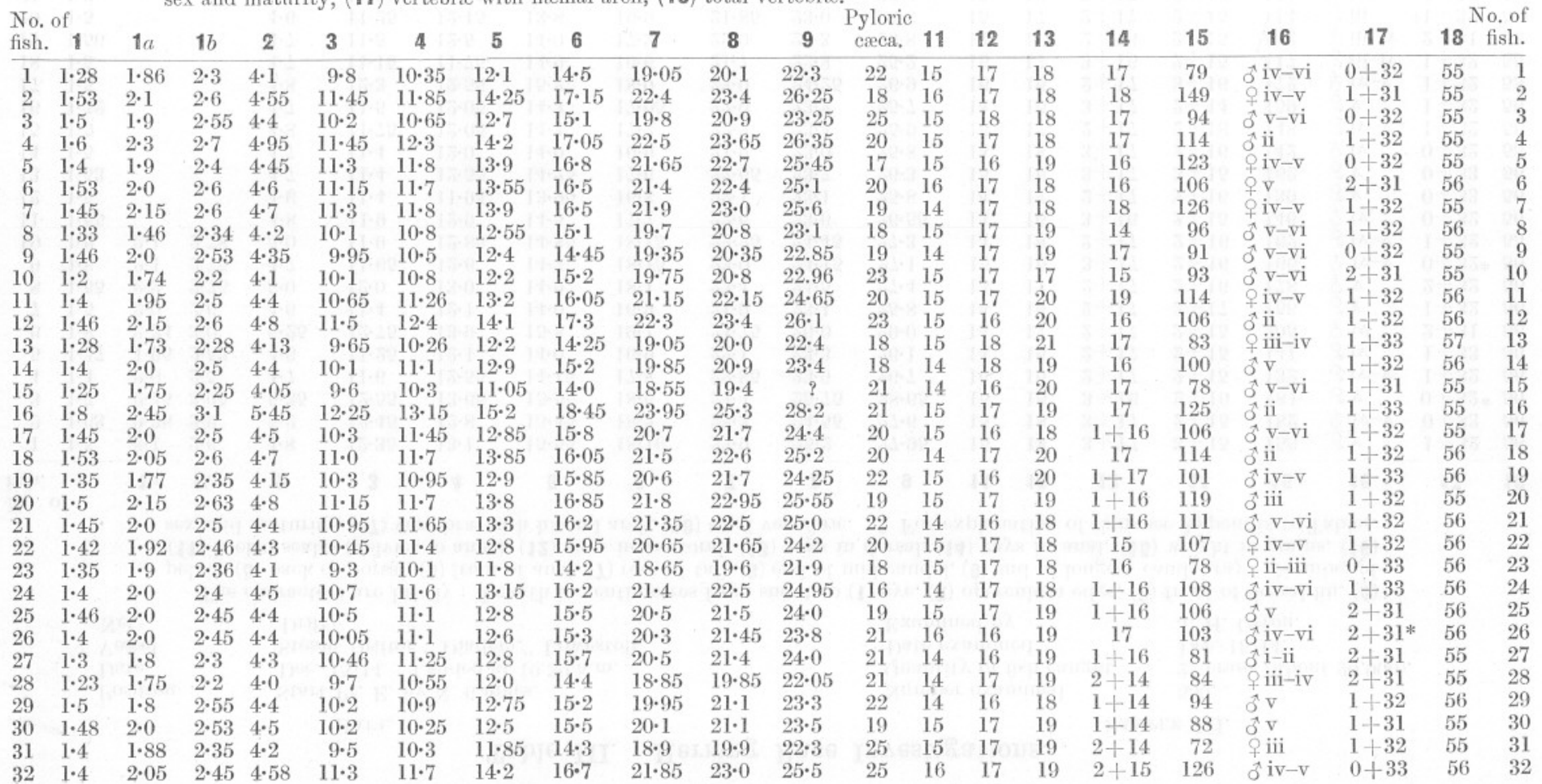


Table III. Herring Race Investigations.

HAUL.

Number examined . . 550 .

Position . . Start Pt. E. by N. 6 miles.

550.

Date examined $\quad$ Dec. $15 / 14$

Examined by

Vessel . . Steam Drifter "Diadem," Lowestoft.

Drift.

The characters are briefly: Length in centimetres from snout to (1) eye, (2) operculum edge, (3) front of dorsal fin, (4) pelvic, (5) back of dorsal, (6) front of anal, (7) root of tail, (8) end of mid-caudal, (9) end of longest caudal ray. Number of (11) keeled scales (pelvics to anus), (12) rays in pectoral, (13) rays in dorsal, (14) rays in anal, (15) weight in grams, (16)

\begin{tabular}{|c|c|c|c|c|c|c|c|c|c|c|c|c|c|c|c|c|c|c|c|}
\hline fish. & 1 & $1 a$ & $1 b$ & 2 & 3 & 4 & 5 & 6 & 7 & 8 & 9 & 11 & 12 & 13 & 14 & 15 & 16 & 17 & \\
\hline 1 & $1 \cdot 5$ & $2 \cdot 1$ & $2 \cdot 6$ & $4 \cdot 8$ & $12 \cdot 35$ & $13 \cdot 1$ & $15 \cdot 55$ & $18 \cdot 16$ & $23 \cdot 9$ & $25 \cdot 2$ & $27 \cdot 95$ & 15 & 18 & $3+17$ & $3+15$ & 156 & $\mathrm{O}^{\hat{T}} \mathrm{v}$ & $1+32$ & \\
\hline 2 & $1 \cdot 63$ & $2 \cdot 36$ & $2 \cdot 9$ & $5 \cdot 2$ & $2 \cdot 45$ & $12 \cdot 8$ & $15 \cdot 45$ & $18 \cdot 2$ & $23 \cdot 3$ & $24 \cdot 55$ & $27 \cdot 6$ & 15 & 18 & $3+17$ & & 182 & $+\mathrm{iv}-\mathrm{v}$ & $0+33$ & \\
\hline 3 & 1.7 & $2 \cdot 45$ & $3 \cdot 05$ & $5 \cdot 35$ & 12.55 & $13 \cdot 65$ & $15 \cdot 55$ & $18 \cdot 6$ & $24 \cdot 4$ & $25 \cdot 75$ & $28 \cdot 65$ & 15 & 16 & $3+16$ & $2+16$ & 181 & $\hat{o v}$ & $0+32 *$ & \\
\hline 4 & $1 \cdot 4$ & $2 \cdot 0$ & $2 \cdot 5$ & $4 \cdot 7$ & $11 \cdot 6$ & $12 \cdot 55$ & $14 \cdot 15$ & $17 \cdot 8$ & $22 \cdot 85$ & $23 \cdot 9$ & $26 \cdot 7$ & 16 & 16 & $2+17$ & $2+15$ & 132 & ô $\mathrm{iv}-\mathrm{v}$ & $1+32$ & \\
\hline 5 & $1 \cdot 47$ & $1 \cdot 95$ & $2 \cdot 53$ & $4 \cdot 6$ & $11 \cdot 25$ & $12 \cdot 1$ & $14 \cdot 0$ & $16 \cdot 9$ & $22 \cdot 1$ & $23 \cdot 3$ & $26 \cdot 1$ & 14 & 16 & $2+17$ & $2+15$ & 141 & ô iv & $1+33$ & \\
\hline 6 & $1 \cdot 8$ & $2 \cdot 34$ & $3 \cdot 0$ & $5 \cdot 25$ & $12 \cdot 75$ & $13 \cdot 9$ & $15 \cdot 8$ & $19 \cdot 1$ & $24 \cdot 75$ & $26 \cdot 0$ & $29 \cdot 0$ & 14 & 17 & $2+17$ & $2+15$ & 205 & 우 iv & $2+31$ & \\
\hline 7 & 1.5 & $2 \cdot 0$ & $2 \cdot 6$ & $4 \cdot 6$ & $11 \cdot 4$ & $12 \cdot 1$ & $14 \cdot 0$ & $16 \cdot 9$ & $21 \cdot 9$ & $23 \cdot 1$ & $25 \cdot 8$ & 14 & 16 & $2+17$ & $2+17$ & 156 & $\hat{o v}$ & $1+32$ & \\
\hline 8 & 1.55 & $2 \cdot 2$ & $2 \cdot 75$ & $5 \cdot 0$ & $12 \cdot 0$ & $13 \cdot 0$ & & $18 \cdot 1$ & $23 \cdot 4$ & $24 \cdot 5$ & $27 \cdot 4$ & 15 & 17 & $2+17$ & $2+16$ & 178 & o v & $2+32$ & \\
\hline 9 & $1 \cdot 6$ & $2 \cdot 1$ & $2 \cdot 75$ & $4 \cdot 7$ & $11 \cdot 65$ & $12 \cdot 6$ & 14 & $18 \cdot 15$ & $23 \cdot 0$ & 24 & $27 \cdot 1$ & $1:$ & 16 & $3+$ & $2+16$ & 166 & iv-v & $0+32^{*}$ & \\
\hline 10 & 1.6 & $2 \cdot 1$ & 2.78 & $5 \cdot 0$ & $11 \cdot 9$ & $12 \cdot 85$ & 14 . & $18 \cdot 15$ & $23 \cdot 25$ & $24 \cdot 45$ & $27 \cdot 3$ & 14 & 18 & $2+17$ & $2+16$ & 162 & ô iv-v & $1+32$ & \\
\hline 11 & 1.55 & & & $4 \cdot 8$ & $11 \cdot 9$ & $12 \cdot 9$ & $14 \cdot 5$ & $17 \cdot 7$ & $22 \cdot 6$ & $23 \cdot 6$ & $26 \cdot 55$ & 14 & 16 & $3+16$ & $2+15$ & 146 & ơ iv & $0+32$ & \\
\hline 12 & 1.5 & & & $4 \cdot 6$ & $11 \cdot 4$ & 11.95 & 13 & $16 \cdot 8$ & & & $25 \cdot 8$ & 14 & 17 & $2+$ & 6 & 13 & $\mathrm{O} \mathbf{v}$ & & \\
\hline 13 & 1.53 & & & 4.7 & $11 \cdot 4$ & .55 & $14 \cdot 25$ & 17. & $22 \cdot 65$ & 23 & $26 \cdot$ & 1. & 16 & $3+$ & $3+$ & 16 & $\hat{\sigma} \mathrm{v}$ & $0+33$ & \\
\hline 14 & 1.5 & & & 4.7 & $11 \cdot 4$ & $12 \cdot 0$ & $14 \cdot 0$ & 16 . & $21 \cdot 9$ & 23 & $25 \cdot 8$ & 14 & 18 & $3+$ & $2+$ & 14 & iv & $0+32$ & \\
\hline 15 & 1.5 & & & $4 \cdot 8$ & $11 \cdot 75$ & 12.05 & 14 & 17 & 22 & 23 & 25 & 1. & 17 & $2+$ & $2+$ & 148 & $\hat{o} \mathrm{iv}-\mathrm{v}$ & $1+$ & \\
\hline 16 & 1.52 & & & $4 \cdot 7$ & 11 & 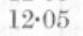 & & $17 \cdot 05$ & 22 & 23 & $25 \cdot 7$ & 14 & 16 & $3+$ & $2+$ & 159 & $\mathrm{o}^{2} \mathrm{v}$ & $1+32$ & \\
\hline 17 & 1.5 & & & $4 \cdot 8$ & $12 \cdot 3$ & 55 & 15 & $18 \cdot 0$ & $23 \cdot 0$ & $24 \cdot 25$ & $26 \cdot 9$ & 16 & $1 f$ & $2+17$ & $3+$ & 172 & iv-v & $1+32$ & \\
\hline 18 & 1.5 & & & $4 \cdot 7$ & $11 \cdot 15$ & $11 \cdot 75$ & $14 \cdot 9$ & $16 \cdot 6$ & $21 \cdot 7$ & $22 \cdot 8$ & $25 \cdot 2$ & 18 & 17 & $3+$ & & 117 & ô iii-iv & $1+32$ & \\
\hline 19 & 1.56 & & & $4 \cdot 7$ & $11 \cdot 5$ & & 14 & 17 & 2 & 23 & $25 \cdot 8$ & 14 & 1 & $2+$ & & 152 & 우 iii-iv & $2+31$ & \\
\hline 20 & 1.5 & & & $4 \cdot 6$ & $11 \cdot 25$ & & & 16 & $21 \cdot 85$ & $23 \cdot 0$ & $25 \cdot 6$ & 15 & 17 & $2+17$ & $2+15$ & 114 & o iii & $+1+32$ & \\
\hline 21 & $1 \cdot 6$ & & & $4 \cdot 9$ & 11.95 & $12 \cdot 35$ & $14 \cdot 85$ & $17 \cdot 4$ & $22 \cdot 6$ & $23 \cdot 7$ & $26 \cdot 6$ & 13 & 16 & $3+17$ & $2+16$ & 152 & $\mathrm{iv}-\mathrm{v}$ & & \\
\hline 22 & $1 \cdot 46$ & & & $4 \cdot 6$ & $12 \cdot 15$ & 10.65 & $14 \cdot 9$ & $17 \cdot 45$ & $22 \cdot 8$ & $24 \cdot 0$ & $26 \cdot 45$ & 13 & 17 & $2+17$ & 3 & 18 & & & \\
\hline 23 & 1.47 & & & $4 \cdot$ & $11 \cdot 2$ & 12. & & $17 \cdot 3$ & 22 & 23 & $25 \cdot 8$ & 15 & 16 & $3+17$ & $3+$ & 135 & iv & $0+33$ & \\
\hline 24 & 1.43 & & & $4 \cdot 46$ & $11 \cdot 3$ & $11 \cdot 9$ & $14 \cdot 1$ & $16 \cdot 7$ & $21 \cdot 8$ & 22.95 & $25 \cdot 65$ & 13 & 16 & $3+16$ & $2+16$ & 140 & 8 iv & $1+32$ & \\
\hline 25 & 1.53 & & & $4 \cdot 66$ & $11 \cdot 65$ & $12 \cdot 6$ & $14 \cdot 45$ & $17 \cdot 4$ & $22 \cdot 2$ & $23 \cdot 35$ & $26 \cdot 15$ & 15 & 16 & $3+16$ & & 166 & & $1+32$ & \\
\hline 26 & $1 \cdot 35$ & & & $4 \cdot 2$ & $10 \cdot 25$ & $10 \cdot 6$ & $12 \cdot 5$ & $15 \cdot 05$ & $19 \cdot 3$ & $20 \cdot 4$ & $22 \cdot 7$ & 1 & 17 & $3+17$ & & 95 & ii -iii & $1+31$ & \\
\hline 27 & $1 \cdot 6$ & & & $5 \cdot 2$ & $12 \cdot 4$ & $13 \cdot 05$ & 15 & $18 \cdot 5$ & $23 \cdot 9$ & $25 \cdot 2$ & $27 \cdot 9$ & 15 & 16 & $2+16$ & $2+17$ & 165 & $\hat{\sigma} \mathrm{v}$ & $1+33$ & \\
\hline 28 & $1 \cdot 4$ & & & $4 \cdot 25$ & $10 \cdot 35$ & $10 \cdot 85$ & 12.55 & $15 \cdot 3$ & $20 \cdot 05$ & $21 \cdot 1$ & $23 \cdot 6$ & 15 & 17 & $2+16$ & $2+14$ & 104 & iii & $0+32$ & \\
\hline
\end{tabular}

19




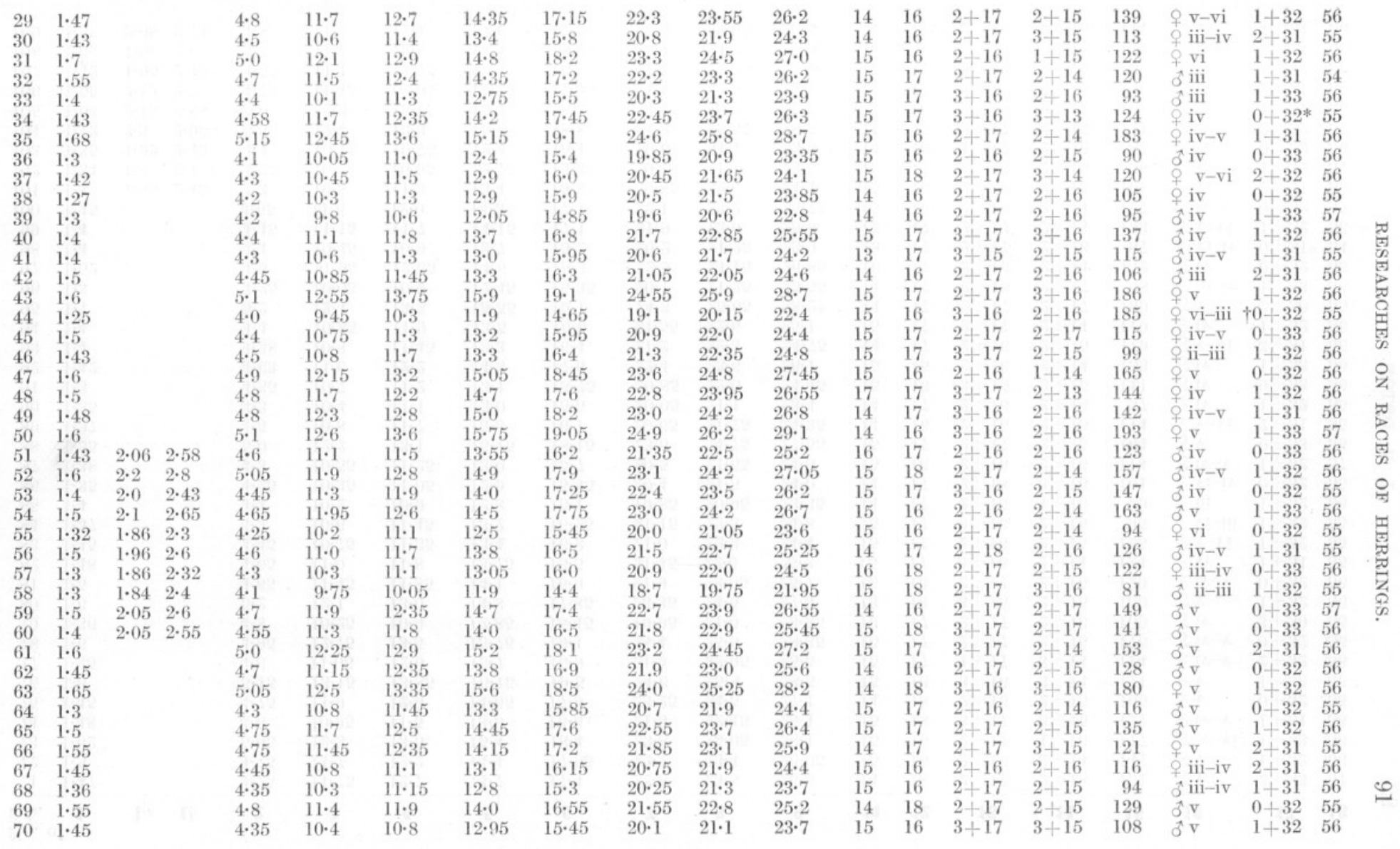




\begin{tabular}{|c|c|c|c|c|c|c|c|c|c|c|c|c|c|c|c|c|c|c|c|}
\hline $\begin{array}{l}\text { No. } \\
\text { fish. }\end{array}$ & 1 & $1 a$ & $1 b$ & 2 & 3 & 4 & 5 & 6 & 7 & 8 & 9 & 11 & 12 & 13 & 14 & 15 & 16 & 17 & 18 \\
\hline 71 & $1 \cdot 47$ & & & $4 \cdot 75$ & $11 \cdot 75$ & $12 \cdot 7$ & $14 \cdot 7$ & $17 \cdot 4$ & $22 \cdot 8$ & $23 \cdot 8$ & $26 \cdot 6$ & 14 & 18 & $2+17$ & $2+18$ & 151 & $\widehat{o v}$ & $2+33$ & 57 \\
\hline 72 & 1.5 & & & $4 \cdot 75$ & $11 \cdot 7$ & $12 \cdot 2$ & $14 \cdot 4$ & $17 \cdot 55$ & $22 \cdot 2$ & $23 \cdot 4$ & $26 \cdot 05$ & 15 & 16 & $2+16$ & $2+14$ & 147 & & $1+32$ & 56 \\
\hline 73 & $1 \cdot 62$ & & & $5 \cdot 05$ & $12 \cdot 3$ & $13 \cdot 5$ & $15 \cdot 4$ & $18 \cdot 4$ & $23 \cdot 8$ & $25 \cdot 05$ & $28 \cdot 1$ & 15 & 16 & $2+17$ & $1+16$ & 177 & $\mathrm{v}-\mathrm{vi}$ & $1+33$ & 56 \\
\hline 75 & 1.45 & & & $4 \cdot 75$ & $11 \cdot 9$ & $12 \cdot 9$ & $14 \cdot 65$ & $17 \cdot 9$ & $23 \cdot 25$ & $24 \cdot 5$ & $27 \cdot 1$ & 14 & 18 & $2+16$ & $2+15$ & 165 & $\mathrm{v}$ & $0+32$ & 55 \\
\hline 76 & $1 \cdot 6$ & & & $5 \cdot 15$ & $13 \cdot 15$ & 13.85 & $16 \cdot 15$ & $19 \cdot 5$ & $24 \cdot 6$ & $25 \cdot 8$ & $28 \cdot 5$ & 15 & 16 & $3+16$ & $3+14$ & 171 & $q \mathrm{v}$ & $1+33$ & 56 \\
\hline 77 & $1 \cdot 45$ & & & $4 \cdot 6$ & $11 \cdot 05$ & $12 \cdot 0$ & $13 \cdot 7$ & $16 \cdot 9$ & $21 \cdot 9$ & $22 \cdot 95$ & $25 \cdot 5$ & 15 & 17 & $1+17$ & $3+14$ & 124 & iv $\mathrm{iv}-\mathrm{v}$ & $2+32$ & 56 \\
\hline 78 & $1 \cdot 6$ & & & $4 \cdot 95$ & $12 \cdot 75$ & 13.5 & $15 \cdot 75$ & $19 \cdot 1$ & $24 \cdot 4$ & $25 \cdot 6$ & $28 \cdot 35$ & 16 & 17 & $2+17$ & $2+16$ & 176 & ôv-v & $0+32$ & 56 \\
\hline 79 & $1 \cdot 36$ & & & $4 \cdot 3$ & $10 \cdot 25$ & $10 \cdot 9$ & $12 \cdot 85$ & $15 \cdot 45$ & $19 \cdot 95$ & $21 \cdot 0$ & $23 \cdot 35$ & 16 & 17 & $2+17$ & & 109 & ô iv & $3+30$ & 55 \\
\hline 80 & $1 \cdot 6$ & & & $5 \cdot 2$ & $12 \cdot 1$ & $13 \cdot 0$ & $14 \cdot 8$ & $17 \cdot 85$ & $23 \cdot 35$ & $24 \cdot 5$ & $27 \cdot 3$ & 13 & 16 & $3+16$ & $2+15$ & 150 & & $1+32$ & 56 \\
\hline 81 & $1 \cdot 5$ & & & $4 \cdot 65$ & $11 \cdot 45$ & $11 \cdot 85$ & $14 \cdot 1$ & $16 \cdot 9$ & $21 \cdot 9$ & $23 \cdot 05$ & $25 \cdot 7$ & 15 & 16 & $2+17$ & $2+16$ & 129 & 우 iv-v & $0+32$ & 56 \\
\hline 82 & $1 \cdot 48$ & & & $4 \cdot 65$ & $11 \cdot 0$ & $11 \cdot 8$ & $13 \cdot 75$ & $16 \cdot 5$ & $21 \cdot 45$ & $22 \cdot 6$ & $25 \cdot 2$ & 15 & 17 & $3+$ & & 124 & i & $1+31$ & 55 \\
\hline 83 & 1.45 & & & $4 \cdot 45$ & $10 \cdot 75$ & $11 \cdot 35$ & $13 \cdot 4$ & $16 \cdot 2$ & $21 \cdot 0$ & $22 \cdot 2$ & $24 \cdot 4$ & 1. & 17 & $2+16$ & $2+15$ & 125 & $v-v i$ & $1+32$ & 55 \\
\hline 84 & $1 \cdot 37$ & & & $4 \cdot 3$ & $10 \cdot 6$ & $11 \cdot 45$ & $13 \cdot 2$ & $16 \cdot 25$ & $21 \cdot 15$ & $22 \cdot 3$ & $24 \cdot 8$ & 15 & 17 & $3+16$ & $2+16$ & 95 & vi-iii & $1+32$ & 55 \\
\hline 85 & $1 \cdot 4$ & & & $4 \cdot 4$ & $10 \cdot 2$ & $10 \cdot 9$ & $12 \cdot 8$ & $15 \cdot 1$ & $19 \cdot 85$ & $20 \cdot 95$ & $23 \cdot 35$ & 14 & 17 & $2+15$ & $1+$ & 99 & iii & $1+32$ & 56 \\
\hline 86 & $1 \cdot 35$ & & & $4 \cdot 25$ & $10 \cdot 35$ & $11 \cdot 05$ & $12 \cdot 9$ & $15 \cdot 45$ & $20 \cdot 4$ & $21 \cdot 5$ & $24 \cdot 0$ & 14 & 17 & $2+18$ & $3+16$ & 101 & vi-iv & $0+32$ & 55 \\
\hline 87 & $1 \cdot 38$ & & & $4 \cdot 3$ & $10 \cdot 55$ & $11 \cdot 25$ & $13 \cdot 0$ & $15 \cdot 7$ & $20 \cdot 5$ & $21 \cdot 65$ & $24 \cdot 0$ & 15 & 16 & $2+16$ & $2+16$ & 114 & ô iv-v & $1+32$ & 57 \\
\hline 88 & $1 \cdot 55$ & & & $5 \cdot 0$ & $12 \cdot 2$ & $13 \cdot 1$ & $15 \cdot 15$ & $18 \cdot 15$ & $23 \cdot 5$ & $24 \cdot 75$ & $27 \cdot 55$ & 15 & 17 & $2+17$ & $2+15$ & 164 & & $0+33$ & 56 \\
\hline 89 & $1 \cdot 37$ & & & $4 \cdot 5$ & $10 \cdot 8$ & $11 \cdot 7$ & 13.5 & $16 \cdot 1$ & $20 \cdot 65$ & $21 \cdot 75$ & $24 \cdot 35$ & 14 & 16 & $2+16$ & $2+$ & 114 & $v-v i$ & $2+31$ & 55 \\
\hline 90 & $1 \cdot 35$ & & & $4 \cdot 3$ & $10 \cdot 4$ & $11 \cdot 1$ & $13 \cdot 0$ & $15 \cdot 5$ & $20 \cdot 05$ & $21 \cdot 1$ & $23 \cdot 4$ & 14 & 17 & $2+16$ & $2+$ & 97 & 우 iv & $1+32$ & 55 \\
\hline 92 & $1 \cdot 42$ & & & $4 \cdot 63$ & $11 \cdot 6$ & $12 \cdot 2$ & $14 \cdot 3$ & $17 \cdot 6$ & $22 \cdot 4$ & $23 \cdot 6$ & $26 \cdot 1$ & 15 & 15 & $2+$ & $2+$ & 153 & ô iv & $1+$ & 56 \\
\hline 93 & $1 \cdot 4$ & & & $4 \cdot 38$ & $10 \cdot 8$ & $11 \cdot 45$ & $13 \cdot 3$ & $16 \cdot 4$ & $21 \cdot 2$ & 22 & $24 \cdot 75$ & 14 & 17 & $2+16$ & $2+15$ & 119 & ô v-vi & $3+31$ & 56 \\
\hline 94 & $1 \cdot 4$ & & & $4 \cdot 4$ & $10 \cdot 85$ & $11 \cdot 9$ & $13 \cdot 5$ & $16 \cdot 6$ & $21 \cdot 55$ & $22 \cdot 6$ & $25 \cdot 1$ & 15 & 17 & $2+17$ & $2+14$ & 119 & iv & $\dagger 1+32$ & 56 \\
\hline 95 & 1.7 & & & $5 \cdot 2$ & $12 \cdot 4$ & $13 \cdot 8$ & $15 \cdot 55$ & $19 \cdot 1$ & $24 \cdot 3$ & $25 \cdot 65$ & $28 \cdot 2^{*}$ & 14 & 17 & $2+17$ & $2+17$ & 199 & $\sqrt{y}$ & $2+30$ & 55 \\
\hline 96 & $1 \cdot 3$ & & & $4 \cdot 25$ & $10 \cdot 05$ & $10 \cdot 9$ & $12 \cdot 45$ & $15 \cdot 35$ & $20 \cdot 1$ & $21 \cdot 25$ & 23 . & 14 & 17 & $2+$ & $2+17$ & 98 & iii-iv & & 56 \\
\hline 97 & 1.52 & & & $4 \cdot 9$ & $11 \cdot 95$ & $12 \cdot 65$ & $14 \cdot 6$ & $18 \cdot 2$ & $22 \cdot 9$ & $24 \cdot 15$ & $26 \cdot 85$ & 16 & 17 & $2+16$ & $2+14$ & 142 & iv & $0+32$ & 55 \\
\hline 98 & $1 \cdot 4$ & & & $4 \cdot 4$ & $10 \cdot 45$ & $10 \cdot 9$ & $12 \cdot 7$ & $15 \cdot 2$ & $20 \cdot 2$ & $21 \cdot 25$ & $23 \cdot 9$ & 13 & 17 & $2+17$ & $2+16$ & 97 & vi-iv & $0+31^{*}$ & 54 \\
\hline 99 & 1.4 & & & $4 \cdot 45$ & $11 \cdot 15$ & $11 \cdot 7$ & $14 \cdot 15$ & $17 \cdot 1$ & $21 \cdot 9$ & $23 \cdot 0$ & $25 \cdot 6$ & 15 & 17 & $2+17$ & $2+14$ & 133 & i v-vi & $1+32$ & 56 \\
\hline 100 & 1.45 & & & $4 \cdot 65$ & $11 \cdot 6$ & $12 \cdot 0$ & $14 \cdot 3$ & $17 \cdot 4$ & $22 \cdot 1$ & $23 \cdot 2$ & $25 \cdot 75$ & 15 & 16 & $2+16$ & $2+14$ & 127 & of iv-v & $0+32$ & 55 \\
\hline 101 & $1 \cdot 4$ & $1 \cdot 85$ & $2 \cdot 45$ & $4 \cdot 3$ & $10 \cdot 8$ & $11 \cdot 3$ & $13 \cdot 4$ & $15 \cdot 8$ & $20 \cdot 85$ & $22 \cdot 0$ & $24 \cdot 4$ & 15 & 16 & $2+17$ & $2+15$ & 101 & 우 vi & $2+31$ & 56 \\
\hline 102 & $1 \cdot 34$ & 1.8 & $2 \cdot 4$ & $4 \cdot 35$ & $10 \cdot 2$ & $10 \cdot 95$ & $12 \cdot 75$ & $15 \cdot 3$ & $20 \cdot 1$ & $21 \cdot 1$ & 23.5 & 15 & 17 & $1+17$ & $2+15$ & 98 & & $1+32$ & 57 \\
\hline 103 & $1 \cdot 35$ & 1.92 & $2 \cdot 43$ & $4 \cdot 4$ & $10 \cdot 15$ & $10 \cdot 75$ & $12 \cdot 4$ & $15 \cdot 1$ & 20 & $21 \cdot 1$ & 23 . & 14 & 16 & $1+17$ & $2+16$ & 85 & & $0+33$ & 56 \\
\hline 104 & $1 \cdot 73$ & $2 \cdot 3$ & $2 \cdot 96$ & $5 \cdot 3$ & $12 \cdot 4$ & $13 \cdot 3$ & $15 \cdot 6$ & $18 \cdot 8$ & $24 \cdot 2$ & 25.55 & $28 \cdot 5$ & 15 & 17 & $2+17$ & $2+15$ & 171 & & $2+32$ & 57 \\
\hline 105 & $1 \cdot 64$ & $2 \cdot 15$ & $2 \cdot 88$ & $5 \cdot 0$ & $12 \cdot 3$ & $13 \cdot 25$ & $15 \cdot 4$ & $18 \cdot 3$ & $23 \cdot 65$ & $25 \cdot 0$ & $27 \cdot 7$ & 14 & 16 & $2+17$ & $2+15$ & 162 & & $1+31$ & 55 \\
\hline 106 & 1.55 & $2 \cdot 13$ & $2 \cdot 7$ & $4 \cdot 75$ & $11 \cdot 45$ & $12 \cdot 05$ & $14 \cdot 25$ & $17 \cdot 05$ & $21 \cdot 8$ & $22 \cdot 9$ & $25 \cdot 7$ & 14 & 17 & $2+17$ & $2+16$ & 163 & iv $-\mathrm{v}$ & $2+31$ & 55 \\
\hline 107 & 1.42 & 1.95 & $2 \cdot 43$ & 4.55 & $11 \cdot 1$ & $11 \cdot 75$ & $13 \cdot 7$ & $16 \cdot 6$ & $21 \cdot 7$ & $22 \cdot 75$ & & 1. & 17 & $2+17$ & & 156 & & & 56 \\
\hline 108 & $1 \cdot 52$ & $1 \cdot 96$ & $2 \cdot 6$ & $4 \cdot 7$ & $11 \cdot 7$ & $12 \cdot 8$ & $14 \cdot 4$ & $18 \cdot 05$ & $23 \cdot 1$ & $24 \cdot 3$ & $27 \cdot 0$ & 1 & 16 & $3+16$ & $1+15$ & 144 & iv-v & $1+31$ & 55 \\
\hline 109 & 1.56 & $2 \cdot 08$ & $2 \cdot 74$ & $4 \cdot 8$ & $11 \cdot 7$ & 12.4 & $14 \cdot 6$ & $17 \cdot 3$ & $22 \cdot 5$ & $23 \cdot 65$ & $26 \cdot 5$ & 14 & 17 & $2+16$ & $3+15$ & 147 & . & $1+31$ & 55 \\
\hline
\end{tabular}




\begin{tabular}{|c|c|c|c|c|c|c|c|c|c|c|c|c|c|c|c|c|c|c|c|}
\hline 110 & 1.7 & $2 \cdot 2$ & $3 \cdot 0$ & $5 \cdot 05$ & $12 \cdot 2$ & $13 \cdot 2$ & $15 \cdot 1$ & $18 \cdot 2$ & $23 \cdot 4$ & $24 \cdot 7$ & $27 \cdot 5$ & 15 & 16 & $2+16$ & $3+14$ & 156 & $\hat{o} \mathrm{v}$ & $2+30$ & 55 \\
\hline 111 & $1 \cdot 54$ & & & $4 \cdot 5$ & $11 \cdot 2$ & $11 \cdot 8$ & $14 \cdot 2$ & $16 \cdot 6$ & $21 \cdot 6$ & $22 \cdot 75$ & $25 \cdot 35$ & 14 & 16 & $3+17$ & $3+14$ & 131 & o iii-iv & $1+31$ & 55 \\
\hline 112 & $1 \cdot 4$ & & & $4 \cdot 25$ & $10 \cdot 45$ & $11 \cdot 7$ & $12 \cdot 9$ & $16 \cdot 2$ & $21 \cdot 0$ & $22 \cdot 2$ & $24 \cdot 65$ & 14 & 17 & $2+16$ & $1+16$ & 114 & q vi & $2+32$ & 56 \\
\hline 113 & $1 \cdot 5$ & & & $4 \cdot 6$ & $11 \cdot 05$ & $12 \cdot 1$ & $13 \cdot 7$ & $16 \cdot 5$ & $21 \cdot 5$ & $22 \cdot 65$ & $25 \cdot 05$ & 14 & 15 & $2+17$ & $2+15$ & 120 & iv $-\mathrm{v}$ & $1+32$ & 55 \\
\hline 114 & 1.58 & & & $4 \cdot 74$ & $11 \cdot 2$ & $12 \cdot 15$ & $14 \cdot 25$ & $17 \cdot 05$ & $22 \cdot 4$ & $23 \cdot 55$ & $26 \cdot 3$ & 15 & 17 & $2+18$ & $1+17$ & 143 & ôii-iv & $1+32$ & 56 \\
\hline 115 & $1 \cdot 4$ & & & $4 \cdot 65$ & $11 \cdot 1$ & $11 \cdot 8$ & $13 \cdot 9$ & $16 \cdot 6$ & $21 \cdot 5$ & $22 \cdot 6$ & $25 \cdot 3$ & 15 & 18 & $3+16$ & $2+14$ & 116 & ô iv-v & $0+32$ & 55 \\
\hline 116 & $1 \cdot 6$ & & & $4 \cdot 9$ & $11 \cdot 7$ & $12 \cdot 8$ & $14 \cdot 8$ & $17 \cdot 7$ & $22 \cdot 9$ & $24 \cdot 05$ & $26 \cdot 9$ & 15 & 16 & $2+17$ & $2+15$ & 159 & $\mathrm{o}^{1} \mathrm{~V}$ & $1+32$ & 56 \\
\hline 117 & 1.58 & & & $5 \cdot 1$ & $12 \cdot 75$ & $14 \cdot 0$ & $15 \cdot 9$ & $19 \cdot 15$ & $24 \cdot 65$ & $25 \cdot 95$ & $28 \cdot 85$ & 14 & 18 & $2+17$ & $2+16$ & 213 & $\sigma^{4} \mathrm{~V}$ & $2+32$ & 56 \\
\hline 118 & $1 \cdot 45$ & & & $4 \cdot 65$ & $11 \cdot 4$ & $12 \cdot 25$ & $14 \cdot 5$ & $17 \cdot 15$ & $22 \cdot 2$ & $23 \cdot 2$ & $26 \cdot 05$ & 14 & 17 & $2+18$ & $2+15$ & 122 & ô $\mathrm{v}$ & $\dagger 0+33$ & 56 \\
\hline 119 & 1.5 & & & $4 \cdot 85$ & $12 \cdot 2$ & $13 \cdot 0$ & $15 \cdot 15$ & $18 \cdot 5$ & $23 \cdot 8$ & $25 \cdot 0$ & $27 \cdot 8$ & 15 & 17 & $2+17$ & $2+16$ & 172 & ov & $0+33$ & 56 \\
\hline 120 & $1 \cdot 63$ & & & $5 \cdot 1$ & $12 \cdot 8$ & $13 \cdot 4$ & $15 \cdot 7$ & $19 \cdot 05$ & $24 \cdot 25$ & $25 \cdot 45$ & $28 \cdot 3$ & 14 & 17 & $2+16$ & $2+15$ & 176 & iv-r & $0+32$ & 55 \\
\hline 121 & $1 \cdot 4$ & & & $4 \cdot 65$ & $11 \cdot 05$ & $11 \cdot 55$ & $13 \cdot 7$ & $16 \cdot 35$ & $21 \cdot 3$ & $22 \cdot 45$ & $24 \cdot 95$ & 14 & 18 & $2+17$ & $3+14$ & 120 & ô iv & $1+33$ & 56 \\
\hline 122 & $1 \cdot 33$ & & & $4 \cdot 5$ & $11 \cdot 0$ & $11 \cdot 8$ & $13 \cdot 6$ & $16 \cdot 8$ & $21 \cdot 65$ & $22 \cdot 75$ & $25 \cdot 5$ & 16 & 16 & $2+16$ & $1+13$ & 117 & i iv-v & $0+33$ & 55 \\
\hline 123 & $1 \cdot 46$ & & & $4 \cdot 76$ & $11 \cdot 3$ & $11 \cdot 7$ & $13 \cdot 85$ & $16 \cdot 55$ & $21 \cdot 8$ & $22 \cdot 85$ & $25 \cdot 5$ & 14 & 17 & $2+17$ & $2+14$ & 128 & $\mathrm{v}$ & $1+32$ & 55 \\
\hline 124 & 1.5 & & & $4 \cdot 5$ & $10 \cdot 9$ & $11 \cdot 5$ & $13 \cdot 55$ & $16 \cdot 3$ & $21 \cdot 2$ & $22 \cdot 3$ & $25 \cdot 0$ & 15 & 16 & $2+16$ & $2+16$ & 123 & iv-vi & $1+31$ & 55 \\
\hline 125 & 1.5 & & & 4.55 & $11 \cdot 4$ & $12 \cdot 15$ & $14 \cdot 1$ & $17 \cdot 1$ & $22 \cdot 1$ & $23 \cdot 25$ & $25 \cdot 9$ & 15 & 17 & $3+17$ & $3+16$ & 121 & iv & $1+33^{*}$ & 57 \\
\hline 126 & $1 \cdot 7$ & & & $5 \cdot 3$ & $12 \cdot 9$ & $13 \cdot 5$ & $15 \cdot 85$ & $19 \cdot 2$ & $24 \cdot 7$ & $25 \cdot 8$ & $28 \cdot 9$ & 15 & 16 & $2+16$ & $2+16$ & 174 & $\delta \mathrm{v}-\mathrm{vi}$ & $2+32$ & 56 \\
\hline 127 & 1.5 & & & $4 \cdot 8$ & $12 \cdot 0$ & $13 \cdot 0$ & $14 \cdot 9$ & $18 \cdot 7$ & $23 \cdot 55$ & $24 \cdot 7$ & $27 \cdot 4$ & 15 & 17 & $3+17$ & $2+15$ & 169 & ôv & $0+33$ & 56 \\
\hline 128 & $1 \cdot 4$ & & & $4 \cdot 47$ & $11 \cdot 5$ & $12 \cdot 4$ & $14 \cdot 2$ & $17 \cdot 4$ & 22.5 & $23 \cdot 6$ & $26 \cdot 3$ & 15 & 16 & $2+17$ & $2+14$ & 144 & & $2+31$ & 56 \\
\hline 129 & 1.5 & & & $4 \cdot 5$ & $10 \cdot 8$ & $11 \cdot 6$ & $13 \cdot 5$ & $16 \cdot 3$ & $21 \cdot 45$ & $22 \cdot 5$ & $24 \cdot 95$ & 15 & 17 & $2+17$ & $2+15$ & 120 & vi & $0+32$ & 56 \\
\hline 130 & $1 \cdot 6$ & & & $5 \cdot 1$ & $12 \cdot 4$ & $12 \cdot 9$ & $15 \cdot 5$ & $18 \cdot 45$ & $23 \cdot 8$ & $25 \cdot 05$ & $28 \cdot 05$ & 15 & 17 & $2+18$ & $2+16$ & 189 & $\hat{\delta} \mathrm{v}-\mathrm{vi}$ & $1+32$ & 56 \\
\hline 131 & $1 \cdot 4$ & & & $4 \cdot 5$ & $10 \cdot 9$ & $11 \cdot 3$ & $13 \cdot 6$ & $16 \cdot 1$ & $21 \cdot 15$ & $22 \cdot 25$ & $24 \cdot 8$ & 15 & 17 & $2+17$ & $2+16$ & 114 & of iv-v & $0+34$ & 56 \\
\hline 132 & $1 \cdot 55$ & & & $4 \cdot 75$ & $11 \cdot 4$ & $11 \cdot 9$ & $14 \cdot 05$ & $16 \cdot 8$ & $21 \cdot 7$ & $22 \cdot 85$ & $25 \cdot 6$ & 15 & 17 & $2+17$ & $2+15$ & 131 & $\mathrm{iv}-\mathrm{v}$ & $2+31$ & 55 \\
\hline 133 & $1 \cdot 38$ & & & $4 \cdot 35$ & $10 \cdot 55$ & $11 \cdot 4$ & $12 \cdot 95$ & $15 \cdot 75$ & $20 \cdot 65$ & $21 \cdot 7$ & $24 \cdot 2$ & 14 & 17 & $2+16$ & $2+16$ & 112 & $\mathrm{iv}-\mathrm{v}$ & $+0+33$ & 56 \\
\hline 134 & $1 \cdot 65$ & & & $4 \cdot 8$ & $11 \cdot 2$ & $12 \cdot 3$ & $14 \cdot 25$ & $17 \cdot 2$ & $22 \cdot 7$ & $23 \cdot 9$ & $26 \cdot 7$ & 14 & 16 & $2+17$ & $2+16$ & 168 & $\mathrm{v}$ & $0+32$ & 55 \\
\hline 135 & $1 \cdot 4$ & & & $4 \cdot 5$ & $10 \cdot 65$ & $11 \cdot 4$ & $13 \cdot 2$ & $16 \cdot 2$ & $21 \cdot 1$ & $22 \cdot 1$ & $24 \cdot 7$ & 15 & 18 & $3+16$ & $2+15$ & 124 & iv & $1+33$ & 57 \\
\hline 136 & $1 \cdot 6$ & & & $5 \cdot 15$ & $12 \cdot 0$ & $12 \cdot 8$ & $14 \cdot 9$ & $17 \cdot 8$ & $23 \cdot 0$ & $24 \cdot 25$ & $27 \cdot 05$ & 14 & 16 & $2+16$ & $2+15$ & 132 & ôii & $1+31$ & 56 \\
\hline 137 & 1.5 & & & $4 \cdot 7$ & $11 \cdot 4$ & $12 \cdot 25$ & $14 \cdot 1$ & $17 \cdot 15$ & $22 \cdot 05$ & $23 \cdot 25$ & $25 \cdot 95$ & 14 & 16 & $2+16$ & $1+15$ & 137 & o & $2+32$ & 57 \\
\hline 138 & $1 \cdot 7$ & & & $5 \cdot 0$ & $12 \cdot 4$ & $13 \cdot 3$ & $15 \cdot 3$ & $18 \cdot 4$ & $23 \cdot 75$ & $25 \cdot 1$ & $27 \cdot 9$ & 15 & 17 & $3+17$ & $2+16$ & 172 & ô $\mathrm{v}$ & $1+32$ & 56 \\
\hline 139 & 1.5 & & & $4 \cdot 7$ & $11 \cdot 4$ & $11 \cdot 8$ & $14 \cdot 2$ & $16 \cdot 7$ & $21 \cdot 85$ & $23 \cdot 0$ & $25 \cdot 65$ & 15 & 17 & $2+17$ & $2+15$ & 134 & o iii-iv & $2+30 *$ & 56 \\
\hline 140 & $1 \cdot 7$ & & & $5 \cdot 1$ & $12 \cdot 0$ & $13 \cdot 5$ & $14 \cdot 9$ & $18 \cdot 5$ & $23 \cdot 5$ & $24 \cdot 7$ & $27 \cdot 55$ & 15 & 17 & $3+16$ & $2+15$ & 170 & $\$ \mathrm{v}$ & $1+32$ & 56 \\
\hline 141 & $1 \cdot 5$ & & & $4 \cdot 5$ & $10 \cdot 7$ & $11 \cdot 6$ & $13 \cdot 3$ & $15 \cdot 8$ & $20 \cdot 9$ & $21 \cdot 95$ & $24 \cdot 55$ & 15 & 16 & $2+16$ & $3+15$ & 115 & iv $\mathrm{iv}-\mathrm{v}$ & $1+31$ & 56 \\
\hline 142 & $1 \cdot 4$ & & & $4 \cdot 5$ & $10 \cdot 85$ & $11 \cdot 6$ & $13 \cdot 7$ & $16 \cdot 5$ & $21 \cdot 5$ & $22 \cdot 5$ & $24 \cdot 9$ & 14 & 17 & $2+17$ & $2+17$ & 119 & ô iv & $0+32$ & 56 \\
\hline 143 & $1 \cdot 5$ & & & $4 \cdot 86$ & $11 \cdot 6$ & $12 \cdot 4$ & $14 \cdot 35$ & $17 \cdot 5$ & $22 \cdot 75$ & $23 \cdot 95$ & $26 \cdot 65$ & 14 & 17 & $2+17$ & $2+16$ & 157 & vi & $+0+34$ & 56 \\
\hline 144 & $1 \cdot 55$ & & & $4 \cdot 65$ & $11 \cdot 7$ & $12 \cdot 1$ & $14 \cdot 55$ & $17 \cdot 8$ & $23 \cdot 0$ & $24 \cdot 15$ & $26 \cdot 9$ & 15 & 17 & $2+16$ & $3+15$ & 144 & 우 vi-iv & $0+32$ & 56 \\
\hline 145 & 1.5 & & & $4 \cdot 7$ & $11 \cdot 55$ & $12 \cdot 45$ & $14 \cdot 3$ & $17 \cdot 5$ & $22 \cdot 8$ & $24 \cdot 0$ & $26 \cdot 75$ & 14 & 16 & $2+16$ & $3+15$ & 146 & $\hat{o v}$ & $2+30$ & 55 \\
\hline 146 & $1 \cdot 47$ & & & $4 \cdot 6$ & $11 \cdot 0$ & $11 \cdot 7$ & $13 \cdot 65$ & $16 \cdot 6$ & $21 \cdot 95$ & $22 \cdot 95$ & $25 \cdot 65$ & 15 & 16 & $2+17$ & $2+16$ & 123 & o iv & $0+33$ & 56 \\
\hline 147 & $1 \cdot 44$ & & & $4 \cdot 73$ & $11 \cdot 5$ & $11 \cdot 75$ & $14 \cdot 3$ & $16 \cdot 9$ & $22 \cdot 05$ & $23 \cdot 1$ & $25 \cdot 8$ & 15 & 16 & $2+17$ & $2+15$ & 132 & iv & $+0+32$ & 56 \\
\hline 148 & 1.5 & & & $4 \cdot 7$ & $11 \cdot 55$ & $12 \cdot 7$ & $14 \cdot 25$ & $17 \cdot 8$ & $22 \cdot 85$ & $24 \cdot 1$ & $26 \cdot 6$ & 15 & 16 & $2+18$ & $2+15$ & 130 & ôi & $1+32$ & 56 \\
\hline 149 & 1.5 & & & $4 \cdot 6$ & $11 \cdot 5$ & $12 \cdot 2$ & $14 \cdot 3$ & $17 \cdot 45$ & $22 \cdot 75$ & $23 \cdot 9$ & $26 \cdot 55$ & 15 & 16 & $3+16$ & $2+16$ & 159 & 우 & $1+32$ & 56 \\
\hline 150 & $1 \cdot 5$ & & & $4 \cdot 7$ & $12 \cdot 1$ & $13 \cdot 0$ & $14 \cdot 9$ & $18 \cdot 1$ & $23 \cdot 5$ & $24 \cdot 7$ & $27 \cdot 5$ & 15 & 17 & $3+16$ & $2+16$ & 164 & iv-v & $1+32$ & 56 \\
\hline 151 & $1 \cdot 35$ & & & $4 \cdot 3$ & $10 \cdot 3$ & $11 \cdot 3$ & $12 \cdot 7$ & $15 \cdot 5$ & $20 \cdot 45$ & $21 \cdot 55$ & $24 \cdot 0$ & 14 & 16 & $2+16$ & $2+15$ & 103 & iii & $0+34$ & 57 \\
\hline
\end{tabular}




\begin{tabular}{|c|c|c|c|c|c|c|c|c|c|c|c|c|c|c|c|c|c|c|c|}
\hline $\begin{array}{l}\text { No. o } \\
\text { fish. }\end{array}$ & 1 & $1 a$ & $1 b$ & 2 & 3 & 4 & 5 & 6 & 7 & 8 & 9 & 11 & 12 & 13 & 14 & 15 & 16 & 17 & 18 \\
\hline 152 & $1 \cdot 24$ & & & $4 \cdot 15$ & $9 \cdot 95$ & $11 \cdot 1$ & $12 \cdot 4$ & $15 \cdot 3$ & $20 \cdot 0$ & $21 \cdot 0$ & $23 \cdot 5$ & 15 & 17 & $2+17$ & $3+15$ & 103 & ơ iii-iv & $1+32$ & 56 \\
\hline 153 & 1.45 & & & $4 \cdot 45$ & $10 \cdot 6$ & $11 \cdot 8$ & 13.55 & $16 \cdot 5$ & $21 \cdot 5$ & $22 \cdot 6$ & $25 \cdot 3$ & 14 & 17 & $3+17$ & $2+15$ & 127 & iv $-\mathrm{v}$ & $1+32$ & 56 \\
\hline 154 & $1 \cdot 43$ & & & $4 \cdot 6$ & $11 \cdot 55$ & $12 \cdot 3$ & $14 \cdot 45$ & $17 \cdot 6$ & $22 \cdot 8$ & $23 \cdot 9$ & $26 \cdot 7$ & 15 & 16 & $2+16$ & $2+16$ & 152 & 우 $\mathbf{v}$ & $1+32$ & 56 \\
\hline 155 & $1 \cdot 4$ & & & $4 \cdot 85$ & $11 \cdot 8$ & $12 \cdot 5$ & $14 \cdot 65$ & $17 \cdot 65$ & $22 \cdot 95$ & $24 \cdot 2$ & 26.95 & 15 & 17 & $3+16$ & $2+17$ & 171 & iv-v & $1+32$ & 56 \\
\hline 156 & $1 \cdot 65$ & & & $5 \cdot 05$ & $12 \cdot 4$ & $12 \cdot 9$ & $15 \cdot 25$ & $18 \cdot 4$ & $23 \cdot 55$ & $24 \cdot 8$ & $27 \cdot 8$ & 15 & 16 & $2+17$ & $2+15$ & 171 & i+ $\mathbf{v}$ & $1+32$ & 56 \\
\hline 157 & $1 \cdot 47$ & & & $4 \cdot 7$ & $11 \cdot 35$ & $11 \cdot 7$ & $14 \cdot 15$ & $16 \cdot 7$ & $22 \cdot 1$ & $23 \cdot 2$ & $25 \cdot 75$ & 16 & 17 & $2+17$ & $2+14$ & 119 & $\sigma^{-1} \mathrm{iii}$ & $1+0+32$ & 57 \\
\hline 158 & 1.5 & & & $4 \cdot 6$ & $11 \cdot 0$ & $12 \cdot 15$ & $13 \cdot 65$ & $16 \cdot 7$ & $21 \cdot 75$ & $22 \cdot 8$ & $25 \cdot 4$ & 14 & 17 & $2+16$ & $2+15$ & 117 & on iv & $0+32$ & 55 \\
\hline 159 & $1 \cdot 63$ & & & $4 \cdot 9$ & $11 \cdot 6$ & $12 \cdot 45$ & $14 \cdot 65$ & $17 \cdot 6$ & $22 \cdot 8$ & $23 \cdot 95$ & $26 \cdot 85$ & 14 & 15 & $2+17$ & $2+16$ & 137 & o $\mathrm{iii}$ & $1+32$ & 56 \\
\hline 160 & $1 \cdot 5$ & & & $4 \cdot 55$ & $10 \cdot 8$ & $11 \cdot 75$ & $13 \cdot 4$ & $16 \cdot 35$ & $21 \cdot 3$ & $22 \cdot 45$ & $25 \cdot 05$ & 15 & 17 & $2+18$ & $2+16$ & 107 & o vi-iv & $2+31$ & 55 \\
\hline 161 & $1 \cdot 4$ & & & $4 \cdot 4$ & $11 \cdot 05$ & $11 \cdot 7$ & $13 \cdot 5$ & $17 \cdot 0$ & $21 \cdot 4$ & $22 \cdot 6$ & $25 \cdot 3$ & 15 & 16 & $2+16$ & $3+14$ & 138 & $\hat{o} \mathrm{v}$ & $1+31$ & *? \\
\hline 162 & $1 \cdot 44$ & & & $4 \cdot 6$ & $11 \cdot 35$ & $12 \cdot 35$ & $14 \cdot 25$ & $17 \cdot 7$ & 22.55 & $23 \cdot 75$ & $26 \cdot 3$ & 14 & 16 & $2+16$ & $2+16$ & 157 & ㅇiv-v & $1+32$ & 56 \\
\hline 163 & $1 \cdot 43$ & & & $4 \cdot 35$ & $10 \cdot 8$ & $11 \cdot 65$ & $13 \cdot 25$ & $16 \cdot 3$ & $21 \cdot 05$ & $22 \cdot 2$ & $24 \cdot 6$ & 14 & 16 & $2+16$ & $2+14$ & 113 & ôii-iv & $1+32$ & 56 \\
\hline 164 & $1 \cdot 33$ & & & $4 \cdot 5$ & $10 \cdot 8$ & $11 \cdot 45$ & $13 \cdot 9$ & $16 \cdot 5$ & $21 \cdot 45$ & $22 \cdot 55$ & $25 \cdot 25$ & 15 & 16 & $2+17$ & $2+16$ & 134 & ôv-vi & $1+32$ & 55 \\
\hline 165 & $1 \cdot 72$ & & & $5 \cdot 05$ & $12 \cdot 75$ & $13 \cdot 05$ & $15 \cdot 5$ & $18 \cdot 65$ & $24 \cdot 0$ & $25 \cdot 3$ & $28 \cdot 2$ & 15 & 17 & $2+16$ & $3+15$ & 185 & क $\mathrm{v}$ & $1+32$ & 56 \\
\hline 166 & $1 \cdot 35$ & & & $4 \cdot 5$ & $11 \cdot 0$ & $10 \cdot 9$ & $13 \cdot 4$ & $16 \cdot 2$ & $21 \cdot 4$ & $22 \cdot 45$ & $25 \cdot 1$ & 16 & 17. & $2+16$ & $2+16$ & 121 & o vi & $2+33$ & 56 \\
\hline 167 & $1 \cdot 36$ & & & $4 \cdot 14$ & $9 \cdot 8$ & $10 \cdot 75$ & $12 \cdot 3$ & $14 \cdot 8$ & $19 \cdot 35$ & $20 \cdot 4$ & 22.9 : & +-+11 & 17 & $2+16$ & $2+16$ & 88 & $\hat{\mathrm{o}} \mathrm{V}$ & $1+31$ & 55 \\
\hline 168 & $1 \cdot 55$ & & & $4 \cdot 55$ & $11 \cdot 0$ & $11 \cdot 45$ & $13 \cdot 6$ & $16 \cdot 5$ & $21 \cdot 4$ & $22 \cdot 5$ & $25 \cdot 0$ & 15 & 17 & $2+16$ & $2+16$ & 110 & ôv-v & $0+33$ & 56 \\
\hline 169 & $1 \cdot 43$ & & & $4 \cdot 55$ & $11 \cdot 05$ & $11 \cdot 45$ & $13 \cdot 9$ & $16 \cdot 65$ & $21 \cdot 6$ & $22 \cdot 75$ & $25 \cdot 6$ & 15 & 17 & $2+17$ & $2+16$ & 138 & o v & $1+32$ & 55 \\
\hline 170 & $1 \cdot 6$ & & & $4 \cdot 65$ & $11 \cdot 5$ & $12 \cdot 4$ & $14 \cdot 1$ & $17 \cdot 3$ & $22 \cdot 5$ & $23 \cdot 7$ & $26 \cdot 4$ & 15 & 16 & $2+16$ & $2+14$ & 156 & q vi & $1+32$ & 56 \\
\hline 171 & $1 \cdot 4$ & & & $4 \cdot 5$ & $11 \cdot 25$ & $12 \cdot 05$ & $14 \cdot 0$ & $17 \cdot 2$ & $22 \cdot 2$ & $23 \cdot 35$ & $25 \cdot 9$ & 16 & 18 & $2+16$ & $2+14$ & 146 & ô $\mathrm{iv}-\mathrm{v}$ & $1+33$ & 56 \\
\hline 172 & $1 \cdot 4$ & & & $4 \cdot 35$ & $10 \cdot 35$ & $11 \cdot 1$ & $12 \cdot 85$ & $15 \cdot 8$ & $20 \cdot 5$ & $21 \cdot 65$ & $23 \cdot 9$ & 15 & 17 & $2+17$ & $2+15$ & 108 & iv $\mathrm{iv}$ - & $\dagger 0+33$ & 56 \\
\hline 173 & $1 \cdot 4$ & & & $4 \cdot 4$ & $10 \cdot 3$ & $10 \cdot 9$ & $12 \cdot 85$ & $15 \cdot 45$ & $20 \cdot 25$ & $21 \cdot 25$ & $23 \cdot 8$ & 15 & 16 & $2+18$ & $2+14$ & 94 & vi-iv & $1+32$ & 56 \\
\hline 174 & $1 \cdot 45$ & & & $4 \cdot 6$ & $11 \cdot 3$ & $12 \cdot 0$ & $13 \cdot 85$ & $17 \cdot 1$ & $22 \cdot 3$ & $23 \cdot 4$ & $26 \cdot 05$ & 16 & 16 & $2+16$ & $3+13$ & 128 & o vi & $1+33$ & 56 \\
\hline 175 & $1 \cdot 46$ & & & $4 \cdot 6$ & $11 \cdot 4$ & $11 \cdot 9$ & $14 \cdot 1$ & $16 \cdot 75$ & $21 \cdot 65$ & $22 \cdot 75$ & $25 \cdot 4$ & 14 & 17 & $3+16$ & $2+16$ & 154 & ㅇ v & $1+31$ & 55 \\
\hline 176 & 1.5 & & & $4 \cdot 75$ & $11 \cdot 45$ & $11 \cdot 9$ & $14 \cdot 25$ & $17 \cdot 15$ & $22 \cdot 4$ & 23.55 & $26 \cdot 2$ & 15 & 16 & $3+17$ & $2+16$ & 131 & iv $\mathrm{iv}-\mathrm{v}$ & $1+31$ & 55 \\
\hline 177 & $1 \cdot 4$ & & & $4 \cdot 25$ & $10 \cdot 15$ & $11 \cdot 05$ & $12 \cdot 7$ & $15 \cdot 7$ & $20 \cdot 15$ & $21 \cdot 25$ & $23 \cdot 7$ & 16 & 15 & $2+17$ & $2+15$ & 107 & & $0+32$ & 56 \\
\hline 178 & $1 \cdot 5$ & & & $4 \cdot 65$ & $11 \cdot 5$ & $12 \cdot 0$ & $14 \cdot 15$ & $16 \cdot 5$ & $22 \cdot 0$ & $23 \cdot 15$ & $25 \cdot 65$ & 14 & 16 & $2+17$ & $2+17$ & 111 & o vii & $1+32$ & 56 \\
\hline 179 & $1 \cdot 48$ & & & $4 \cdot 7$ & $11 \cdot 1$ & $12 \cdot 15$ & $13 \cdot 85$ & $16 \cdot 6$ & $21 \cdot 75$ & $22 \cdot 95$ & $25 \cdot 7$ & 15 & 17 & $3+16$ & $2+15$ & 129 & o iv-v & $0+31$ & 56 \\
\hline 180 & $1 \cdot 6$ & & & $5 \cdot 1$ & $12 \cdot 3$ & $13 \cdot 1$ & $15 \cdot 4$ & $18 \cdot 7$ & $23 \cdot 9$ & $25 \cdot 2$ & $27 \cdot 9$ & 15 & 18 & $2+17$ & $2+15$ & 154 & $\widehat{\delta} \mathbf{v}-\mathbf{v i}$ & $1+32$ & 56 \\
\hline 181 & 1.5 & & & $4 \cdot 85$ & $11 \cdot 85$ & $12 \cdot 65$ & $14 \cdot 75$ & $17 \cdot 55$ & 22.55 & $23 \cdot 7$ & $26 \cdot 3$ & 15 & 15 & $2+17$ & $2+15$ & 140 & q $\mathbf{v}$ & $1+32$ & 56 \\
\hline 182 & 1.4 & & & $4 \cdot 6$ & $10 \cdot 6$ & $11 \cdot 2$ & $13 \cdot 1$ & $16 \cdot 05$ & $20 \cdot 6$ & $21 \cdot 7$ & $24 \cdot 15$ & 16 & 16 & $2+17$ & $2+15$ & 94 & $\hat{o}^{\wedge} \mathrm{iii}$ & $2+31$ & 56 \\
\hline 183 & $1 \cdot 4$ & & & $4 \cdot 5$ & $11 \cdot 1$ & $11 \cdot 9$ & $13 \cdot 85$ & $16 \cdot 75$ & $21 \cdot 85$ & $22 \cdot 95$ & $25 \cdot 4$ & 15 & 17 & $3+16$ & $2+15$ & 122 & o vi-iii & $0+32$ & 55 \\
\hline 184 & 1.8 & & & $5 \cdot 3$ & 12.85 & $13 \cdot 8$ & $15 \cdot 9$ & $18 \cdot 8$ & $24 \cdot 35$ & $25 \cdot 65$ & $28 \cdot 5$ & 14 & 18 & $3+17$ & $3+16$ & 163 & $i \mathbf{v}$ & $1+32$ & 56 \\
\hline 185 & $1 \cdot 65$ & & & $5 \cdot 1$ & $12 \cdot 05$ & $13 \cdot 3$ & $14 \cdot 95$ & $18 \cdot 1$ & $23 \cdot 25$ & $24 \cdot 4$ & $27 \cdot 3$ & 14 & 17 & $3+16$ & $2+15$ & 158 & $\delta^{\hat{N}} \mathrm{v}-\mathrm{vi}$ & $0+31$ & 55 \\
\hline 186 & $1 \cdot 38$ & & & $4 \cdot 33$ & $9 \cdot 8$ & $10 \cdot 45$ & $12 \cdot 2$ & $14 \cdot 8$ & $19 \cdot 45$ & $20 \cdot 45$ & $22 \cdot 8$ & 15 & 17 & $2+16$ & $2+16$ & 90 & $\hat{\delta} \mathrm{v}-\mathrm{vi}$ & $0+33$ & 56 \\
\hline 187 & $1 \cdot 5$ & & & $4 \cdot 6$ & $11 \cdot 0$ & $12 \cdot 3$ & $13 \cdot 5$ & $16 \cdot 85$ & $21 \cdot 8$ & $22 \cdot 95$ & $25 \cdot 7$ & 15 & 17 & $3+16$ & $3+16$ & 126 & $\widehat{o} \mathrm{iv}-\mathrm{v}$ & $0+32$ & 56 \\
\hline 188 & $1 \cdot 3$ & & & $4 \cdot 0$ & $9 \cdot 85$ & $10 \cdot 95$ & $12 \cdot 15$ & $15 \cdot 2$ & $19 \cdot 55$ & $20 \cdot 55$ & $22 \cdot 95$ & 15 & 18 & $3+16$ & $2+16$ & 97 & oii-iv & $+0+32$ & 55 \\
\hline 189 & $1 \cdot 4$ & & & $4 \cdot 45$ & $10 \cdot 55$ & $11 \cdot 15$ & $13 \cdot 15$ & $15 \cdot 9$ & $20 \cdot 85$ & $21 \cdot 95$ & $24 \cdot 4$ & 15 & 16 & $2+17$ & $2+15$ & 101 & iv & $1+32$ & 56 \\
\hline
\end{tabular}




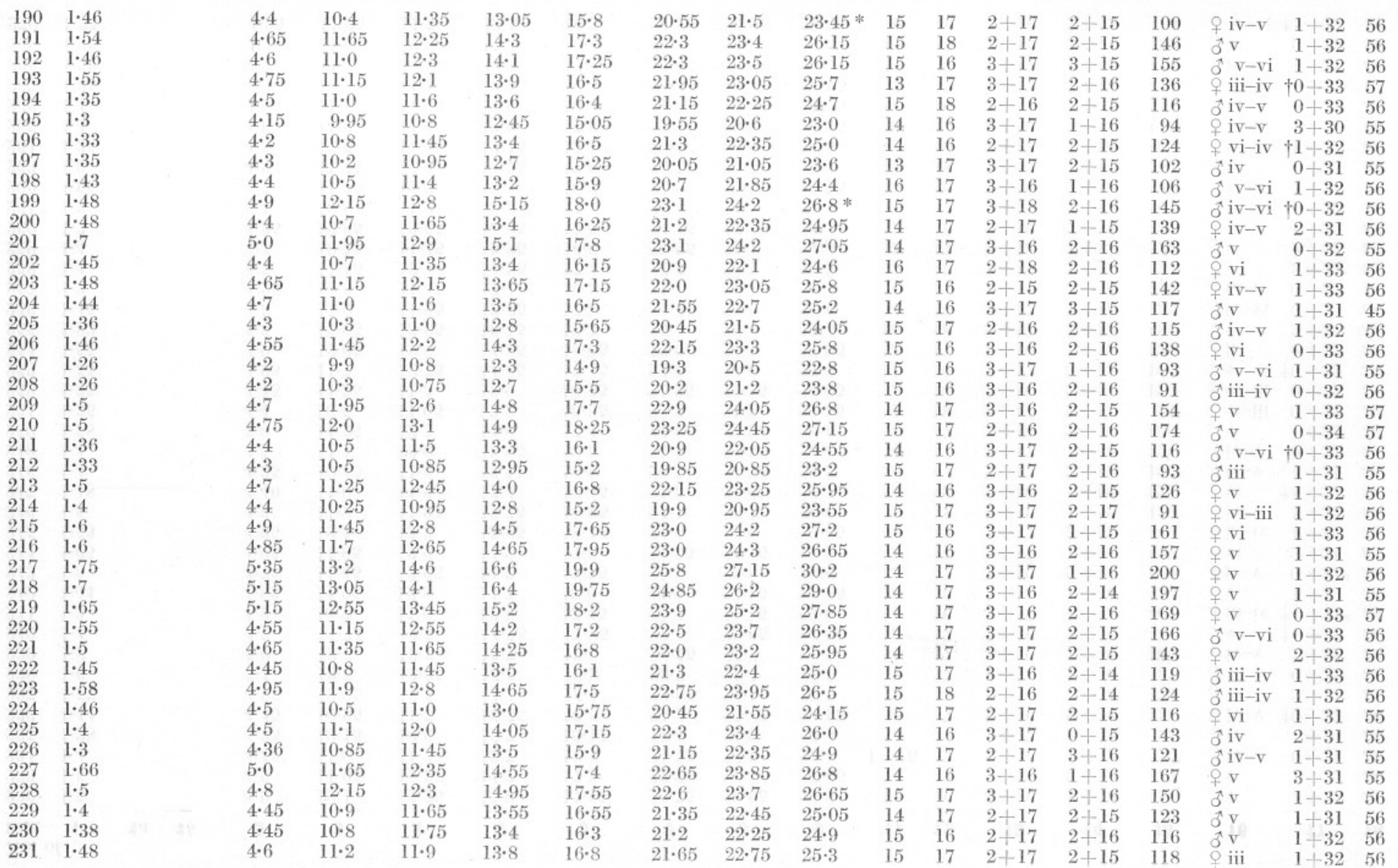




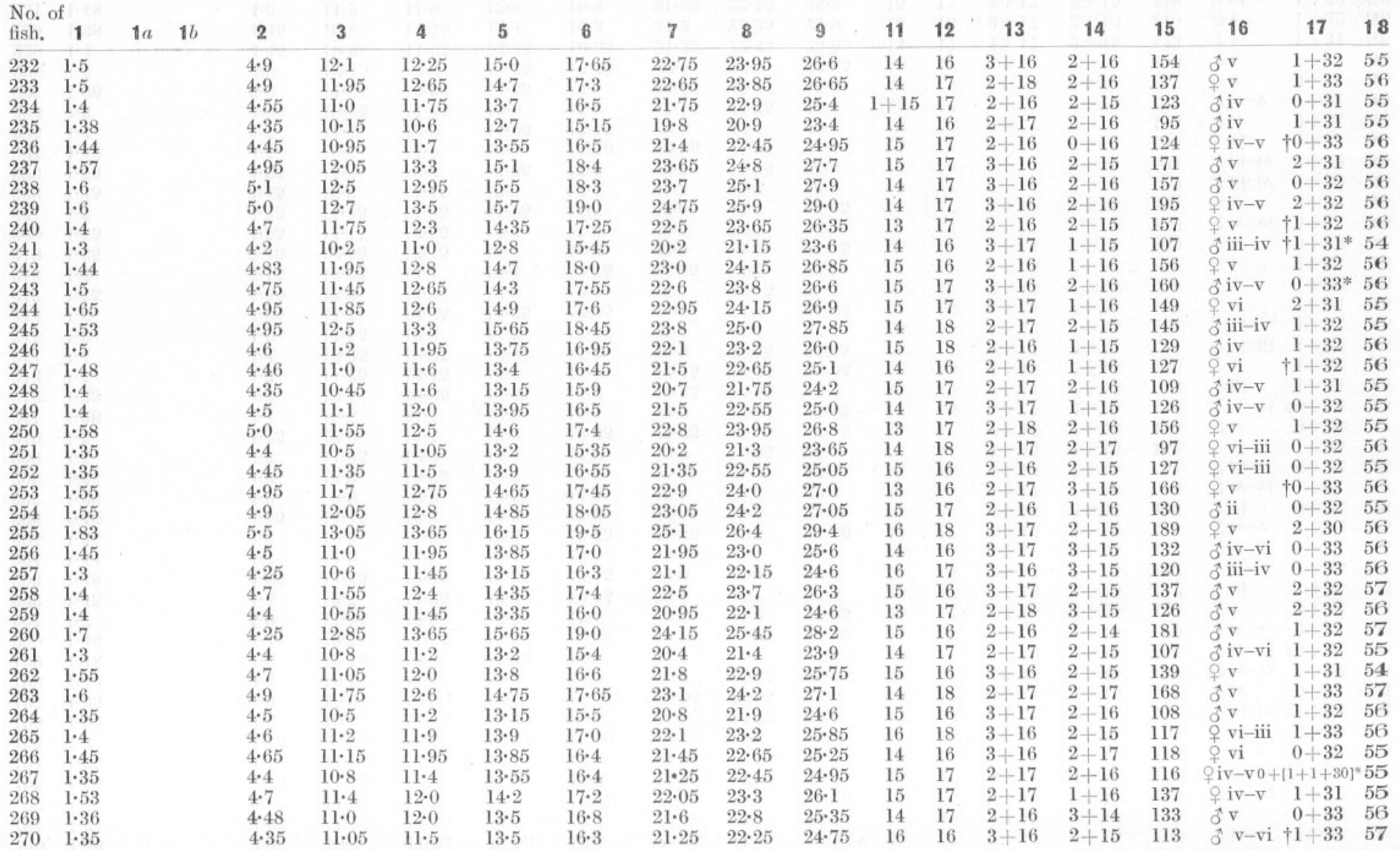


$21 \cdot 1 \quad 22 \cdot 25 \quad 24 \cdot 85$ $23 \cdot 4 \quad 24 \cdot 7$ $20 \cdot 65 \quad 21 \cdot 7$ $21 \cdot 7 \quad 22 \cdot 8$ $19 \cdot 55 \quad 20 \cdot 6$ $21 \cdot 45 \quad 22 \cdot 55$ $21 \cdot 7 \quad 22 \cdot 9$ $24 \cdot 3 \quad 25 \cdot 75$ $21 \cdot 95 \quad 23 \cdot 05$ $23 \cdot 1 \quad 24 \cdot 35$ $21.5 \quad 22.6$ $20.9 \quad 22.1$ $21 \cdot 25 \quad 22 \cdot 35$ $\begin{array}{ll}19 \cdot 7 & 20 \cdot 75 \\ 20 \cdot 1 & 21 \cdot 15\end{array}$ $20 \cdot 5 \quad 21 \cdot 15$ $23 \cdot 75 \quad 24 \cdot 95$ $21 \cdot 35 \quad 22 \cdot 45$ $22 \cdot 2 \quad 23 \cdot 4$ $20 \cdot 7 \quad 21 \cdot 85$ $21 \cdot 25 \quad 22 \cdot 4$ $23 \cdot 2 \quad 24 \cdot 4$ $19 \cdot 8 \quad 20 \cdot 85$ $20 \cdot 85 \quad 22 \cdot 05$ $19 \cdot 2 \quad 20 \cdot 3$ 19.65 $\begin{array}{ll}22 \cdot 65 & 23 \cdot 75 \\ 22 \cdot 9 & 24 \cdot 15\end{array}$ $22 \cdot 45 \quad 23 \cdot 7$ $22 \cdot 35 \quad 23 \cdot 7$ $21 \cdot 8 \quad 22.95 \quad 26.7$ $20 \cdot 3 \quad 21 \cdot 5 \quad 24 \cdot$ $23.35 \quad 24 \cdot 65 \quad 27 \cdot 25$ $19 \cdot 2 \quad 20 \cdot 3$ $25 \cdot 6 \quad 25 \cdot 0 \quad 27 \cdot 6$ $22 \cdot 4 \quad 23 \cdot 6$ $20 \cdot 8 \quad 21 \cdot 9$ $21.85 \quad 23 \cdot 0 \quad 25.6$ $21 \cdot 2 \quad 22 \cdot 39 \quad 24 \cdot 95$ $20 \cdot 2 \quad 21 \cdot 3 \quad 23 \cdot 6$ $\begin{array}{lll}20 \cdot 6 & 21 \cdot 7 & 24 \cdot 05\end{array}$

$\begin{array}{ll}27 \cdot 35 & 15 \\ 24 \cdot 15 & 14 \\ 25 \cdot 5 & 15 \\ 23 \cdot 0 & 15 \\ 24 \cdot 95 & 13 \\ 25 \cdot 3 & 15 \\ 28 \cdot 6 & 14 \\ 25 \cdot 75 & 15 \\ 27 \cdot 15 & 15 \\ 25 \cdot 3 & 13 \\ 24 \cdot 5 & 1 \\ 24 \cdot 85 & 15 \\ 23 \cdot 25 & 15 \\ 23 \cdot 7 & 15 \\ 24 \cdot 0 & 9+0 \\ 27 \cdot 6 & 16 \\ 25 \cdot 1 & 16 \\ 25 \cdot 95 & 14 \\ 24 \cdot 4 & 16 \\ 25 \cdot 05 & 15 \\ 27 \cdot 2 & 1+ \\ 23 \cdot 3 & 14 \\ 24 \cdot 55 & 15 \\ 26 \cdot 0 & 15 \\ 22 \cdot 55 & 15 \\ 26 \cdot 45 & 16 \\ 26 \cdot 8 & 15 \\ 26 \cdot 35 & 14 \\ 26 \cdot 15 & 15 \\ 25 \cdot 7 & 16 \\ 24 \cdot 0 & 15 \\ 27 \cdot 25 & 16 \\ 25 \cdot 5 & 15 \\ 22 \cdot 45 & 14 \\ 27 \cdot 6 & 15 \\ 26 \cdot 4 & 15 \\ 24 \cdot 5 & 14 \\ 25 \cdot 6 & 15 \\ 24 \cdot 95 & 14 \\ 23 \cdot 6 & 15 \\ 24 \cdot 05 & 17 \\ & \end{array}$

13
15
14
15
15
13
15
14
15
15
13
14
15
15
15
15
16
16
14
16
5
-14
14
15
15
15
16
15
14
15
16
15
16
15
14
15
15
14
15
14
15
17
17
17
17
17
17
17
17
17
17

$\begin{array}{ll}2+17 & 2+14 \\ 3+17 & 2+15 \\ 3+16 & 2+15 \\ 2+17 & 3+16 \\ 2+17 & 3+15 \\ 2+16 & 2+16 \\ 3+16 & 2+15 \\ 3+16 & 2+15 \\ 2+17 & 2+15 \\ 3+16 & 3+15 \\ 2+16 & 3+15 \\ 2+17 & 3+15 \\ 2+16 & 3+15 \\ 2+17 & 2+17 \\ 2+16 & 2+17 \\ 3+16 & 3+16 \\ 3+16 & 3+15 \\ 2+15 & 2+15 \\ 3+17 & 2+15 \\ 2+16 & 2+15 \\ 2+17 & 2+15 \\ 2+18 & 2+15 \\ 2+17 & 3+14 \\ 3+16 & 2+16 \\ 2+17 & 2+16 \\ 3+15 & 2+16 \\ 2+17 & 2+14 \\ 3+16 & 2+15 \\ 3+17 & 2+15 \\ 2+17 & 2+14 \\ 2+16 & 3+15 \\ 3+16 & 2+15 \\ 2+16 & 2+15 \\ 2+17 & 2+15 \\ 2+17 & 3+16 \\ 2+16 & 2+16 \\ 2+16 & 1+15 \\ 3+17 & 1+15 \\ 2+17 & 2+17 \\ 3+17 & 2+16 \\ 2+17 & 2+16 \\ 2+17 & 2+17 \\ 2+15\end{array}$

$115 \quad 0$ iv-vi $0+32 \quad 56$ $\begin{array}{llll}166 & \mathrm{v} & 3+31 & 56\end{array}$

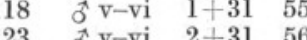
$95 \quad 0$ iv- $\quad 0+33$ 95 .

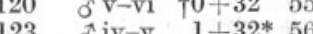

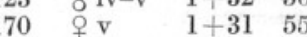
123 ठิ $\quad 2+31 \quad 5$. 170 के $\mathrm{v} \quad 1+31 \quad 5$ $\begin{array}{llll}28 & \text { 우 } v \text {-vi } & 1+31 & 55\end{array}$ $\begin{array}{llll}15 & \text { v-vi } & 0+32 & 56\end{array}$ 92 iv-iv $1+325$ 2 o iv-v $0+32 \quad 55$ Oiv-v $+0+32 \quad 56$ ㅇ iii-iv $2+32 \quad 56$ के $\mathrm{v} \quad 2+31 \quad 56$ ơ $\quad 0+31^{*} 56$ o v $\quad 2+31 \quad 56$ 年 $1+31 *$ $\begin{array}{lll}+\mathrm{vi} & 1+31 & 56 \\ \mathrm{v} & 0+33 & 57\end{array}$ $\begin{array}{lll}+\mathrm{v} & 0+33 & 57 \\ \mathrm{iv}-\mathrm{v} & 1+32 & 55\end{array}$ $\begin{array}{lll}\mathrm{v}-\mathrm{vi} & 1+32 & 55\end{array}$ Oे iv-v $\quad 0+33 \quad 56$ ơ iii-iv $2+31 \quad 56$ Tiv-vi $\quad 2+32 \quad 57$ $\begin{array}{lll}7 \mathrm{v} & 2+32 & 56\end{array}$ O iv-v 1+32* 56 ot v-vi $+0+31 \quad 55$ ô v-vi $2+31 \quad 55$ oิ v $\quad 0+33 \quad 56$ 우 vi $\quad 0+34 \quad 56$

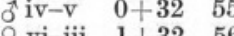
$\begin{array}{lll}1+31 & 55\end{array}$ $0 \mathrm{v}, \quad 1+31 \quad 55$ $\begin{array}{lll}+ \text { v } & 0+32 & 56 \\ \text { 우 iii-iv } & 1+33 & 56\end{array}$ 아 $\mathrm{v} \quad 0+32 \quad 55$

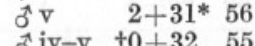
ơ iv-v $+0+32 \quad 56$ 


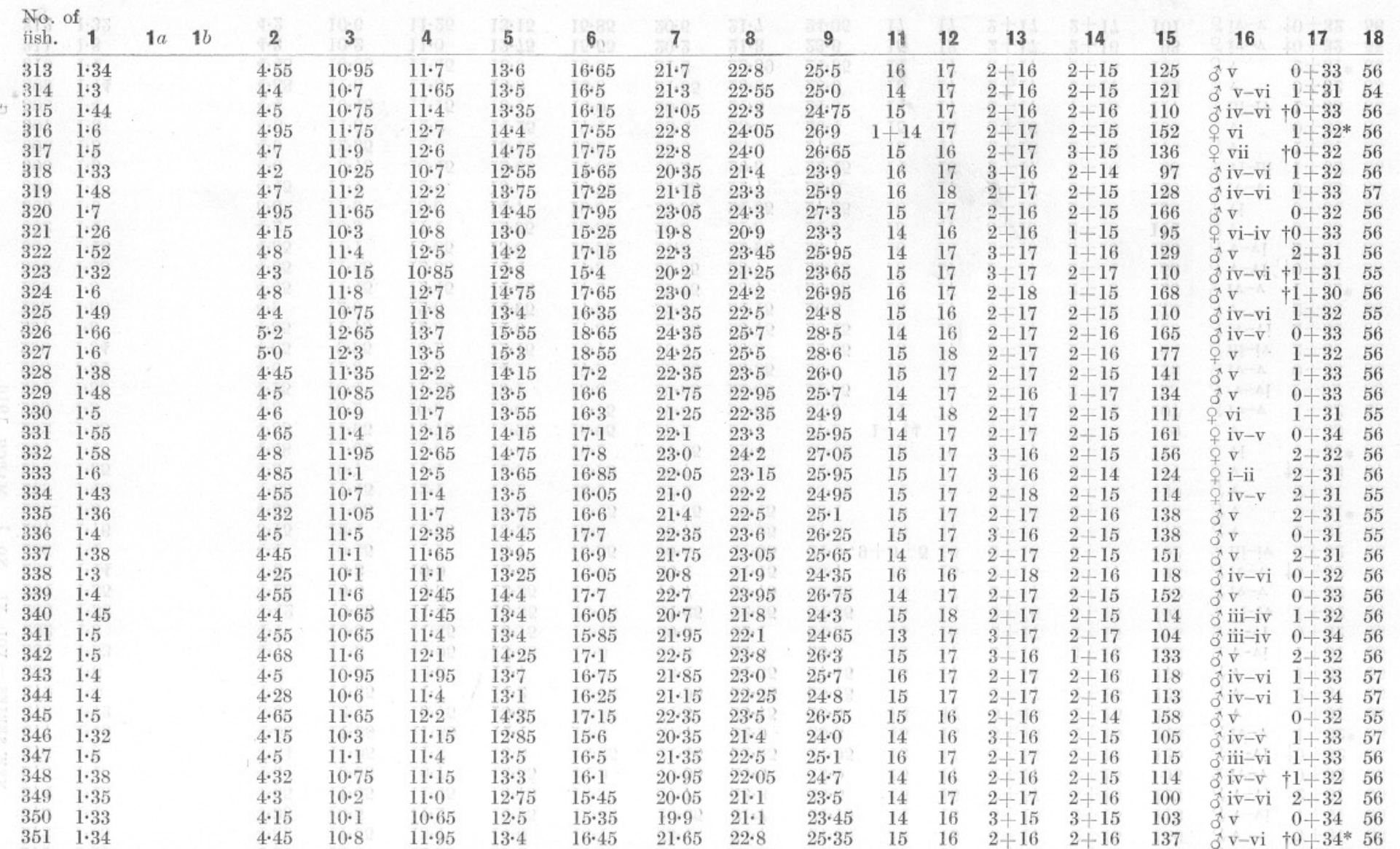




\begin{tabular}{|c|c|c|c|c|c|c|c|c|c|c|c|c|c|c|c|c|c|}
\hline 352 & $1 \cdot 42$ & $4 \cdot 4$ & $10 \cdot 6$ & $11 \cdot 1$ & $13 \cdot 3$ & $16 \cdot 05$ & $20 \cdot 9$ & $22 \cdot 0$ & $24 \cdot 65$ & 15 & 16 & $2+17$ & $2+15$ & 117 & $\sigma^{2} \mathrm{v}$ & $2+31 *$ & 55 \\
\hline 353 & $1 \cdot 38$ & $4 \cdot 25$ & $10 \cdot 8$ & $11 \cdot 75$ & $13 \cdot 55$ & $16 \cdot 7$ & $21 \cdot 75$ & $22 \cdot 9$ & $25 \cdot 35$ & 14 & 17 & $2+15$ & $2+15$ & 128 & of-vi & $2+33$ & 56 \\
\hline 354 & $1 \cdot 5$ & $4 \cdot 95$ & $12 \cdot 2$ & $13 \cdot 4$ & $15 \cdot 2$ & 18.55 & $23 \cdot 8$ & $25 \cdot 0$ & $28 \cdot 15$ & 13 & 16 & $2+17$ & $2+14$ & 160 & $\mathbf{v}$ & $1+31$ & 55 \\
\hline 355 & $1 \cdot 35$ & $4 \cdot 3$ & $10 \cdot 6$ & $11 \cdot 35$ & $13 \cdot 4$ & $16 \cdot 25$ & $20 \cdot 95$ & $22 \cdot 1$ & $24 \cdot 75$ & 16 & 17 & $2+17$ & $2+15$ & 126 & $+\mathrm{iv}-\mathrm{v}$ & $1+30$ & 55 \\
\hline 356 & $1 \cdot 43$ & $4 \cdot 6$ & $11 \cdot 05$ & $11 \cdot 7$ & $13 \cdot 6$ & $16 \cdot 45$ & $21 \cdot 4$ & $22 \cdot 65$ & $25 \cdot 15$ & 14 & 17 & $2+17$ & $2+17$ & 113 & ôiv-vi & $0+33$ & 56 \\
\hline 357 & 1.42 & $4 \cdot 4$ & $10 \cdot 3$ & $11 \cdot 15$ & $12 \cdot 8$ & $15 \cdot 65$ & $20 \cdot 35$ & $21 \cdot 55$ & $23 \cdot 9$ & 15 & 16 & $3+16$ & $3+14$ & 98 & op iv & $1+32$ & 56 \\
\hline 358 & $1 \cdot 46$ & $4 \cdot 45$ & $10 \cdot 8$ & $11 \cdot 5$ & $13 \cdot 45$ & $16 \cdot 3$ & $20 \cdot 8$ & $21 \cdot 85$ & $24 \cdot 2$ & 15 & 17 & $2+17$ & $2+16$ & 109 & iv & $1+31$ & 56 \\
\hline 359 & $1 \cdot 34$ & $4 \cdot 2$ & $9 \cdot 75$ & $10 \cdot 75$ & $12 \cdot 25$ & $14 \cdot 8$ & $19 \cdot 6$ & $20 \cdot 8$ & $22 \cdot 9$ & 14 & 17 & $2+16$ & $3+14$ & 88 & o $\mathrm{iii}-\mathrm{vi}$ & $\uparrow 0+33$ & 56 \\
\hline 360 & 1.53 & $4 \cdot 75$ & $11 \cdot 3$ & $12 \cdot 4$ & $13 \cdot 9$ & $19 \cdot 85$ & $21 \cdot 85$ & 22.95 & $25 \cdot 5$ & 14 & 17 & $2+17$ & $2+15$ & 116 & ơ iii & $1+32$ & 56 \\
\hline 361 & $1 \cdot 27$ & $3 \cdot 95$ & $9 \cdot 6$ & $10 \cdot 25$ & $12 \cdot 0$ & $14 \cdot 25$ & $18 \cdot 83$ & $19 \cdot 8$ & $22 \cdot 15$ & 15 & 16 & $2+16$ & $2+15$ & 77 & iq iv & $1+33$ & 56 \\
\hline 362 & $1 \cdot 55$ & $4 \cdot 85$ & $11 \cdot 95$ & $12 \cdot 6$ & $14 \cdot 8$ & $17 \cdot 65$ & $22 \cdot 75$ & $24 \cdot 0$ & $26 \cdot 65$ & 14 & 16 & $2+17$ & $2+16$ & 147 & ôiv-vi & $1+32$ & 56 \\
\hline 363 & $1 \cdot 3$ & $4 \cdot 2$ & $10 \cdot 35$ & $11 \cdot 05$ & $12 \cdot 75$ & $15 \cdot 4$ & $19 \cdot 95$ & $21 \cdot 05$ & $23 \cdot 45$ & 16 & 16 & $3+16$ & $3+14$ & 84 & o $\mathrm{iii}-\mathrm{iv}$ & $0+32$ & 56 \\
\hline 364 & $1 \cdot 35$ & $4 \cdot 3$ & $10 \cdot 8$ & $11 \cdot 5$ & $13 \cdot 5$ & $16 \cdot 35$ & $21 \cdot 15$ & $22 \cdot 25$ & $24 \cdot 85$ & 15 & 16 & $2+17$ & $2+15$ & 108 & $i v-v$ & $0+34$ & 56 \\
\hline 365 & $1 \cdot 34$ & $4 \cdot 0$ & $9 \cdot 65$ & $10 \cdot 15$ & $11 \cdot 9$ & $14 \cdot 65$ & 18.95 & $20 \cdot 0$ & $22 \cdot 45$ & 15 & 17 & $2+16$ & $2+14$ & 86 & ii-iii & $\dagger 0+33$ & 56 \\
\hline 366 & 1.5 & $4 \cdot 8$ & $11 \cdot 65$ & $12 \cdot 7$ & $14 \cdot 5$ & $17 \cdot 7$ & $22 \cdot 7$ & $23 \cdot 9$ & $26 \cdot 7$ & 14 & 17 & $2+17$ & $2+16$ & 156 & i v & $2+31$ & 56 \\
\hline 367 & $1 \cdot 33$ & $4 \cdot 2$ & $9 \cdot 8$ & $11 \cdot 1$ & $12 \cdot 5$ & $15 \cdot 0$ & $19 \cdot 7$ & $20 \cdot 6$ & $22 \cdot 6$ * & 14 & 15 & $2+17$ & $2+14$ & 90 & ô iv-vi & $2+31$ & 56 \\
\hline 368 & $1 \cdot 45$ & $4 \cdot 35$ & $9 \cdot 95$ & $10 \cdot 75$ & $12 \cdot 2$ & $15 \cdot 1$ & $19 \cdot 5$ & $20 \cdot 55$ & $22 \cdot 8$ & 15 & 17 & $2+15$ & $2+15$ & 88 & ô iii-iv & $3+31$ & 56 \\
\hline 369 & $1 \cdot 3$ & $4 \cdot 2$ & $9 \cdot 9$ & $10 \cdot 7$ & $12 \cdot 6$ & $15 \cdot 45$ & $20 \cdot 1$ & $21 \cdot 15$ & $23 \cdot 8$ & 15 & 17 & $2+17$ & $2+16$ & 108 & ờ iv-vi & $1+32$ & 55 \\
\hline 370 & $1 \cdot 4$ & 4.53 & $11 \cdot 1$ & $12 \cdot 2$ & $13 \cdot 75$ & $17 \cdot 15$ & $22 \cdot 05$ & $23 \cdot 2$ & $25 \cdot 9$ & 16 & 17 & $2+17$ & $2+14$ & 131 & $\mathrm{O}^{1} \mathrm{v}$ & $1+32$ & 56 \\
\hline 371 & $1 \cdot 45$ & $4 \cdot 73$ & $11 \cdot 4$ & $12 \cdot 45$ & $14 \cdot 0$ & $17 \cdot 25$ & $22 \cdot 25$ & $23 \cdot 45$ & $26 \cdot 0$ & 15 & 16 & $2+17$ & $2+15$ & 117 & 우 iii-iv & $0+31$ & 56 \\
\hline 372 & $1 \cdot 46$ & $4 \cdot 6$ & $11 \cdot 4$ & $12 \cdot 4$ & $14 \cdot 1$ & $17 \cdot 0$ & $22 \cdot 1$ & $23 \cdot 25$ & $25 \cdot 85$ & 14 & 17 & $2+16$ & $3+14$ & 127 & o iv-v & $0+33$ & 55 \\
\hline 373 & $1 \cdot 3$ & $4 \cdot 23$ & $10 \cdot 15$ & $11 \cdot 0$ & $12 \cdot 85$ & $15 \cdot 35$ & $20 \cdot 45$ & $21 \cdot 55$ & $24 \cdot 15$ & 14 & 17 & $2+17$ & $2+17$ & 102 & o iii-iv & $1+32$ & 56 \\
\hline 374 & $1 \cdot 4$ & $4 \cdot 4$ & 10.7 & $11 \cdot 25$ & $13 \cdot 5$ & $15 \cdot 85$ & $20 \cdot 6$ & $21 \cdot 75$ & $24 \cdot 15$ & 15 & 17 & $2+16$ & $3+14$ & 106 & ôv & $1+32$ & 56 \\
\hline 375 & $1 \cdot 36$ & $4 \cdot 53$ & $11 \cdot 05$ & 11.75 & 13.95 & $16 \cdot 55$ & $21 \cdot 7$ & $22 \cdot 85$ & $25 \cdot 15$ & 15 & 16 & $2+17$ & $3+16$ & 120 & $0 \mathrm{~V}$ & $1+33$ & 56 \\
\hline 376 & $1 \cdot 42$ & $4 \cdot 4$ & $10 \cdot 65$ & $11 \cdot 35$ & $13 \cdot 35$ & $15 \cdot 7$ & $20 \cdot 3$ & $21 \cdot 5$ & $24 \cdot 0$ & 15 & 17 & $2+17$ & $2+17$ & 112 & $i v-v$ & $2+30$ & 55 \\
\hline 377 & $1 \cdot 43$ & $4 \cdot 6$ & 11.75 & 12.5 & $14 \cdot 5$ & $17 \cdot 4$ & $22 \cdot 3$ & $23 \cdot 45$ & $26 \cdot 15$. & 15 & 16 & $2+15$ & $2+15$ & 145 & iv $-\mathrm{v}$ & $0+32$ & 55 \\
\hline 378 & $1 \cdot 4$ & $4 \cdot 66$ & 11.45 & 12.55 & 13.95 & $17 \cdot 35$ & $22 \cdot 6$ & 23.8 & $26 \cdot 55$ & 15 & 17 & $3+15$ & $2+14$ & 141 & के $\mathbf{v}$ & $0+33$ & 56 \\
\hline 379 & $1 \cdot 3$ & $4 \cdot 16$ & 10.05 & $10 \cdot 8$ & $12 \cdot 35$ & $15 \cdot 0$ & $19 \cdot 35$ & $25 \cdot 0$ & $22 \cdot 75$ & 15 & 16 & $3+16$ & $2+15$ & 95 & ô iv-vi & $1+33$ & 57 \\
\hline 380 & $1 \cdot 4$ & $4 \cdot 3$ & $10 \cdot 7$ & $11 \cdot 4$ & $13 \cdot 25$ & $16 \cdot 05$ & $21 \cdot 0$ & $22 \cdot 1$ & $24 \cdot 6$ & 14 & 17 & $2+16$ & $2+15$ & 112 & ôv-vi & $1+32$ & 55 \\
\hline 81 & $1 \cdot 32$ & $4 \cdot 16$ & $10 \cdot 05$ & $10 \cdot 55$ & $12 \cdot 3$ & $14 \cdot 8$ & $19 \cdot 3$ & $20 \cdot 3$ & $22 \cdot 6$ & 15 & 17 & $2+15$ & $2+14$ & 85 & ô iv & $2+32$ & 56 \\
\hline 82 & $1 \cdot 4$ & $4 \cdot 3$ & $10 \cdot 45$ & $11 \cdot 45$ & $13 \cdot 15$ & $16 \cdot 05$ & $20 \cdot 7$ & $21 \cdot 8$ & $24 \cdot 15$ & 15 & 18 & $2+17$ & $3+15$ & 106 & 우 iii-iv & $0+32$ & 56 \\
\hline 383 & $1 \cdot 46$ & $4 \cdot 6$ & 11.45 & 12.05 & 13.9 & $17 \cdot 0$ & $21 \cdot 8$ & 22.95 & $25 \cdot 45$ & 15 & 16 & $2+17$ & $2+17$ & 113 & $\hat{o}$ iv & $1+31$ & 56 \\
\hline 84 & $1 \cdot 35$ & $4 \cdot 06$ & $9 \cdot 8$ & $10 \cdot 9$ & $12 \cdot 1$ & $15 \cdot 0$ & $19 \cdot 55$ & $20 \cdot 7$ & $23 \cdot 1$ & 14 & 17 & $2+16$ & $2+15$ & 87 & ô iv & $0+33$ & 56 \\
\hline 85 & $1 \cdot 37$ & $4 \cdot 45$ & $10 \cdot 55$ & $11 \cdot 4$ & $13 \cdot 2$ & $15 \cdot 8$ & $20 \cdot 65$ & $21 \cdot 75$ & $24 \cdot 2$ & 15 & 17 & $2+18$ & $2+16$ & 104 & $0 \hat{\mathrm{V}}$ & $1+32$ & 56 \\
\hline 86 & $1 \cdot 4$ & $4 \cdot 33$ & $10 \cdot 05$ & $10 \cdot 9$ & $12 \cdot 6$ & $15 \cdot 3$ & $20 \cdot 0$ & $21 \cdot 05$ & $23 \cdot 5$ & 15 & 17 & $2+16$ & $2+15$ & 91 & 우 iii-iv & $1+32$ & 56 \\
\hline 387 & $1 \cdot 47$ & $4 \cdot 55$ & $11 \cdot 25$ & $11 \cdot 8$ & $13 \cdot 8$ & 16.5 & $21 \cdot 7$ & $22 \cdot 9$ & $25 \cdot 65$ & 14 & 17 & $2+16$ & $2+16$ & 136 & v $\mathrm{v}$ & $1+32$ & 56 \\
\hline 88 & 1.53 & $4 \cdot 62$ & $12 \cdot 15$ & $12 \cdot 7$ & $14 \cdot 75$ & $17 \cdot 9$ & 22.85 & $24 \cdot 1$ & $26 \cdot 8$ & 15 & 16 & $3+16$ & $2+15$ & 138 & $\hat{o} \mathrm{~V}$ & $0+32$ & 56 \\
\hline 89 & $1 \cdot 44$ & $4 \cdot 4$ & $10 \cdot 6$ & $11 \cdot 45$ & $13 \cdot 2$ & $15 \cdot 85$ & $20 \cdot 75$ & $21 \cdot 85$ & $24 \cdot 3$ & 13 & 17 & $2+16$ & $2+16$ & 110 & iv-v & $0+32$ & 56 \\
\hline 390 & $1 \cdot 34$ & $4 \cdot 1$ & $10 \cdot 35$ & $11 \cdot 0$ & $13 \cdot 1$ & $15 \cdot 95$ & $20 \cdot 9$ & $22 \cdot 05$ & $24 \cdot 4$ & 15 & 16 & $2+17$ & $2+16$ & 124 & $\partial v$ & $1+32$ & 56 \\
\hline 391 & $1 \cdot 4$ & $4 \cdot 4$ & $10 \cdot 9$ & $12 \cdot 3$ & $13 \cdot 8$ & $17 \cdot 35$ & $22 \cdot 15$ & $23 \cdot 3$ & $25 \cdot 9$ & 15 & 16 & $2+16$ & $2+16$ & 147 & $\delta \mathrm{v}-\mathrm{vi}$ & $0+32$ & 56 \\
\hline & $1 \cdot 3$ & $4 \cdot 1$ & $9 \cdot 95$ & $10 \cdot 4$ & $12 \cdot 45$ & $14 \cdot 5$ & $19 \cdot 2$ & $20 \cdot 35$ & $22 \cdot 75$ & 14 & 15 & $2+17$ & $2+15$ & 90 & $\partial^{2} \mathrm{v}-\mathrm{vi}$ & $1+32$ & 55 \\
\hline 93 & $1 \cdot 64$ & $4 \cdot 9$ & $12 \cdot 3$ & $12 \cdot 9$ & $15 \cdot 25$ & $18 \cdot 3$ & $23 \cdot 3$ & $24 \cdot 55$ & $27 \cdot 3$ & 16 & 17 & $3+17$ & $3+15$ & 164 & $\widehat{o} \mathrm{~V}$ & $1+32$ & 57 \\
\hline
\end{tabular}


No, of

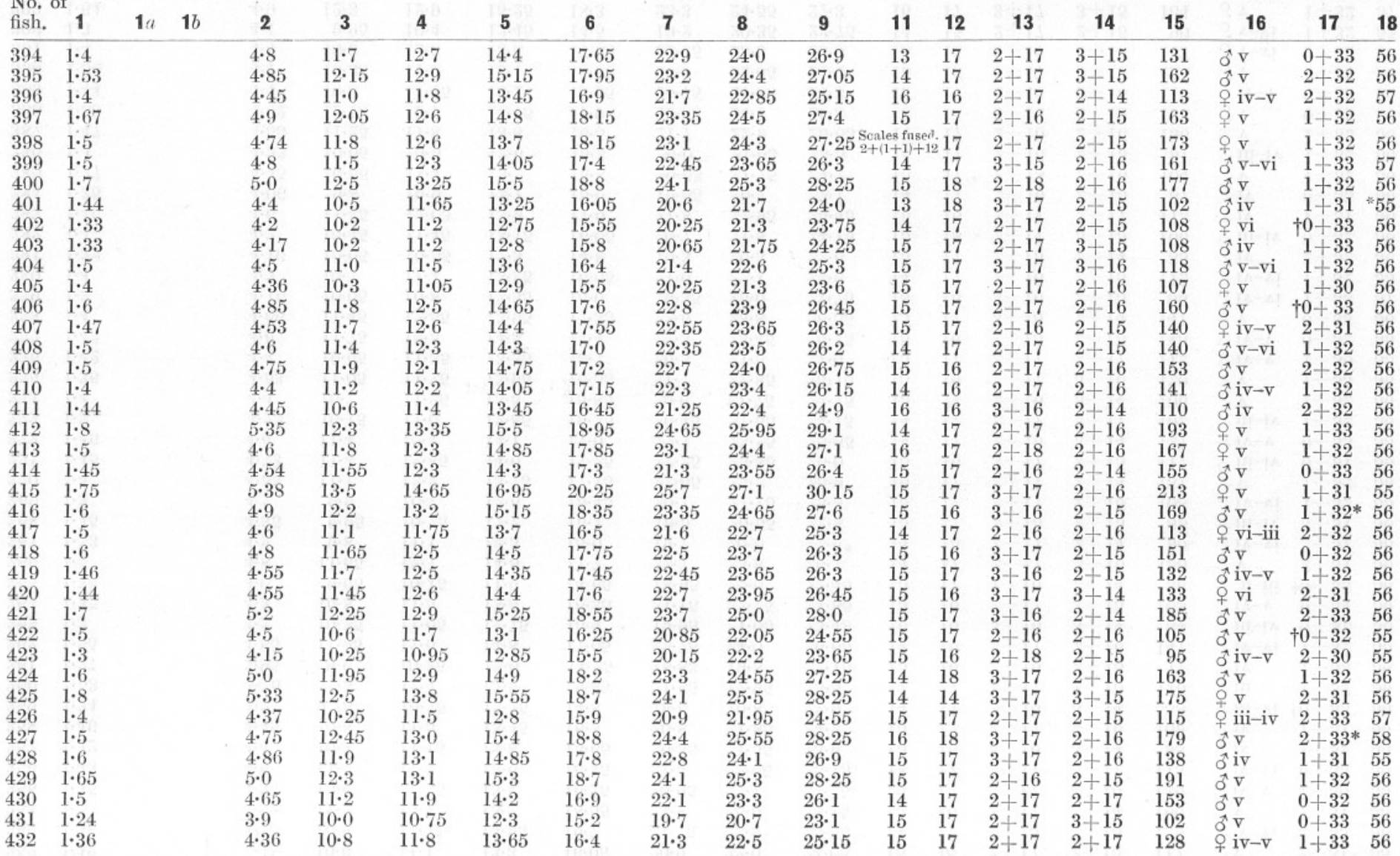




\begin{tabular}{|c|c|c|c|c|c|c|c|c|c|c|c|c|c|c|c|c|c|c|c|c|}
\hline fish. & 1 & $1 a$ & $1 b$ & 2 & 3 & 4 & 5 & 6 & 7 & 8 & 9 & 11 & 12 & 13 & 14. & 15 & 16 & 17 & 18 & \\
\hline 474 & $1 \cdot 3$ & & & $4 \cdot 3$ & $10 \cdot 15$ & $10 \cdot 55$ & $12 \cdot 75$ & $14 \cdot 9$ & $19 \cdot 6$ & $20 \cdot 8$ & $23 \cdot 3$ & 14 & 16 & $2+18$ & $2+16$ & 103 & 우 iv & $0+32$ & 55 & \\
\hline 475 & 1.5 & & & $4 \cdot 63$ & $11 \cdot 5$ & $12 \cdot 6$ & $14 \cdot 25$ & 17.85 & $22 \cdot 6$ & $23 \cdot 85$ & $26 \cdot 6$ & 15 & 16 & $3+16$ & $2+14$ & 159 & $\mathrm{v}$ & $1+32$ & 56 & \\
\hline 476 & 1.35 & & & $4 \cdot 25$ & $10 \cdot 65$ & $11 \cdot 45$ & $13 \cdot 25$ & $16 \cdot 0$ & $21 \cdot 0$ & $22 \cdot 1$ & $24 \cdot 5$ & 14 & 16 & $3+17$ & $2+15$ & 116 & $\sigma^{t} \mathrm{v}-\mathrm{vi}$ & $0+32$ & 56 & \\
\hline 477 & 1.7 & & & $5 \cdot 1$ & $12 \cdot 65$ & $13 \cdot 7$ & $15 \cdot 55$ & $19 \cdot 1$ & $24 \cdot 6$ & $25 \cdot 8$ & $28 \cdot 7$ & 14 & 17 & $3+16$ & $2+15$ & 155 & iv & $1+32$ & 56 & \\
\hline 478 & 1.5 & & & $4 \cdot 68$ & $11 \cdot 3$ & $12 \cdot 2$ & 13.95 & $17 \cdot 45$ & $22 \cdot 05$ & $23 \cdot 3$ & $26 \cdot 15$ & 16 & 16 & $3+16$ & $2+14$ & 141 & o $v$-vi & $1+31$ & 55 & \\
\hline 479 & $1 \cdot 3$ & & & $4 \cdot 0$ & $9 \cdot 75$ & $11 \cdot 0$ & $12 \cdot 45$ & $15 \cdot 1$ & $19 \cdot 75$ & $20 \cdot 85$ & 23.05 & 15 & 17 & $2+17$ & $2+15$ & 94 & ii-iii & $2+31$ & 56 & \\
\hline 480 & $1 \cdot 6$ & & & $4 \cdot 9$ & $11 \cdot 6$ & $12 \cdot 2$ & $14 \cdot 4$ & $17 \cdot 05$ & $22 \cdot 15$ & $23 \cdot 3$ & $26 \cdot 1$ & 15 & 17 & $3+17$ & $2+14$ & 147 & $\mathrm{v}$ & $2+31$ & 56 & \\
\hline 481 & $1 \cdot 4$ & & & $4 \cdot 32$ & $10 \cdot 3$ & $10 \cdot 8$ & $12 \cdot 45$ & $15 \cdot 5$ & $19 \cdot 6$ & $20 \cdot 7$ & $23 \cdot 15$ & 14 & 17 & $2+17$ & $2+14$ & 99 & ㅇi-iii & $0+33$ & 56 & \\
\hline 482 & $1 \cdot 28$ & & & $3 \cdot 9$ & 9.55 & $9 \cdot 85$ & 11.85 & 13.95 & $18 \cdot 35$ & $19 \cdot 5$ & $21 \cdot 7$ & 16 & 17 & $3+16$ & $2+15$ & 84 & $\mathrm{v}$ & $1+31$ & 55 & \\
\hline 483 & $1 \cdot 53$ & & & $4 \cdot 9$ & $12 \cdot 35$ & 12.9 & $15 \cdot 6$ & 18.65 & $23 \cdot 9$ & $25 \cdot 3$ & $28 \cdot 2$ & 14 & 18 & $3+18$ & $2+16$ & 185 & V & $1+33$ & 56 & \\
\hline 484 & $1 \cdot 6$ & & & $4 \cdot 7$ & $11 \cdot 45$ & $12 \cdot 15$ & $14 \cdot 2$ & $17 \cdot 4$ & $22 \cdot 3$ & $23 \cdot 5$ & $26 \cdot 05$ & 15 & 16 & $3+16$ & $1+16$ & 146 & v & $1+34$ & 57 & \\
\hline 485 & $1 \cdot 38$ & & & $4 \cdot 35$ & $10 \cdot 5$ & $11 \cdot 15$ & $13 \cdot 2$ & $15 \cdot 6$ & $20 \cdot 75$ & $21 \cdot 8$ & $24 \cdot 45$ & 13 & 17 & $3+18$ & $2+16$ & 102 & 우 iii-iv & $0+33$ & 57 & \\
\hline 486 & 1.45 & & & $4 \cdot 44$ & $10 \cdot 7$ & $11 \cdot 6$ & 13.55 & $16 \cdot 7$ & $21 \cdot 4$ & $22 \cdot 7$ & $25 \cdot 25$ & 15 & 17 & $2+18$ & $2+15$ & 137 & $\delta \mathrm{v}$ & $1+30$ & 55 & \\
\hline 487 & 1.5 & & & $4 \cdot 42$ & 10.8 & 11.5 & 13.5 & $16 \cdot 7$ & $21 \cdot 3$ & $22 \cdot 5$ & $25 \cdot 1$ & 15 & 17 & $2+17$ & $1+14$ & 125 & iv & $0+32$ & 56 & \\
\hline 488 & 1.7 & & & $5 \cdot 14$ & $12 \cdot 36$ & $13 \cdot 3$ & $15 \cdot 7$ & $18 \cdot 55$ & $23 \cdot 8$ & $25 \cdot 1$ & $28 \cdot 0$ & 16 & 17 & $3+18$ & $2+16$ & 190 & & $1+33$ & 56 & \\
\hline 489 & $1 \cdot 56$ & & & 4.62 & $11 \cdot 35$ & 11.85 & $14 \cdot 1$ & $16 \cdot 65$ & $21 \cdot 9$ & $23 \cdot 0$ & $25 \cdot 6$ & 15 & 17 & $3+17$ & $2+15$ & 126 & iv-v & $2+32$ & 56 & \\
\hline 490 & 1.76 & & & $4 \cdot 96$ & $12 \cdot 4$ & $13 \cdot 4$ & $15 \cdot 5$ & 18.55 & 23.95 & $25 \cdot 3$ & $28 \cdot 0$ & 13 & 18 & $2+17$ & $2+15$ & 187 & . & $0+32$ & 55 & \\
\hline 491 & $1 \cdot 36$ & & & $4 \cdot 2$ & $10 \cdot 25$ & $11 \cdot 1$ & $13 \cdot 0$ & $15 \cdot 75$ & $20 \cdot 6$ & $21 \cdot 75$ & $24 \cdot 15$ & $1+0+12$ & 16 & $2+17$ & $2+15$ & 103 & & $1+32$ & 56 & \\
\hline 492 & $1 \cdot 6$ & & & $4 \cdot 92$ & $12 \cdot 0$ & $12 \cdot 15$ & $14 \cdot 7$ & $17 \cdot 6$ & 22.55 & $23 \cdot 75$ & $26 \cdot 3$ & 15 & 17 & $3+16$ & $1+17$ & 159 & & $2+31$ & 56 & \\
\hline 493 & 1.5 & & & $4 \cdot 36$ & 10.75 & $11 \cdot 6$ & $13 \cdot 45$ & $16 \cdot 45$ & $21 \cdot 35$ & $22 \cdot 5$ & $25 \cdot 2$ & 16 & 17 & $3+16$ & $2+15$ & 133 & 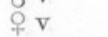 & $0+32$ & 56 & \\
\hline 494 & 1.5 & & & $4 \cdot 5$ & $11 \cdot 0$ & $12 \cdot 0$ & 13.55 & $16 \cdot 4$ & $21 \cdot 45$ & $22 \cdot 65$ & $25 \cdot 3$ & 15 & 15 & $3+16$ & & 127 & iv-v & $1+31$ & 56 & \\
\hline 495 & 1.6 & & & $5 \cdot 24$ & $12 \cdot 75$ & $13 \cdot 9$ & $15 \cdot 95$ & $18 \cdot 8$ & $24 \cdot 6$ & $25 \cdot 95$ & $28 \cdot 65$ & 14 & 16 & $3+17$ & $2+15$ & 184 & & $2+30$ & 55 & \\
\hline 496 & 1.7 & & & $5 \cdot 03$ & $12 \cdot 3$ & $13 \cdot 75$ & $15 \cdot 25$ & $18 \cdot 4$ & $23 \cdot 7$ & $25 \cdot 05$ & $27 \cdot 8$ & 14 & 15 & $3+15$ & $2+15$ & 163 & 0 & $0+33$ & 56 & \\
\hline 497 & $1 \cdot 76$ & & & $5 \cdot 42$ & $13 \cdot 1$ & 13.6 & $16 \cdot 05$ & $18 \cdot 8$ & $24 \cdot 35$ & $25 \cdot 7$ & 28.65 & 15 & 17 & $2+17$ & $2+16$ & 191 & & $1+32$ & 56 & \\
\hline 498 & $1 \cdot 7$ & & & $5 \cdot 0$ & $11 \cdot 8$ & 12.8 & $14 \cdot 5$ & $17 \cdot 7$ & $22 \cdot 8$ & $24 \cdot 15$ & $27 \cdot 1$ & 15 & 18 & $2+16$ & $1+15$ & 154 & & $1+32$ & 55 & \\
\hline 499 & $1 \cdot 4$ & & & $4 \cdot 5$ & $10 \cdot 85$ & $12 \cdot 0$ & $13 \cdot 9$ & $16 \cdot 6$ & $21 \cdot 85$ & $23 \cdot 0$ & $25 \cdot 7$ & 14 & 17 & $2+18$ & $2+15$ & 133 & $\mathrm{iv}-\mathrm{v}$ & $0+32$ & 56 & \\
\hline 500 & 1.58 & & & $4 \cdot 85$ & $11 \cdot 8$ & $12 \cdot 8$ & $14 \cdot 7$ & $18 \cdot 1$ & $22 \cdot 75$ & $24 \cdot 0$ & $26 \cdot 65$ & 16 & 17 & $2+17$ & $2+14$ & 158 & . & $0+33$ & 55 & \\
\hline 501 & 1.58 & & & $4 \cdot 72$ & 11.95 & $13 \cdot 0$ & $14 \cdot 9$ & $17 \cdot 9$ & $23 \cdot 3$ & $24 \cdot 55$ & $27 \cdot 45$ & 15 & 18 & $2+17$ & $2+16$ & 175 & & $2+32$ & 57 & \\
\hline 502 & 1.58 & & & $4 \cdot 75$ & $11 \cdot 1$ & 12.05 & $13 \cdot 9$ & $16 \cdot 8$ & $21 \cdot 75$ & $22 \cdot 9$ & $25 \cdot 6$ & 15 & 16 & $3+17$ & $2+16$ & 131 & $\mathrm{o}^{2} \mathrm{~V}$ & $2+31$ & 56 & \\
\hline 503 & $1 \cdot 6$ & & & $4 \cdot 62$ & $11 \cdot 6$ & $12 \cdot 1$ & $14 \cdot 2$ & $17 \cdot 35$ & $22 \cdot 35$ & $23 \cdot 6$ & $26 \cdot 25$ & 14 & 17 & $3+16$ & $3+15$ & 136 & o v-vi & $0+32$ & 55 & \\
\hline 504 & $1 \cdot 42$ & & & $4 \cdot 3$ & $10 \cdot 85$ & $11 \cdot 65$ & $13 \cdot 75$ & $16 \cdot 4$ & $21 \cdot 65$ & $22 \cdot 75$ & $25 \cdot 2$ & 15 & 17 & $2+17$ & $2+15$. & 121 & iv-v & $0+32$ & 56 & \\
\hline 505 & $1 \cdot 6$ & & & $4 \cdot 65$ & $11 \cdot 3$ & $12 \cdot 0$ & $14 \cdot 15$ & $16 \cdot 8$ & $22 \cdot 5$ & $23 \cdot 6$ & $26 \cdot 25$ & 15 & 17 & $3+17$ & $2+17$ & 136 & o $\mathbf{v}$ & $0+32$ & 56 & \\
\hline 506 & $1 \cdot 36$ & & & $4 \cdot 3$ & $10 \cdot 65$ & $11 \cdot 3$ & $13 \cdot 7$ & $16 \cdot 4$ & $21 \cdot 15$ & $22 \cdot 3$ & $24 \cdot 95$ & 15 & 16 & $3+18$ & $2+15$ & 137 & o $v$-vi & $1+31$ & 55 & \\
\hline 507 & $1 \cdot 65$ & & & $5 \cdot 18$ & $12 \cdot 65$ & $12 \cdot 4$ & $15^{\cdot} 6$ & $18 \cdot 5$ & $23 \cdot 65$ & $24 \cdot 9$ & $27 \cdot 85$ & 15 & 17 & $2+16$ & $2+14$ & 164 & & $1+32$ & 55 & \\
\hline 508 & 1.45 & & & $4 \cdot 4$ & $10 \cdot 15$ & $10 \cdot 85$ & $12 \cdot 7$ & $15 \cdot 0$ & $19 \cdot 95$ & $21 \cdot 05$ & 23.5 & 14 & 17 & $2+16$ & $2+$ & 109 & $\mathrm{~V}$ & $1+32$ & 56 & \\
\hline 509 & $1 \cdot 6$ & & & $4 \cdot 7$ & $10 \cdot 9$ & 11.55 & $13 \cdot 65$ & $16 \cdot 5$ & $21 \cdot 3$ & 22.5 & $25 \cdot 1$ & 15 & 16 & $3+17$ & $2+14$ & 117 & iv-vi & $0+32$ & 56 & \\
\hline 510 & 1.48 & & & $4 \cdot 55$ & $10 \cdot 8$ & $11 \cdot 55$ & $13 \cdot 2$ & $16 \cdot 25$ & $21 \cdot 0$ & $22 \cdot 1$ & $24 \cdot 7$ & 16 & 16 & $2+15$ & $2+15$ & 111 & iii-iv & $0+31$ & 56 & \\
\hline 511 & $1 \cdot 3$ & & & $4 \cdot 2$ & $10 \cdot 35$ & $11 \cdot 1$ & $12 \cdot 8$ & $15 \cdot 65$ & $20 \cdot 3$ & $21 \cdot 4$ & $23 \cdot 9$ & 14 & 17 & $3+16$ & $2+15$ & 113 & $\hat{\mathbf{v}}$ & $1+32$ & 56 & \\
\hline 512 & 1.45 & & & $4 \cdot 38$ & $10 \cdot 4$ & $11 \cdot 1$ & $12 \cdot 7$ & $15 \cdot 7$ & $20 \cdot 4$ & $21 \cdot 5$ & $24 \cdot 0$ & 14 & 17 & $3+16$ & $2+14$ & 106 & o v & $3+30$ & 55 & \\
\hline
\end{tabular}


HaUl.

\section{Herring Race Investigations.}

Position - . Start Pt. E.N.E. about 8 miles to N.N.E. about 3 miles, Date · Jan. 6/15, Landed 10.0 a.m.

Vessel

"G.M.V.," Lowestoft (Steam drifter).

Number examined

Quantity of fish caught

525 .

56 cran (about 50,500)

Jan. 6/15.

Drift-herring.

Examined by

J. H. Orton and others.

The characters are briefly : Length in centimetres from snout to (1) eye, (2) operculum edge, (3) front of dorsal fin, (4) pelvic, (5) back of dorsal, (6) front of anal, (7) root of tail, (8) end of mid-caudal, (9) end of longest caudal ray. Number of

(11) keeled scales (pelvics to anus), (12) rays in pectoral, (13) rays in dorsal, (14) rays in anal, (15) weight in grams, (16)

\begin{tabular}{|c|c|c|c|c|c|c|c|c|c|c|c|c|c|c|c|c|c|}
\hline fish. & 1 & 2 & 3 & 4 & 5 & 6 & 7 & 8 & 9 & 11 & 12 & 13 & 14 & 15 & 16 & 17 & 18 \\
\hline 551 & $1 \cdot 7$ & $5 \cdot 26$ & $12 \cdot 65$ & $14 \cdot 05$ & $15 \cdot 8$ & $19 \cdot 0$ & $24 \cdot 6$ & $25 \cdot 9$ & $28 \cdot 8$ & 15 & 17 & $3+16$ & $3+14$ & 157 & o iv-v & $2+31$ & 55 \\
\hline 552 & 1.55 & $4 \cdot 85$ & $11 \cdot 85$ & $12 \cdot 5$ & $14 \cdot 5$ & $17 \cdot 85$ & $22 \cdot 7$ & $24 \cdot 0$ & $26 \cdot 5$ & 15 & 16 & $2+16$ & $3+15$ & 156 & & & 56 \\
\hline 553 & $1 \cdot 7$ & $5 \cdot 2$ & $12 \cdot 45$ & $13 \cdot 45$ & $15 \cdot 6$ & $19 \cdot 45$ & $24 \cdot 5$ & $25 \cdot 9$ & $28 \cdot 75$ & 16 & 16 & $3+16$ & $3+14$ & 140 & o vii & $2+32$ & 6 \\
\hline 554 & $1 \cdot 6$ & $4 \cdot 8$ & $11 \cdot 8$ & $12 \cdot 55$ & $14 \cdot 6$ & $17 \cdot 65$ & $22 \cdot 75$ & $24 \cdot 0$ & $26 \cdot 9$ & 15 & 16 & $3+16$ & $1+3+13$ & 154 & 오 vi & $2+32$ & 55 \\
\hline 555 & 1.5 & $4 \cdot 85$ & 12 . & $13 \cdot 0$ & $15 \cdot 1$ & $18 \cdot 15$ & $23 \cdot 65$ & $24 \cdot 85$ & $27 \cdot 7$ & 16 & 17 & $3+17$ & $3+15$ & 172 & 우 $\mathrm{i}$ & $1+33$ & 6 \\
\hline 556 & 1.8 & $5 \cdot 4$ & 12.95 & $13 \cdot 7$ & $16 \cdot 1$ & $18 \cdot 85$ & $24 \cdot 6$ & $26 \cdot 0$ & $28 \cdot 75$ & $15_{12}$ & $\begin{array}{l}\text { Fused? } \\
+(1+1)\end{array}$ & $2+17$ & $1+3+14$ & 171 & 우 vi & $1+32$ & 56 \\
\hline 557 & $1 \cdot 4$ & $4 \cdot 4$ & $10 \cdot 8$ & 11.5 & $13 \cdot 45$ & $16 \cdot 05$ & $20 \cdot 9$ & $22 \cdot 1$ & $24 \cdot 45$ & 15 & $\begin{array}{c}(1+1) \\
17\end{array}$ & $3+17$ & $3+15$ & 117 & o iv-v & $2+31$ & 55 \\
\hline 558 & 1.5 & $4 \cdot 85$ & $12 \cdot 05$ & $12 \cdot 65$ & $14 \cdot 9$ & $18 \cdot 0$ & $23 \cdot 1$ & $24 \cdot 2$ & $26 \cdot 9$ & 17 & 18 & & & 149 & o iv-v & $2+$ & 57 \\
\hline 559 & $1 \cdot 6$ & $4 \cdot 85$ & $11 \cdot 3$ & $12 \cdot 6$ & $14 \cdot 35$ & $17 \cdot 6$ & $22 \cdot 3$ & $23 \cdot 5$ & $26 \cdot 3$ & 14 & 17 & $3+$ & $3+$ & 161 & 우 vi & $+0+32$ & 55 \\
\hline 560 & 1.57 & $4 \cdot 8$ & $12 \cdot 1$ & $12 \cdot 7$ & $14 \cdot 8$ & $18 \cdot 15$ & $23 \cdot 4$ & $24 \cdot 7$ & $27 \cdot 2$ & 14 & 16 & 3 & $4-$ & 167 & $\hat{\sigma}$ & $1+33$ & 57 \\
\hline 561 & $1 \cdot 46$ & $4 \cdot 6$ & $11 \cdot 5$ & $12 \cdot 15$ & $14 \cdot 4$ & $17 \cdot 1$ & $22 \cdot 2$ & $23 \cdot 5$ & $26 \cdot 15$ & 14 & 17 & $3+17$ & $3+$ & 162 & $\mathrm{or}^{\circ}$ & $2+31$ & 56 \\
\hline 562 & $1 \cdot 47$ & $4 \cdot 65$ & $11 \cdot 4$ & $12 \cdot 2$ & $14 \cdot 3$ & $17 \cdot 3$ & $22 \cdot 4$ & $23 \cdot 65$ & $26 \cdot 25^{*}$ & 15 & 17 & $3+16$ & $3+$ & 131 & $\hat{\sigma} \mathbf{v}-v i$ & $0+34$ & 57 \\
\hline 563 & $1 \cdot 28$ & $4 \cdot 35$ & $10 \cdot 9$ & $11 \cdot 6$ & $13 \cdot 6$ & 16 . & $21 \cdot 3$ & $22 \cdot 45$ & $25 \cdot 05$ & 15 & 17 & $3-$ & $3+$ & 129 & 우 vi & $\dagger 1+$ & 56 \\
\hline 564 & $1 \cdot 5$ & $4 \cdot 9$ & $12 \cdot 5$ & $13 \cdot 2$ & $15 \cdot 55$ & $18 \cdot 5$ & $23 \cdot 45$ & $24 \cdot 65$ & $27 \cdot 5$ & 14 & 17 & $3+16$ & $3+$ & 145 & ơ vii & $1+31$ & 5 \\
\hline 565 & $1 \cdot 38$ & $4 \cdot 4$ & $11 \cdot 15$ & $11 \cdot 9$ & $13 \cdot 65$ & $16 \cdot 4$ & $21 \cdot 3$ & $22 \cdot 5$ & $25 \cdot 05$ & 14 & 18 & $3+$ & $3+15$ & 121 & ô vi-iii & $1+31$ & 55 \\
\hline 566 & 1.5 & $4 \cdot 45$ & $10 \cdot 75$ & $11 \cdot 4$ & $13 \cdot 34$ & 15.95 & $20 \cdot 65$ & $21 \cdot 8$ & $24 \cdot 25$ & 13 & 16 & $3+$ & $3+$ & 118 & $\sigma^{\pi} \mathrm{v}$ & $\dagger 0+33$ & 56 \\
\hline 567 & 1.5 & $4 \cdot 88$ & $11 \cdot 75$ & $12 \cdot 8$ & $14 \cdot 6$ & $18 \cdot 0$ & 22.95 & $24 \cdot 2$ & $26 \cdot 65$ & 15 & 17 & $3+17$ & $3+$ & 153 & o vi-iv & $1+32$ & 56 \\
\hline 568 & $1 \cdot 65$ & $5 \cdot 0$ & $12 \cdot 25$ & $13 \cdot 2$ & $15 \cdot 25$ & $18 \cdot 2$ & $23 \cdot 45$ & $24 \cdot 7$ & $27 \cdot 6$ & 15 & 16 & $3+16$ & $3+15$ & 175 & $\mathrm{o}^{\mathbf{v}}$ & $2+31$ & 55 \\
\hline 569 & 1.5 & $4 \cdot 7$ & $11 \cdot 1$ & 11.95 & $13 \cdot 8$ & $16 \cdot 5$ & $21 \cdot 55$ & $22 \cdot 7$ & $25 \cdot 5$ & 15 & 17 & $3+16$ & $3+14$ & 137 & ô. & $2+31$ & 55 \\
\hline 570 & 1.45 & $4 \cdot 6$ & $11 \cdot 36$ & $12 \cdot 6$ & $13 \cdot 95$ & $17 \cdot 25$ & $22 \cdot 3$ & $23 \cdot 5$ & $26 \cdot 2$ & 14 & 17 & $3+15$ & $3+14$ & 139 & ôv-v & $+2+31$ & 56 \\
\hline 571 & $1 \cdot 5$ & $4 \cdot 63$ & $11 \cdot 4$ & $12 \cdot 5$ & $14 \cdot 25$ & $17 \cdot 55$ & $22 \cdot 75$ & $24 \cdot 05$ & $26 \cdot 45$ & 15 & 16 & $3+17$ & $1+3+14$ & 146 & $\sigma^{2} \mathbf{v}-\mathbf{v i}$ & $1+33$ & 5 \\
\hline 572 & $1 \cdot 5$ & $4 \cdot 7$ & $11 \cdot 4$ & $12 \cdot 6$ & $14 \cdot 2$ & $17 \cdot 1$ & $22 \cdot 1$ & $23 \cdot 25$ & $26 \cdot 05$ & 15 & 17 & $3+16$ & $3+15$ & 150 & ô vi & $1+32$ & \\
\hline 573 & $1 \cdot 48$ & $4 \cdot 6$ & $11 \cdot 1$ & $12 \cdot 0$ & $13 \cdot 65$ & $16 \cdot 75$ & $21 \cdot 6$ & $22 \cdot 8$ & $25 \cdot 4$ & 15 & 17 & $3+15$ & $1+3+14$ & 113 & ơ vi-iii & $1+32^{2}$ & $=$ \\
\hline 574 & 1.5 & $4 \cdot 7$ & $12 \cdot 2$ & $12 \cdot 8$ & $14 \cdot 9$ & $18 \cdot 0$ & $23 \cdot 55$ & $24 \cdot 8$ & 27 . & 14 & 17 & $3+15$ & $3+13$ & 167 & $\sigma^{2} \mathrm{v}-\mathrm{vi}$ & $0+33$ & \\
\hline 575 & $1 \cdot 65$ & $5 \cdot 1$ & $12 \cdot 5$ & $13 \cdot 45$ & $15 \cdot:$ & $18 \cdot 6$ & 23 . & 24 & & 14 & 17 & $3+16$ & $4+13$ & 141 & ô vii & $1+31$ & \\
\hline 576 & $1 \cdot 54$ & $4 \cdot 85$ & $11 \cdot 9$ & $12 \cdot 4$ & $14 \cdot 55$ & $17 \cdot 2$ & $22 \cdot 35$ & $23 \cdot 6$ & $26 \cdot 3$ & 14 & 17 & $3+15$ & $3+15$ & 129 & 오 vii & $+0+32$ & \\
\hline
\end{tabular}




\begin{tabular}{|c|c|c|c|c|c|c|c|c|c|c|c|c|c|c|c|c|c|}
\hline 577 & $1 \cdot 46$ & $4 \cdot 7$ & $11 \cdot 8$ & $12 \cdot 6$ & $14 \cdot 3$ & $17 \cdot 65$ & $22 \cdot 7$ & $24 \cdot 1$ & $26 \cdot 8$ & 15 & 17 & $3+14$ & $3+14$ & 152 & 위 $\mathbf{v}$-vi & $2+32$ & 56 \\
\hline 578 & $1 \cdot 53$ & $4 \cdot 75$ & $11 \cdot 55$ & $12 \cdot 3$ & $14 \cdot 1$ & $17 \cdot 25$ & $22 \cdot 1$ & $23 \cdot 35$ & $25 \cdot 95$ & 13 & 17 & $3+16$ & $4+13$ & 127 & 오 vi-iii & $3+31$ & 55 \\
\hline 579 & $1 \cdot 7$ & $5 \cdot 1$ & $12 \cdot 35$ & $13 \cdot 4$ & $15 \cdot 6$ & $18 \cdot 55$ & $24 \cdot 0$ & $25 \cdot 4$ & $28 \cdot 15$ & 14 & 18 & $3+16$ & $3+13$ & 192 & 申 vi & $2+31$ & 55 \\
\hline 580 & 1.5 & $4 \cdot 75$ & $12 \cdot 45$ & $13 \cdot 25$ & $15 \cdot 4$ & $18 \cdot 8$ & $23 \cdot 9$ & $25 \cdot 2$ & $27 \cdot 8$ & 17 & 17 & $3+16$ & $3+14$ & 190 & 우 vi & $2+32^{*}$ & 56 \\
\hline 581 & $1 \cdot 5$ & $4 \cdot 6$ & $11 \cdot 35$ & $12 \cdot 05$ & $14 \cdot 3$ & 16.95 & $22 \cdot 3$ & $23 \cdot 5$ & $26 \cdot 2$ & 15 & 16 & $3+17$ & $4+15$ & 128 & 우 $\mathrm{iii}$ & $+0+33$ & 56 \\
\hline 582 & $1 \cdot 33$ & $4 \cdot 38$ & $10 \cdot 95$ & $11 \cdot 55$ & $13 \cdot 85$ & $16 \cdot 8$ & $21 \cdot 5$ & $22 \cdot 8$ & $25 \cdot 05$ & 15 & 17 & $3+17$ & $4+13$ & 131 & $\hat{o} \mathbf{v}-v i$ & $1+32^{*}$ & 55 \\
\hline 583 & $1 \cdot 5$ & $4 \cdot 65$ & $11 \cdot 55$ & $12 \cdot 6$ & $14 \cdot 4$ & $17 \cdot 45$ & $22 \cdot 85$ & $24 \cdot 0$ & $26 \cdot 7$ & 14 & 17 & $3+16$ & $3+16$ & 137 & ô vi-iii & $1+32$ & 55 \\
\hline 84 & $1 \cdot 74$ & $5 \cdot 2$ & $12 \cdot 5$ & $13 \cdot 3$ & $15 \cdot 45$ & $18 \cdot 7$ & $23 \cdot 95$ & $25 \cdot 4$ & $28 \cdot 05$ & 15 & 18 & $3+16$ & $3+14$ & 169 & ठే v-vi & $2+31$ & 55 \\
\hline 585 & 1.45 & $4 \cdot 6$ & $11 \cdot 35$ & $12 \cdot 2$ & $14 \cdot 15$ & $17 \cdot 35$ & $22 \cdot 3$ & $23 \cdot 45$ & $26 \cdot 05$ & 16 & 16 & $2+16$ & $3+13$ & 151 & oै v-vi & $2+32$ & 56 \\
\hline 586 & $1 \cdot 56$ & $4 \cdot 75$ & $11 \cdot 75$ & $12 \cdot 9$ & $14 \cdot 5$ & $18 \cdot 45$ & $23 \cdot 4$ & $24 \cdot 65$ & $27 \cdot 3$ & 15 & 17 & $2+16$ & $3+14$ & 167 & 우 vi & $1+32$ & 56 \\
\hline 87 & $1 \cdot 44$ & $4 \cdot 6$ & $11 \cdot 3$ & $12 \cdot 25$ & $14 \cdot 05$ & $17 \cdot 5$ & 22.45 & $23 \cdot 65$ & $26 \cdot 25$ & 15 & $\begin{array}{l}\text { Rt. broken, } \\
\text { left } 17 \text {. }\end{array}$ & $3+16$ & $3+14$ & 144 & ơ iv-v & $2+32$ & 56 \\
\hline 588 & $1 \cdot 7$ & $5 \cdot 05$ & $12 \cdot 3$ & $12 \cdot 9$ & $15 \cdot 2$ & $18 \cdot 3$ & $23 \cdot 4$ & $24 \cdot 7$ & $27 \cdot 3$ & 15 & 18 & $3+16$ & $3+14$ & 145 & ô vi-iii & $1+33$ & $56^{*}$ \\
\hline 39 & $1 \cdot 78$ & $5 \cdot 26$ & $12 \cdot 6$ & $13 \cdot 1$ & $15 \cdot 6$ & $18 \cdot 0$ & $24 \cdot 3$ & $25 \cdot 65$ & 28.45 & 14 & 17 & $3+16$ & $4+14$ & 157 & 오 vii & $1+32$ & 57 \\
\hline 90 & $1 \cdot 48$ & $4 \cdot 65$ & $11 \cdot 85$ & $12 \cdot 45$ & $14 \cdot 7$ & $17 \cdot 45$ & $22 \cdot 4$ & $23 \cdot 65$ & $26 \cdot 3$ & 14 & 17. & $3+17$ & $4+14$ & 140 & $\delta$ v-vi & $\dagger 2+32$ & 56 \\
\hline 1 & $1 \cdot 6$ & $5 \cdot 0$ & $11 \cdot 75$ & $12 \cdot 7$ & $14 \cdot 85$ & $17 \cdot 75$ & $22 \cdot 8$ & $24 \cdot 05$ & $26 \cdot 85$ & 14 & 17 & $3+16$ & $3+13$ & 166 & o vi-iv & $3+31$ & 55 \\
\hline 92 & 1.5 & $4 \cdot 6$ & $11 \cdot 5$ & $12 \cdot 3$ & $14 \cdot 4$ & 16.95 & $22 \cdot 15$ & $23 \cdot 4$ & $26 \cdot 15$ & 14 & 16 & $2+16$ & $3+15$ & 119 & 우 vi-iv & $2+32$ & 56 \\
\hline 3 & $1 \cdot 43$ & $4 \cdot 43$ & $10 \cdot 95$ & $11 \cdot 6$ & $13 \cdot 85$ & $16 \cdot 3$ & $21 \cdot 75$ & $22 \cdot 95$ & $25 \cdot 4$ & 15 & 17 & $3+17$ & $4+15$ & 128 & $\hat{o} \mathrm{v}-\mathrm{vi}$ & $0+33$ & 56 \\
\hline 94 & $1 \cdot 5$ & $4 \cdot 85$ & $11 \cdot 8$ & $12 \cdot 65$ & $14 \cdot 8$ & $17 \cdot 35$ & $22 \cdot 65$ & $23 \cdot 9$ & $26 \cdot 5$ & 15 & 17 & $2+17$ & $3+14$ & 158 & $\hat{o} \mathrm{v}-\mathrm{vi}$ & $1+32$ & 56 \\
\hline 95 & $1 \cdot 4$ & $4 \cdot 47$ & $11 \cdot 15$ & 11.55 & $13 \cdot 9$ & $16 \cdot 65$ & $21 \cdot 75$ & $23 \cdot 0$ & $25 \cdot 3$ & 16 & 16 & $4+16$ & $3+14$ & 136 & ô vi & $1+33$ & 56 \\
\hline 96 & $1 \cdot 54$ & $4 \cdot 7$ & $12 \cdot 1$ & $12 \cdot 5$ & $15 \cdot 05$ & $17 \cdot 9$ & $23 \cdot 2$ & $24 \cdot 4$ & 26.95 & 16 & 16 & $3+17$ & $3+14$ & 170 & o vi & $1+33$ & 56 \\
\hline 77 & 1.78 & $5 \cdot 35$ & $12 \cdot 25$ & $12 \cdot 9$ & $15 \cdot 3$ & $18 \cdot 2$ & 23.95 & $25 \cdot 2$ & $28 \cdot 1$ & 15 & 17 & $3+16$ & $3+13$ & 171 & $0^{0} \mathbf{v}$ & $1+33$ & 56 \\
\hline 98 & $1 \cdot 48$ & $4 \cdot 55$ & $11 \cdot 2$ & $12 \cdot 1$ & $14 \cdot 0$ & $16 \cdot 85$ & $21 \cdot 95$ & $23 \cdot 1$ & $25 \cdot 65$ & 15 & 17 & $3+16$ & $3+15$ & 134 & ôt-vi & $+0+34$ & 57 \\
\hline 99 & $1 \cdot 56$ & $4 \cdot 9$ & $12 \cdot 25$ & 13.05 & $15 \cdot 5$ & $18 \cdot 3$ & $23 \cdot 7$ & $24 \cdot 9$ & $28 \cdot 0$ & 15 & 17 & $3+17$ & $3+15$ & 151 & of vii & $2+31$ & 56 \\
\hline 0 & $1 \cdot 5$ & $4 \cdot 4$ & $10 \cdot 85$ & $11 \cdot 6$ & $13 \cdot 7$ & $16 \cdot 2$ & $21 \cdot 2$ & $22 \cdot 3$ & $24 \cdot 85$ & 15 & 17 & $3+16$ & $4+13$ & 123 & ô vi-iii & $1+31$ & 55 \\
\hline 1 & $1 \cdot 68$ & $5 \cdot 28$ & $13 \cdot 3$ & $14 \cdot 2$ & $16 \cdot 5$ & $19 \cdot 65$ & $25 \cdot 4$ & $26 \cdot 75$ & $29 \cdot 6$ & 14 & 17 & $3+16$ & $3+14$ & 207 & vi & $1+32$ & 56 \\
\hline 2 & $1 \cdot 65$ & $4 \cdot 9$ & $12 \cdot 06$ & $13 \cdot 1$ & $15 \cdot 1$ & $18 \cdot 1$ & $23 \cdot 2$ & $24 \cdot 4$ & $27 \cdot 1$ & 15 & 17 & $2+16$ & $2+14$ & 180 & ơ vi & $2+32$ & 56 \\
\hline 3 & $1 \cdot 6$ & $5 \cdot 0$ & $12 \cdot 1$ & $12 \cdot 6$ & $15 \cdot 1$ & $17 \cdot 6$ & $23 \cdot 3$ & $24 \cdot 55$ & $27 \cdot 1$ & 14 & 17 & $3+16$ & $4+15$ & 157 & 우 iii & $2+32$ & 56 \\
\hline 4 & $1 \cdot 6$ & $5 \cdot 0$ & $11 \cdot 8$ & 12.95 & $14 \cdot 8$ & $17 \cdot 95$ & $23 \cdot 15$ & $24 \cdot 4$ & $27 \cdot 35$ & 14 & 17 & $3+16$ & $3+16$ & 149 & ㅇ. vi-iv & $\dagger 1+33$ & 56 \\
\hline 5 & $1 \cdot 38$ & $4 \cdot 4$ & $11 \cdot 1$ & $11 \cdot 85$ & $13 \cdot 8$ & $16 \cdot 6$ & $21 \cdot 75$ & $22 \cdot 95$ & $25 \cdot 55$ & 15 & 16 & $3+16$ & $3+15$ & 137 & ô $\mathrm{v}-\mathrm{vi}$ & $\dagger 0+32$ & 55 \\
\hline 96 & $1 \cdot 56$ & $4 \cdot 7$ & $11 \cdot 3$ & $12 \cdot 4$ & $14 \cdot 2$ & $17 \cdot 65$ & $22 \cdot 7$ & $24 \cdot 0$ & $26 \cdot 7$ & 16 & 16 & $3+16$ & $3+15$ & 160 & ơ vi & $1+32$ & 56 \\
\hline 77 & $1 \cdot 52$ & $4 \cdot 65$ & $11 \cdot 5$ & 12.5 & $14 \cdot 55$ & $17 \cdot 6$ & $22 \cdot 65$ & $23 \cdot 9$ & $26 \cdot 4$ & 16 & 16 & $3+16$ & $4+13$ & 157 & q vi & $1+32$ & 55 \\
\hline be & $1 \cdot 68$ & $5 \cdot 14$ & $12 \cdot 3$ & $13 \cdot 00$ & $15 \cdot 55$ & 18.55 & $24 \cdot 0$ & $25 \cdot 3$ & $28 \cdot 2$ & 15 & 17 & $3+16$ & $3+14$ & 176 & 우 vi-iii & $2+31$ & 55 \\
\hline 609 & $1 \cdot 6$ & $4 \cdot 9$ & $11 \cdot 8$ & 12.4 & $14 \cdot 65$ & $17 \cdot 55$ & $23 \cdot 0$ & $24 \cdot 2$ & $26 \cdot 7$ & 15 & 17 & $3+16$ & $3+15$ & 128 & ô vi-iii & $+0+34$ & 57 \\
\hline 610 & $1 \cdot 4$ & $4 \cdot 6$ & $11 \cdot 6$ & $12 \cdot 3$ & $14 \cdot 3$ & $17 \cdot 3$ & $22 \cdot 3$ & $23 \cdot 45$ & $26 \cdot 1$ & 14 & 16 & $3+16$ & $3+15$ & 137 & oi-iv & $+0+33$ & 56 \\
\hline 11 & $1 \cdot 44$ & $4 \cdot 6$ & $11 \cdot 15$ & $11 \cdot 7$ & 13.85 & 16.45 & $21 \cdot 65$ & $22 \cdot 8$ & $25 \cdot 3$ & 15 & 17 & $3+17$ & $3+14$ & 106 & $\mathrm{o}^{x} \mathrm{iv}-\mathrm{vi}$ & $2+32$ & 56 \\
\hline 2. & $1 \cdot 48$ & $4 \cdot 45$ & $10 \cdot 65$ & $10 \cdot 95$ & $13 \cdot 35$ & $15 \cdot 65$ & $20 \cdot 55$ & $21 \cdot 65$ & $24 \cdot 15$ & 15 & 16 & $3+17$ & $3+14$ & 117 & ô vi & $0+33$ & 56 \\
\hline 89 & $1 \cdot 3$ & $4 \cdot 15$ & $10 \cdot 2$ & $10 \cdot 55$ & $12 \cdot 7$ & $15 \cdot 1$ & $19 \cdot 55$ & $20 \cdot 6$ & $22 \cdot 7$ & 15 & 18 & $3+16$ & $3+14$ & 101 & $\hat{\sigma} \mathbf{v}-v i$ & $2+32$ & 56 \\
\hline 6 & $1 \cdot 25$ & $4 \cdot 2$ & $10 \cdot 15$ & $10 \cdot 7$ & $12 \cdot 85$ & $15 \cdot 05$ & $19 \cdot 85$ & $21 \cdot 0$ & $23 \cdot 3$ & 16 & 17 & $2+17$ & $3+13$ & 108 & 오 vi-iii & $1+32$ & 56 \\
\hline 61 & $1 \cdot 4$ & $4 \cdot 45$ & $11 \cdot 25$ & $12 \cdot 2$ & $14 \cdot 1$ & $17 \cdot 05$ & $22 \cdot 35$ & $23 \cdot 6$ & $26 \cdot 3$ & 15 & 18 & $3+16$ & $4+13$ & 165 & ô vi & $1+32$ & 55 \\
\hline 010 & $1 \cdot 6$ & $5 \cdot 0$ & $12 \cdot 4$ & $13 \cdot 6$ & $15 \cdot 6$ & 18.95 & $24 \cdot 2$ & $25 \cdot 6$ & $28 \cdot 45$ & 14 & 17 & $3+16$ & $3+15$ & 200 & vi & $1+31$ & 55 \\
\hline 017 & $1 \cdot 73$ & $5 \cdot 3$ & $12 \cdot 05$ & $12 \cdot 80$ & $15 \cdot 35$ & $17 \cdot 5$ & $23 \cdot 0$ & $24 \cdot 3$ & $27 \cdot 1$ & 15 & 17 & $3+17$ & $3+14$ & 159 & 오 vi-iii & $1+32$ & 56 \\
\hline
\end{tabular}




\begin{tabular}{|c|c|c|c|c|c|c|c|c|c|c|c|c|c|c|c|c|c|}
\hline $\begin{array}{l}\text { No. of } \\
\text { fish. }\end{array}$ & 1 & 2 & 3 & 4 & 5 & 6 & 7 & 8 & 9 & 11 & 12 & 13 & 14 & 15 & 16 & 17 & 18 \\
\hline 618 & $1 \cdot 47$ & $4 \cdot 7$ & $11 \cdot 7$ & $12 \cdot 7$ & $14 \cdot 55$ & $17 \cdot 5$ & $22 \cdot 6$ & $23 \cdot 9$ & $26 \cdot 65$ & 15 & 17 & $3+16$ & $4+13$ & 154 & 오 vi-iv & $+0+33$ & 56 \\
\hline 619 & $1 \cdot 56$ & $4 \cdot 9$ & $12 \cdot 2$ & $12 \cdot 9$ & $15 \cdot 05$ & $17 \cdot 8$ & $23 \cdot 15$ & $24 \cdot 35$ & $27 \cdot 1$ & 14 & 17 & $3+16$ & $4+14$ & 188 & 우 vi & $0+32$ & 57 \\
\hline 620 & $1 \cdot 64$ & $4 \cdot 8$ & $12 \cdot 1$ & $12 \cdot 6$ & $14 \cdot 9$ & $17 \cdot 8$ & $22 \cdot 85$ & $24 \cdot 15$ & $26 \cdot 8$ & 15 & 17 & $3+16$ & $4+15$ & 146 & o vi-iii & $2+32$ & 56 \\
\hline 621 & $1 \cdot 63$ & $4 \cdot 95$ & $11 \cdot 45$ & $12 \cdot 45$ & $14 \cdot 5$ & $17 \cdot 3$ & $22 \cdot 6$ & $23 \cdot 85$ & $26 \cdot 45$ & 14 & 16 & $3+17$ & $4+13$ & 136 & q vi-iii & $0+33$ & 56 \\
\hline 622 & $1 \cdot 4$ & $4 \cdot 6$ & $11 \cdot 25$ & $12 \cdot 3$ & $14 \cdot 2$ & $17 \cdot 4$ & $22 \cdot 45$ & $23 \cdot 8$ & $26 \cdot 2$ & 15 & 17 & $3+17$ & $3+13$ & 160 & $\delta \mathrm{v}$-vi & $0+32$ & 56 \\
\hline 623 & 1.45 & $4 \cdot 55$ & $11 \cdot 4$ & $12 \cdot 4$ & $14 \cdot 2$ & $17 \cdot 0$ & $22 \cdot 4$ & $23 \cdot 6$ & $26 \cdot 05$ & 14 & 17 & $3+15$ & $2+14$ & 143 & ơ vi-iv & $0+34$ & 56 \\
\hline 624 & $1 \cdot 65$ & $4 \cdot 9$ & $11 \cdot 75$ & $12 \cdot 35$ & $14 \cdot 7$ & $17 \cdot 65$ & $22 \cdot 7$ & 23.95 & 26.8 & 14 & 17 & $3+16$ & $3+15$ & 165 & 우 vi & $0+33$ & 56 \\
\hline 625 & $1 \cdot 64$ & $4 \cdot 95$ & $12 \cdot 3$ & $13 \cdot 6$ & $15 \cdot 25$ & $18 \cdot 9$ & $24 \cdot 0$ & $25 \cdot 4$ & $28 \cdot 15$ & 15 & 17 & $3+15$ & $3+12$ & 199 & 우 vi & $1+33$ & 57 \\
\hline 626 & $1 \cdot 76$ & $5 \cdot 43$ & $13 \cdot 1$ & $14 \cdot 4$ & $16 \cdot 5$ & $19 \cdot 75$ & $25 \cdot 35$ & $26 \cdot 8$ & $29 \cdot 95$ & 15 & 18 & $3+16$ & $3+15$ & 187 & $\sigma^{t} \mathrm{vi}$ & $0+33$ & 56 \\
\hline 627 & $1 \cdot 6$ & $5 \cdot 1$ & $12 \cdot 6$ & $13 \cdot 3$ & $15 \cdot 8$ & $18 \cdot 45$ & $24 \cdot 25$ & $25 \cdot 55$ & $28 \cdot 65$ & 15 & 17 & $2+17$ & $3+16$ & 180 & $\mathrm{o}^{7} \mathrm{vi}$ & $0+33$ & 56 \\
\hline 628 & $1 \cdot 6$ & $5 \cdot 13$ & $12 \cdot 4$ & $13 \cdot 2$ & $15 \cdot 2$ & 18.65 & $23 \cdot 8$ & $25 \cdot 05$ & $27 \cdot 35$ * & 16 & 16 & $3+15$ & $3+13$ & 136 & ô ii & $1+31$ & 55 \\
\hline 629 & $1 \cdot 43$ & $4 \cdot 45$ & $10 \cdot 8$ & $11 \cdot 7$ & $13 \cdot 4$ & $16 \cdot 6$ & $21 \cdot 4$ & 22.55 & $25 \cdot 1$ & 15 & 19 & $3+16$ & $3+13$ & 142 & ovi & $1+32$ & 56 \\
\hline 630 & $1 \cdot 53$ & $4 \cdot 85$ & $12 \cdot 0$ & $12 \cdot 8$ & $14 \cdot 95$ & $17 \cdot 7$ & $23 \cdot 2$ & $24 \cdot 45$ & $27 \cdot 2$ & 14 & 17 & $3+16$ & $3+14$ & 148 & ô vi-iii & $1+31$ & 55 \\
\hline 631 & 1.52 & $5 \cdot 0$ & $12 \cdot 2$ & $12 \cdot 8$ & $14 \cdot 95$ & 18.05 & $22 \cdot 3$ & $24 \cdot 6$ & $27 \cdot 5$ & 14 & 17 & $3+16$ & $3+14$ & 175 & ô vi $\quad 0$ & $+[1+1+30]$ & 55 \\
\hline 632 & $1 \cdot 6$ & $5 \cdot 0$ & $12 \cdot 55$ & $13 \cdot 00$ & $15 \cdot 55$ & $18 \cdot 35$ & $24 \cdot 15$ & $25 \cdot 4$ & $27 \cdot 95$ & 15 & 18 & $3+16$ & $3+15$ & 168 & 우 vi & $0+33$ & 57 \\
\hline 633 & $1 \cdot 6$ & $4 \cdot 85$ & $12 \cdot 45$ & $13 \cdot 3$ & $15 \cdot 45$ & $18 \cdot 6$ & $24 \cdot 1$ & $25 \cdot 45$ & $27 \cdot 7 *$ & 14 & 18 & $3+15$ & $3+14$ & 171 & ô vi & $1+32$ & 56 \\
\hline 634 & 1.5 & $4 \cdot 42$ & $11 \cdot 5$ & $12 \cdot 4$ & $14 \cdot 05$ & $17 \cdot 4$ & $22 \cdot 4$ & $23 \cdot 65$ & $26 \cdot 15$ & 15 & 17 & $3+16$ & $3+14$ & 148 & o iii-iv & $1+33$ & 56 \\
\hline 635 & $1 \cdot 4$ & $4 \cdot 7$ & $11 \cdot 3$ & $11 \cdot 7$ & $14 \cdot 05$ & $16 \cdot 75$ & $21 \cdot 8$ & $23 \cdot 05$ & $25 \cdot 65$ & 14 & 17 & $3+17$ & $3+13$ & 117 & ơ vi-iii & $1+32$ & 56 \\
\hline 636 & $1 \cdot 53$ & $4 \cdot 9$ & $11 \cdot 8$ & $12 \cdot 45$ & $14 \cdot 9$ & $17 \cdot 6$ & $22 \cdot 8$ & $24 \cdot 05$ & $26 \cdot 75$ & 14 & 17 & $3+17$ & $2+14$ & 140 & ô vi & $0+32$ & 55 \\
\hline 637 & 1.5 & $4 \cdot 65$ & $11 \cdot 4$ & $12 \cdot 6$ & $14 \cdot 35$ & $17 \cdot 5$ & $22 \cdot 4$ & $23 \cdot 65$ & $26 \cdot 4$ & 15 & 17 & $3+16$ & $2+14$ & 145 & yi & $2+31$ & 56 \\
\hline 638 & $1 \cdot 4$ & $4 \cdot 7$ & $12 \cdot 3$ & $12 \cdot 6$ & $15 \cdot 15$ & $17 \cdot 75$ & $22 \cdot 75$ & 23.95 & $26 \cdot 5$ & 15 & 17 & $3+16$ & $3+14$ & 156 & क vi & $+0+32$ & 56 \\
\hline 639 & 1.55 & $4 \cdot 85$ & $11 \cdot 6$ & $12 \cdot 85$ & $14 \cdot 6$ & $17 \cdot 8$ & $22 \cdot 8$ & $24 \cdot 1$ & $26 \cdot 75$ & 15 & 16 & $3+17$ & $3+14$ & 141 & 우 vi-iv & $1+32$ & 55 \\
\hline 640 & $1 \cdot 43$ & $4 \cdot 6$ & $11 \cdot 8$ & $12 \cdot 5$ & $14 \cdot 65$ & $17 \cdot 75$ & $22 \cdot 9$ & $24 \cdot 15$ & $26 \cdot 8$ & 15 & 16 & $3+17$ & $2+14$ & 144 & o vi & $2+32$ & 57 \\
\hline 641 & $1 \cdot 54$ & $4 \cdot 9$ & $11 \cdot 8$ & $12 \cdot 7$ & $14 \cdot 65$ & $17 \cdot 5$ & $22 \cdot 3$ & $23 \cdot 6$ & $26 \cdot 25$ & 15 & 17 & $3+15$ & $3+14$ & 138 & 우 vi-iii & $1+31$ & 55 \\
\hline 642 & $1 \cdot 43$ & $4 \cdot 65$ & $11 \cdot 5$ & $12 \cdot 25$ & $14 \cdot 2$ & $17 \cdot 1$ & $22 \cdot 0$ & $23 \cdot 1$ & $25 \cdot 95$ & 14 & $16^{*}$ & $3+16$ & $3+14$ & 137 & ô vi & $1+32$ & 56 \\
\hline 643 & $1 \cdot 47$ & $4 \cdot 44$ & $10 \cdot 9$ & $11 \cdot 55$ & $13 \cdot 55$ & $16 \cdot 0$ & $21 \cdot 15$ & $22 \cdot 35$ & $24 \cdot 85$ & 14 & 16 & $3+16$ & $4+14$ & 129 & ô vi & $1+33$ & 56 \\
\hline 644 & $1 \cdot 6$ & $4 \cdot 9$ & $12 \cdot 1$ & $12 \cdot 7$ & $14 \cdot 75$ & $17 \cdot 9$ & $23 \cdot 15$ & $24: 35$ & $27 \cdot 15$ & 15 & 17 & $3+16$ & $3+15$ & 148 & $\hat{o}^{2} \mathrm{v}-\mathrm{vi}$ & $0+32$ & 55 \\
\hline 645 & $1 \cdot 48$ & $4 \cdot 6$ & $11 \cdot 15$ & $11 \cdot 85$ & $13 \cdot 9$ & $16 \cdot 7$ & $21 \cdot 8$ & $22 \cdot 9$ & $25 \cdot 5$ & 16 & 16 & $4+16$ & $3+14$ & 127 & o iii & $1+33$ & 57 \\
\hline 646 & 1.55 & $4 \cdot 8$ & $12 \cdot 0$ & $12 \cdot 7$ & $14 \cdot 8$ & $17 \cdot 9$ & $23 \cdot 15$ & $24 \cdot 5$ & $27 \cdot 2$ & 14 & 18 & $3+16$ & $3+14$ & 177 & ㅇv vi & $1+30$ & 54 \\
\hline 647 & $1 \cdot 48$ & $4 \cdot 65$ & 11.55 & $12 \cdot 5$ & $14: 35$ & $17 \cdot 5$ & $22 \cdot 25$ & $23 \cdot 6$ & $26 \cdot 35$ & 14 & 16 & $3+16$ & $2+13$ & 152 & ㅇ vi & $0+32$ & 55 \\
\hline 648 & $1 \cdot 67$ & $5 \cdot 37$ & $12 \cdot 95$ & $13 \cdot 7$ & $16 \cdot 05$ & $19 \cdot 2$ & $24 \cdot 65$ & $25 \cdot 95$ & $28 \cdot 7$ & 17 & 17 & $3+16$ & $1+3+14$ & 181 & 우 $\mathrm{y}$ & $2+32$ & 56 \\
\hline 649 & $1 \cdot 6$ & $4 \cdot 85$ & $11 \cdot 9$ & $12 \cdot 45$ & $14 \cdot 75$ & $17 \cdot 6$ & $22 \cdot 75$ & $24 \cdot 0$ & $26 \cdot 7$ & 14 & 17 & $3+17$ & $3+15$ & 152 & $\sigma \mathrm{ii}$ & $1+32$ & 55 \\
\hline 650 & $1 \cdot 5$ & $5 \cdot 0$ & $12 \cdot 45$ & $13 \cdot 0$ & $15 \cdot 4$ & $18 \cdot 3$ & $23 \cdot 9$ & $25 \cdot 15$ & $27 \cdot 8$ & 14 & 16 & $3+17$ & $3+15$ & 170 & ovi & $1+33$ & 57 \\
\hline 651 & $1 \cdot 6$ & $4 \cdot 8$ & $11 \cdot 4$ & $12 \cdot 2$ & $14 \cdot 35$ & $17 \cdot 35$ & $22 \cdot 5$ & $23 \cdot 7$ & $26 \cdot 4$ & 15 & 17 & $3+16$ & $3+13$ & 161 & 우 vi & $1+32$ & 55 \\
\hline 652 & $1 \cdot 4$ & $4 \cdot 6$ & $11 \cdot 7$ & $12 \cdot 7$ & $14 \cdot 4$ & $17 \cdot 7$ & $22 \cdot 9$ & $24 \cdot 2$ & $26 \cdot 8$ & 15 & 16 & $3+15$ & $3+14$ & 149 & ơ vi-iii & $2+32$ & 55 \\
\hline 653 & $1 \cdot 63$ & $5 \cdot 05$ & $11 \cdot 75$ & $12 \cdot 4$ & $14 \cdot 7$ & $17 \cdot 4$ & $22 \cdot 55$ & $23 \cdot 8$ & $26 \cdot 6$ & 15 & 17 & $3+17$ & $4+14$ & 135 & $\hat{0} \mathbf{y}$ & $1+32$ & 55 \\
\hline 654 & $1 \cdot 38$ & $4 \cdot 35$ & $10 \cdot 45$ & $11 \cdot 2$ & $13 \cdot 1$ & $15 \cdot 95$ & $20 \cdot 8$ & $22 \cdot 0$ & $24 \cdot 5$ & 15 & 18 & $3+17$ & $4+13$ & 114 & of vi-iii & $\dagger 2+32$ & 57 \\
\hline 655 & $1 \cdot 6$ & $4 \cdot 7$ & $11 \cdot 6$ & $12 \cdot 3$ & $14 \cdot 3$ & $17 \cdot 35$ & $22 \cdot 25$ & $23 \cdot 45$ & $26 \cdot 19+0$ & $0+0+0+3$ & 317 & $3+17$ & $3+14$ & 140 & ô vi & $2+32$ & 56 \\
\hline 656 & 1.42 & 4.55 & $11 \cdot 45$ & $12 \cdot 25$ & $14 \cdot 2$ & $17 \cdot 2$ & $22 \cdot 55$ & $23 \cdot 8$ & $26 \cdot 2$ & 15 & 17 & $3+16$ & $3+15$ & 132 & $\sigma^{\lambda} \mathrm{v}-\mathrm{vi}$ & $1+33$ & 56 \\
\hline
\end{tabular}




\begin{tabular}{|c|c|c|c|c|c|c|c|c|c|c|c|c|c|c|c|c|}
\hline 657 & 1.5 & $4 \cdot 7$ & $12 \cdot 0$ & $12 \cdot 55$ & $14 \cdot 75$ & $17 \cdot 5$ & $22 \cdot 8$ & $24 \cdot 05$ & $26 \cdot 7$ & 14 & 17 & $3+16$ & $3+15$ & 152 & 우 vi & $1+32$ \\
\hline 658 & $1 \cdot 58$ & $5 \cdot 0$ & $12 \cdot 4$ & $12 \cdot 95$ & $15 \cdot 35$ & $18 \cdot 0$ & $23 \cdot 25$ & $24 \cdot 45$ & $27 \cdot 1$ & 14 & 17 & $3+16$ & $3+14$ & 150 & $\hat{o v}$ & $2+31$ \\
\hline 659 & $1 \cdot 6$ & $4 \cdot 7$ & $11 \cdot 2$ & $12 \cdot 2$ & $14 \cdot 05$ & $16 \cdot 5$ & $21 \cdot 55$ & $22 \cdot 8$ & $25 \cdot 3$ & 14 & 17 & $3+17$ & $3+15$ & 136 & 우 vi & $0+33$ \\
\hline 660 & $1 \cdot 5$ & $5 \cdot 0$ & $12 \cdot 3$ & $12 \cdot 8$ & $15 \cdot 2$ & $18 \cdot 0$ & $23 \cdot 4$ & $24 \cdot 5$ & $27 \cdot 4$ & 14 & 17 & $3+16$ & $1+4+13$ & 155 & $\widehat{\sigma} v-v i$ & $2+32$ \\
\hline 661 & $1 \cdot 47$ & $4 \cdot 7$ & $11 \cdot 65$ & $12 \cdot 3$ & $14 \cdot 6$ & $17 \cdot 3$ & $22 \cdot 55$ & $23 \cdot 8$ & $26 \cdot 6$ & 14 & 16 & $3+16$ & $3+14$ & 160 & ô vi & $2+31$ \\
\hline 662 & $1 \cdot 45$ & $4 \cdot 6$ & $11 \cdot 05$ & $11 \cdot 85$ & $13 \cdot 65$ & $16 \cdot 25$ & $21 \cdot 2$ & $22 \cdot 45$ & $25 \cdot 15$ & 14 & 16 & $3+16$ & $3+14$ & 128 & $\hat{\sigma} v-v i$ & $2+30$ \\
\hline 663 & $1 \cdot 55$ & $5 \cdot 15$ & $12 \cdot 75$ & $13 \cdot 25$ & $15 \cdot 8$ & $18 \cdot 8$ & $24 \cdot 15$ & $25 \cdot 5$ & $28 \cdot 35$ & 16 & 17 & $3+16$ & $3+14$ & 144 & $\sigma^{*} \mathrm{ii}$ & $2+31$ \\
\hline 664 & $1 \cdot 36$ & $4 \cdot 42$ & $11 \cdot 1$ & $11 \cdot 7$ & $13 \cdot 85$ & $16 \cdot 55$ & $21 \cdot 6$ & $22 \cdot 7$ & $25 \cdot 2$ & 16 & 18 & $3+16$ & $3+16$ & 135 & $\sigma^{*} v-v i$ & $0+32$ \\
\hline 665 & $1 \cdot 34$ & $4 \cdot 36$ & $10 \cdot 75$ & $11 \cdot 5$ & $13 \cdot 2$ & $16 \cdot 35$ & $21 \cdot 3$ & $22 \cdot 4$ & $24 \cdot 9$ & 15 & 17 & $3+15$ & $3+14$ & 125 & of v-vi & $0+33$ \\
\hline 666 & $1 \cdot 5$ & $4 \cdot 9$ & $12 \cdot 3$ & $12 \cdot 85$ & $15 \cdot 15$ & $18 \cdot 4$ & $23 \cdot 5$ & $24 \cdot 75$ & $27 \cdot 6$ & 16 & 18 & $3+15$ & $3+15$ & 164 & $\sigma v-v i$ & $1+33$ \\
\hline 667 & $1 \cdot 5$ & $4 \cdot 6$ & $11 \cdot 0$ & $11 \cdot 8$ & $13 \cdot 65$ & $16 \cdot 6$ & $21 \cdot 5$ & $22 \cdot 55$ & $25 \cdot 2$ & 15 & 17 & $3+16$ & $3+13$ & 118 & ô ii & $1+32$ \\
\hline 668 & $1 \cdot 38$ & $4 \cdot 3$ & $10 \cdot 75$ & $11 \cdot 3$ & $13 \cdot 4$ & $16 \cdot 0$ & $20 \cdot 65$ & $21 \cdot 8$ & $24 \cdot 4$ & 15 & 16 & $3+16$ & $3+14$ & 119 & ơ iii & $1+31$ \\
\hline 669 & $1 \cdot 4$ & $4 \cdot 5$ & $11 \cdot 1$ & $11 \cdot 75$ & $13 \cdot 95$ & $16 \cdot 6$ & $22 \cdot 1$ & $23 \cdot 3$ & $25 \cdot 8 \dagger$ & 14 & 18 & $3+16$ & $3+15$ & 139 & q vi & $1+31$ \\
\hline 670 & $1 \cdot 46$ & $4 \cdot 45$ & $10 \cdot 9$ & $11 \cdot 7$ & $13 \cdot 7$ & $16 \cdot 75$ & $21 \cdot 7$ & $22 \cdot 9$ & $25 \cdot 4$ & 15 & 17 & $3+17$ & $3+16$ & 119 & ô vi & $0+32$ \\
\hline 671 & $1 \cdot 35$ & $4 \cdot 5$ & $10 \cdot 7$ & $11 \cdot 9$ & $13 \cdot 65$ & $16 \cdot 6$ & $21 \cdot 75$ & $23 \cdot 0$ & $25 \cdot 55$ & 15 & 17 & $3+16$ & $3+14$ & 128 & o i-ii & $1+32$ \\
\hline 672 & $1 \cdot 6$ & $5 \cdot 05$ & $12 \cdot 1$ & $13 \cdot 0$ & $15 \cdot 1$ & $18 \cdot 2$ & $23 \cdot 9$ & $25 \cdot 2$ & $27 \cdot 81$ & $-0+11$ & 17 & $3+17$ & $3+14$ & 169 & iv & $1+33$ \\
\hline 673 & $1 \cdot 52$ & $4 \cdot 55$ & $11 \cdot 55$ & $12 \cdot 1$ & $14 \cdot 45$ & $17 \cdot 35$ & $22 \cdot 5$ & $23 \cdot 75$ & $26 \cdot 45$ & 15 & 16 & $3+16$ & $1+3+13$ & 128 & vi-iii & $1+32$ \\
\hline 674 & $1 \cdot 5$ & $4 \cdot 53$ & $11 \cdot 55$ & $12 \cdot 8$ & $14 \cdot 45$ & $17 \cdot 9$ & $22 \cdot 9$ & $24 \cdot 1$ & $26 \cdot 8$ & 14 & 16 & $3+16$ & $\begin{array}{l}\text { Total 17. } \\
\text { Rays broken. }\end{array}$ & 140 & vi-iii & $0+33$ \\
\hline 675 & $1 \cdot 38$ & $4 \cdot 35$ & $11 \cdot 0$ & $11 \cdot 65$ & $13 \cdot 4$ & $16 \cdot 3$ & $21 \cdot 15$ & $22 \cdot 25$ & $24 \cdot 85$ & 14 & 17 & $3+15$ & $3+15$ & 110 & vi-iii & $0+33$ \\
\hline 676 & $1 \cdot 4$ & $4 \cdot 68$ & $11 \cdot 4$ & $12 \cdot 35$ & $14 \cdot 3$ & $17 \cdot 3$ & $22 \cdot 5$ & $23 \cdot 7$ & $26 \cdot 5$ & 15 & 17 & $3+16$ & $3+14$ & 137 & q iv-v & $\dagger 0+33$ \\
\hline 677 & $1 \cdot 4$ & $4 \cdot 7$ & $11 \cdot 5$ & $12 \cdot 35$ & $14 \cdot 45$ & $17 \cdot 0$ & $22 \cdot 35$ & $23 \cdot 7$ & $26 \cdot 3$ & 15 & 17 & $3+16$ & $1+3+14$ & 144 & ovi & $1+31$ \\
\hline 678 & $1 \cdot 4$ & $4 \cdot 52$ & $10 \cdot 9$ & $11 \cdot 9$ & $13 \cdot 65$ & $16 \cdot 6$ & $21 \cdot 75$ & $22 \cdot 95$ & $25 \cdot 4$ & 15 & 17 & $3+16$ & $3+14$ & 108 & vii & $2+31$ \\
\hline 679 & $1 \cdot 45$ & $4 \cdot 65$ & 11.5 & $12 \cdot 5$ & $14 \cdot 3$ & $17 \cdot 3$ & $22 \cdot 65$ & $23 \cdot 9$ & $26 \cdot 7$ & 15 & 17 & $3+17$ & $3+13$ & 138 & vi & $1+33$ \\
\hline 680 & $1 \cdot 43$ & $4 \cdot 64$ & $11 \cdot 5$ & $12 \cdot 3$ & $14 \cdot 25$ & $17 \cdot 4$ & $22 \cdot 4$ & $23 \cdot 6$ & $26 \cdot 3$ & 14 & 17 & $3+16$ & $3+15$ & 162 & ii & $0+32$ \\
\hline 681 & $1 \cdot 38$ & $4 \cdot 4$ & $11 \cdot 0$ & $12 \cdot 0$ & $13 \cdot 95$ & $16 \cdot 75$ & $22 \cdot 05$ & $23 \cdot 3$ & $25 \cdot 9$ & 15 & 16 & $3+17$ & $4+14$ & 157 & vi & $2+32$ \\
\hline 682 & $1 \cdot 38$ & $4 \cdot 63$ & $11 \cdot 3$ & $12 \cdot 25$ & $14 \cdot 4$ & $17 \cdot 15$ & $22 \cdot 35$ & $23 \cdot 55$ & $26 \cdot 3$ & 15 & 17 & $3+17$ & $4+13$ & 126 & vii & $0+33$ \\
\hline 683 & $1 \cdot 46$ & $4 \cdot 75$ & $12 \cdot 3$ & $12 \cdot 6$ & $15 \cdot 25$ & $17 \cdot 45$ & $22 \cdot 9$ & $24 \cdot 2$ & $26 \cdot 9$ & 13 & 17 & $3+15$ & $4+14$ & 181 & $\mathrm{o}^{-1} \mathrm{v}-\mathrm{vi}$ & $1+32$ \\
\hline 684 & 1.52 & $4 \cdot 8$ & $12 \cdot 0$ & $13 \cdot 2$ & $14 \cdot 9$ & $18 \cdot 2$ & $23 \cdot 35$ & $24 \cdot 65$ & $27 \cdot 45$ & 14 & 17 & $3+16$ & $4+14$ & 161 & ô vi & $2+30$ \\
\hline 685 & $1 \cdot 46$ & $4 \cdot 65$ & $11 \cdot 75$ & $12 \cdot 5$ & $14 \cdot 4$ & $17 \cdot 55$ & $22 \cdot 45$ & $23 \cdot 7$ & $26 \cdot 5$ & 15 & 18 & $2+16$ & $3+14$ & 147 & ô v-vi & $\uparrow 0+32$ \\
\hline 686 & $1 \cdot 55$ & $4 \cdot 8$ & $11 \cdot 7$ & $12 \cdot 75$ & $14 \cdot 9$ & $17 \cdot 9$ & $23 \cdot 3$ & $24 \cdot 55$ & $27 \cdot 45$ & 15 & 16 & $3+17$ & $3+15$ & 161 & $\mathrm{v}$ & $1+32$ \\
\hline 687 & $1 \cdot 4$ & $4 \cdot 5$ & $11 \cdot 1$ & $12 \cdot 3$ & $13 \cdot 9$ & $17 \cdot 05$ & $22 \cdot 0$ & $23 \cdot 25$ & $25 \cdot 75$ & 15 & 17 & $3+16$ & $4+13$ & 125 & $\mathrm{o}^{0} \mathrm{iv}-\mathrm{v}$ & " $1+31$ \\
\hline 688 & $1 \cdot 65$ & $4 \cdot 9$ & $11 \cdot 55$ & $12 \cdot 75$ & $14 \cdot 3$ & $17 \cdot 7$ & $22 \cdot 8$ & $24 \cdot 0$ & $26 \cdot 6$ & 16 & 17 & $3+16$ & $4+13$ & 136 & o iv-v & $2+32$ \\
\hline 689 & $1 \cdot 45$ & $4 \cdot 6$ & $11 \cdot 45$ & $13 \cdot 05$ & $14 \cdot 3$ & $17 \cdot 85$ & $23 \cdot 2$ & $24 \cdot 45$ & $27 \cdot 1$ & 14 & 16 & $3+17$ & $3+14$ & 166 & $\hat{~} v^{2} \mathrm{i}$ & $1+33$ \\
\hline 690 & $1 \cdot 5$ & $4 \cdot 75$ & $11 \cdot 65$ & $12 \cdot 5$ & $14 \cdot 65$ & $17 \cdot 6$ & $22 \cdot 85$ & $24 \cdot 1$ & $26 \cdot 8$ & 15 & 16 & $3+16$ & $4+15$ & 146 & 우 vi-iii & $1+33$ \\
\hline 691 & $1 \cdot 3$ & $4 \cdot 15$ & $10 \cdot 1$ & $10 \cdot 9$ & $12 \cdot 7$ & $15 \cdot 4$ & $20 \cdot 2$ & $21 \cdot 2$ & $23 \cdot 65$ & 16 & 18 & $3+18$ & $3+15$ & 98 & 우 vii & $0+32$ \\
\hline 692 & $1 \cdot 45$ & $4 \cdot 52$ & $11 \cdot 3$ & $12 \cdot 4$ & $14 \cdot 2$ & $17 \cdot 2$ & $22 \cdot 4$ & $23 \cdot 65$ & $26 \cdot 2$ & 15 & 17 & $3+17$ & $3+15$ & 142 & $\hat{\mathrm{j}} \mathrm{vi}$ & $2+32$ \\
\hline 693 & $1 \cdot 47$ & $4 \cdot 6$ & $11 \cdot 2$ & $12 \cdot 5$ & $13 \cdot 9$ & $17 \cdot 15$ & $21 \cdot 8$ & $23 \cdot 05$ & $25 \cdot 7$ & 15 & 17 & $4+16$ & $3+13$ & 146 & $\mathrm{v}$ & $1+33$ \\
\hline 694 & $1 \cdot 5$ & $4 \cdot 65$ & $11 \cdot 4$ & $12 \cdot 4$ & $14 \cdot 05$ & $17 \cdot 2$ & $22 \cdot 5$ & $23 \cdot 65$ & $26 \cdot 35$ & 15 & 17 & $3+17$ & $3+14$ & 139 & ovi & $2+32$ \\
\hline 695 & $1 \cdot 48$ & $4 \cdot 55$ & $11 \cdot 4$ & $12 \cdot 1$ & $14 \cdot 35$ & $17 \cdot 05$ & $22 \cdot 15$ & $23 \cdot 25$ & $25 \cdot 75$ & 15 & 16 & $3+17$ & $3+14$ & 140 & vi-iv & $2+31$ \\
\hline 696 & $1 \cdot 34$ & $4 \cdot 4$ & 10.9 & $11 \cdot 85$ & 13.55 & $16 \cdot 7$ & $21 \cdot 85$ & $23 \cdot 0$ & $25 \cdot 5^{*}$ & 15 & 17 & $3+16$ & $3+14$ & 125 & vi & $1+32$ \\
\hline 697 & $1 \cdot 46$ & $4 \cdot 7$ & $11 \cdot 85$ & $12 \cdot 4$ & $14 \cdot 65$ & $17 \cdot 45$ & 22.55 & $23 \cdot 7$ & $26 \cdot 4$ & 14 & 17 & $3+16$ & $2+16$ & 125 & i-iii & $0+32$ \\
\hline
\end{tabular}




\begin{tabular}{|c|c|c|c|c|c|c|c|c|c|c|c|c|c|c|c|c|c|}
\hline $\begin{array}{l}\text { No. of } \\
\text { fish. }\end{array}$ & 1 & 2 & 3 & 4 & 5 & 6 & 7 & 8 & 9 & 11 & 12 & 13 & 14 & 15 & 16 & 17 & 18 \\
\hline 698 & $1 \cdot 42$ & $4 \cdot 93$ & $12 \cdot 6$ & $13 \cdot 7$ & $15 \cdot 3$ & $18 \cdot 9$ & $24 \cdot 3$ & $25 \cdot 7$ & $28 \cdot 1^{*}$ & 14 & 17 & $\begin{array}{c}\text { Damaged. } \\
3+13\end{array}$ & $4+13$ & 156 & ô vi & $2+31$ & 55 \\
\hline 699 & $1 \cdot 46$ & $4 \cdot 7$ & $11 \cdot 25$ & $12 \cdot 75$ & $14 \cdot 3$ & $17 \cdot 65$ & $22 \cdot 8$ & $24 \cdot 0$ & $26 \cdot 7$ & 15 & 16 & $2+16$ & $3+13$ & 143 & 우 vi & $\uparrow 0+31$ & 55 \\
\hline 700 & $1 \cdot 6$ & $4 \cdot 9$ & $11 \cdot 9$ & $13 \cdot 0$ & $15 \cdot 2$ & $18 \cdot 2$ & $23 \cdot 25$ & $24 \cdot 45$ & $27 \cdot 25$ & 16 & 16 & $3+17$ & $3+14$ & 149 & $\hat{\delta} \mathrm{v}-\mathrm{vi}$ & $2+32$ & 56 \\
\hline 701 & $1 \cdot 5$ & $4 \cdot 55$ & $11 \cdot 75$ & $12 \cdot 45$ & $14 \cdot 55$ & $17 \cdot 4$ & $22 \cdot 65$ & $23 \cdot 85$ & $26 \cdot 5$ & 15 & 16 & $3+16$ & $2+14$ & 149 & ô vi & $1+32$ & 56 \\
\hline 702 & $1 \cdot 7$ & $5 \cdot 45$ & $13 \cdot 6$ & $14 \cdot 1$ & $16 \cdot 7$ & $19 \cdot 7$ & $25 \cdot 7$ & $27 \cdot 15$ & $30 \cdot 15$ & 14 & 17 & $3+16$ & $3+15$ & 215 & 우 vi & $0+32$ & 55 \\
\hline 703 & 1.75 & $5 \cdot 55$ & $12 \cdot 9$ & $13 \cdot 7$ & $16 \cdot 0$ & $18 \cdot 8$ & $24 \cdot 4$ & $25 \cdot 7$ & $28 \cdot 6$ & 15 & 16 & $3+16$ & $3+14$ & 142 & ô vi-iii & $1+32$ & 55 \\
\hline 704 & $1 \cdot 5$ & $4 \cdot 9$ & $12 \cdot 3$ & $13 \cdot 25$ & $15 \cdot 3$ & $18 \cdot 0$ & $23 \cdot 6$ & $24 \cdot 9$ & $27 \cdot 75$ & 14 & 18 & $3+16$ & $4+13$ & 144 & $\sigma^{\prime} \mathrm{v}$ & $0+32$ & 56 \\
\hline 705 & $1 \cdot 52$ & $4 \cdot 92$ & $12 \cdot 45$ & $13 \cdot 1$ & $15 \cdot 6$ & $18 \cdot 6$ & $24 \cdot 25$ & $25 \cdot 6$ & $28 \cdot 4$ & 15 & 17 & $3+16$ & $3+16$ & 185 & 우 vi & $0+33$ & 56 \\
\hline 706 & $1 \cdot 6$ & $4 \cdot 8$ & $11 \cdot 8$ & $12 \cdot 7$ & $14 \cdot 5$ & $17 \cdot 75$ & $22 \cdot 9$ & $24 \cdot 15$ & $26 \cdot 6$ & 15 & 18 & $3+16$ & $3+14$ & 143 & $\hat{o}$ vi-iv & $1+32$ & 56 \\
\hline 707 & $1 \cdot 43$ & $4 \cdot 38$ & $11 \cdot 15$ & $11 \cdot 65$ & $13 \cdot 85$ & $16 \cdot 35$ & $21 \cdot 6$ & $22 \cdot 75$ & $25 \cdot 3$ & 14 & 18 & $3+16$ & $3+15$ & 136 & $\hat{\delta} \mathrm{v}$ & $1+33$ & 56 \\
\hline 708 & $1 \cdot 5$ & $4 \cdot 9$ & $12 \cdot 0$ & $12 \cdot 9$ & $14 \cdot 8$ & $17 \cdot 9$ & $23 \cdot 3$ & $24 \cdot 5$ & $27 \cdot 2$ & 15 & 16 & $3+16$ & $3+15$ & 150 & $\delta^{2} \mathrm{v}-\mathrm{vi}$ & $1+33$ & 56 \\
\hline 709 & $1 \cdot 7$ & $5 \cdot 33$ & $12 \cdot 8$ & 13.7 & $15 \cdot 9$ & 18.7 & $24 \cdot 6$ & $25 \cdot 9$ & $28 \cdot 8$ & 14 & 17 & $3+17$ & $3+15$ & 199 & $\widehat{\delta}$ vi & $1+32^{*}$ & 56 \\
\hline 710 & $1 \cdot 52$ & $4 \cdot 8$ & $12 \cdot 4$ & $13 \cdot 2$ & $15 \cdot 55$ & $18 \cdot 65$ & 23.95 & $25 \cdot 15$ & $27 \cdot 85$ & 15 & 18 & $3+16$ & $3+13$ & 201 & 우 vi & $2+32$ & 56 \\
\hline 711 & $1 \cdot 43$ & $4 \cdot 35$ & $11 \cdot 6$ & $12 \cdot 3$ & $14 \cdot 4$ & $17 \cdot 35$ & $22 \cdot 55$ & $23 \cdot 7$ & $26 \cdot 45$ & 16 & 17 & $3+16$ & $3+14$ & 158 & ô vi & $0+33$ & 55 \\
\hline 712 & $1 \cdot 4$ & $4 \cdot 4$ & $11 \cdot 1$ & $11 \cdot 95$ & $13 \cdot 8$ & $16 \cdot 7$ & $21 \cdot 4$ & $22 \cdot 55$ & $25 \cdot 05$ & 16 & 18 & $3+16$ & $3+14$ & 129 & ô vi & $2+31$ & 56 \\
\hline 713 & 1.5 & $4 \cdot 8$ & $11 \cdot 75$ & $12 \cdot 6$ & $14 \cdot 65$ & $17 \cdot 4$ & $22 \cdot 85$ & $24 \cdot 1$ & $26 \cdot 8$ & 15 & 17 & $2+17$ & $3+15$ & 148 & ô vi & $2+32$ & 56 \\
\hline 714 & $1 \cdot 6$ & $5 \cdot 0$ & $12 \cdot 2$ & $12 \cdot 9$ & $15 \cdot 2$ & $18 \cdot 1$ & $23 \cdot 45$ & $24 \cdot 8$ & $27 \cdot 4^{*}$ & 14 & 16 & $3+16$ & $3+15$ & 185 & ô vi & $2+31$ & 55 \\
\hline 715 & $1 \cdot 4$ & $4 \cdot 42$ & $10 \cdot 95$ & $12 \cdot 1$ & $13 \cdot 7$ & $16 \cdot 95$ & $22 \cdot 1$ & $23 \cdot 3$ & $25 \cdot 85$ & 15 & 17 & $3+16$ & $3+14$ & 153 & ô vi & $0+33$ & 56 \\
\hline 716 & $1 \cdot 52$ & $4 \cdot 7$ & $11 \cdot 65$ & $12 \cdot 4$ & $14 \cdot 45$ & $17 \cdot 65$ & $22 \cdot 8$ & $24 \cdot 1$ & $26 \cdot 9$ & 15 & 16 & $3+16$ & $3+16$ & 151 & 우 vi & $1+32$ & 56 \\
\hline 717 & $1 \cdot 6$ & $4 \cdot 82$ & $11 \cdot 75$ & $12 \cdot 8$ & $14 \cdot 75$ & $17 \cdot 8$ & $23 \cdot 2$ & $24 \cdot 5$ & $27 \cdot 0$ & 15 & 17 & $3+16$ & $3+14$ & 151 & ô iii-iv & $0+33$ & 56 \\
\hline 718 & $1 \cdot 45$ & $4 \cdot 8$ & $11 \cdot 7$ & $12 \cdot 35$ & $14 \cdot 5$ & $17 \cdot 2$ & $22 \cdot 65$ & $23 \cdot 85$ & $26 \cdot 45$ & 16 & 17 & $3+16$ & $3+14$ & 142 & $\sigma^{\prime} v-v i$ & $1+32$ & 56 \\
\hline 719 & $1 \cdot 6$ & $4 \cdot 6$ & $11 \cdot 6$ & $12 \cdot 3$ & $14 \cdot 3$ & $17 \cdot 3$ & $22 \cdot 5$ & $23 \cdot 7$ & $26 \cdot 2$ & 15 & 16 & $3+17$ & $3+14$ & 129 & 우 vi-iii & $1+32$ & 56 \\
\hline 720 & $1 \cdot 4$ & $4 \cdot 35$ & $10 \cdot 75$ & $11 \cdot 9$ & $13 \cdot 45$ & $16 \cdot 55$ & $21 \cdot 55$ & $22 \cdot 65$ & $25 \cdot 25$ & 14 & 16 & $3+16$ & $3+15$ & 131 & $\hat{o} \mathrm{v}$ & $\uparrow 2+32$ & 56 \\
\hline 721 & $1 \cdot 47$ & $4 \cdot 85$ & $11 \cdot 6$ & $12 \cdot 2$ & $14 \cdot 35$ & $17 \cdot 0$ & $22 \cdot 45$ & $23 \cdot 6$ & $26 \cdot 2$ & 14 & 17 & $3+16$ & $3+16$ & 140 & ôvi & $1+33$ & 56 \\
\hline 722 & $1 \cdot 48$ & $4 \cdot 66$ & $11 \cdot 6$ & $12 \cdot 9$ & $14 \cdot 45$ & $17 \cdot 8$ & $22 \cdot 95$ & $24 \cdot 2$ & $26 \cdot 95$ & 14 & 16 & $3+16$ & $3+15$ & 161 & ô vi & $2+32$ & 56 \\
\hline 723 & 1.45 & $4 \cdot 55$ & $11 \cdot 3$ & $12 \cdot 25$ & $14 \cdot 2$ & $17 \cdot 0$ & $22 \cdot 1$ & $23 \cdot 25$ & $26 \cdot 0$ & 15 & 16 & $3+16$ & $3+14$ & 142 & of vi-iv & $1+32$ & 56 \\
\hline 724 & $1 \cdot 57$ & $4 \cdot 7$ & $11 \cdot 5$ & $12 \cdot 45$ & $14 \cdot 05$ & $17 \cdot 1$ & $22 \cdot 3$ & 23.55 & $26 \cdot 3$ & 12 & 17 & $2+16$ & $3+15$ & 143 & ô iii & $1+31$ & 55 \\
\hline 725 & $1 \cdot 67$ & $5 \cdot 05$ & $12 \cdot 45$ & $13 \cdot 5$ & $15 \cdot 35$ & $18 \cdot 8$ & $23 \cdot 9$ & $25 \cdot 2$ & $28 \cdot 1$ & 15 & 17 & $3+16$ & $3+14$ & 168 & 字 $\mathbf{v}$ & $0+33$ & 55 \\
\hline 726 & 1.5 & $4 \cdot 85$ & $12 \cdot 05$ & $12 \cdot 55$ & $14 \cdot 85$ & $17 \cdot 4$ & $22 \cdot 7$ & $23 \cdot 9$ & $26 \cdot 6$ & 14 & 16 & $3+17$ & $\begin{array}{c}17 \\
+14 \text { probably.) }\end{array}$ & 138 & ô & $1+32$ & 56 \\
\hline 727 & $1 \cdot 46$ & $4 \cdot 54$ & $11 \cdot 6$ & $12 \cdot 2$ & $14 \cdot 4$ & $17 \cdot 3$ & $22 \cdot 15$ & $23 \cdot 35$ & $25 \cdot 9$ & 15 & 18 & $3+16$ & $4+13$ & 144 & 오 iv-v & $0+33$ & 56 \\
\hline 728 & 1.47 & $4 \cdot 8$ & $11 \cdot 8$ & $12 \cdot 9$ & $14 \cdot 7$ & $17 \cdot 8$ & $22 \cdot 8$ & $24 \cdot 0$ & $26 \cdot 8$ & 15 & 18 & $3+16$ & $3+14$ & 154 & ô vi & $0+34$ & 56 \\
\hline 729 & 1.5 & $4 \cdot 68$ & $11 \cdot 45$ & $11 \cdot 9$ & $14 \cdot 2$ & $17 \cdot 4$ & $22 \cdot 4$ & $23 \cdot 65$ & $26 \cdot 25$ & 14 & 16 & $3+15$ & $3+13$ & 139 & 우 vi-iii & $1+32$ & 56 \\
\hline 730 & $1 \cdot 52$ & $4 \cdot 76$ & $11 \cdot 7$ & $12 \cdot 35$ & 14.5 & $17 \cdot 25$ & $22 \cdot 7$ & $23 \cdot 9$ & $26 \cdot 5$ & 14 & 17 & $3+16$ & $1+3+14$ & 143 & $\hat{\sigma} \mathrm{v}$ & $2+32$ & 56 \\
\hline 731 & 1.5 & $4 \cdot 95$ & $11 \cdot 85$ & $12 \cdot 65$ & $14 \cdot 8$ & $17 \cdot 4$ & $22 \cdot 8$ & $24 \cdot 05$ & $26 \cdot 8$ & 13 & 18 & $3+17$ & $3+14$ & 154 & ô vi & $2+31$ & 56 \\
\hline 732 & $1 \cdot 78$ & $5 \cdot 42$ & $13 \cdot 4$ & $14 \cdot 1$ & $16 \cdot 35$ & $19 \cdot 7$ & $25 \cdot 55$ & $26 \cdot 95$ & $29 \cdot 6^{*}$ & 15 & 17 & $3+16$ & $3+14$ & 185 & 우 vi-iv & $2+32$ & 56 \\
\hline 733 & $1 \cdot 73$ & $5 \cdot 4$ & 13.05 & $13 \cdot 85$ & $16 \cdot 4$ & $19 \cdot 2$ & $24 \cdot 85$ & $26 \cdot 15$ & $29 \cdot 15$ & 14 & 17 & $3+17$ & $1+3+13$ & 179 & ô vi & $1+32$ & 55 \\
\hline 734 & 1.5 & $4 \cdot 8$ & $12 \cdot 0$ & 12.95 & $15 \cdot 15$ & $18 \cdot 1$ & $23 \cdot 3$ & $24 \cdot 6$ & $27 \cdot 4$ & 15 & 17 & $2+17$ & $3+14$ & 179 & 우 vi & $1+31$ & 55 \\
\hline
\end{tabular}




\begin{tabular}{|c|c|c|c|c|c|c|c|c|c|c|c|c|c|c|c|c|c|c|}
\hline 735 & $1 \cdot 5$ & $4 \cdot 7$ & $11 \cdot 95$ & $12 \cdot 75$ & $15 \cdot 1$ & $18 \cdot 35$ & $23 \cdot 65$ & $24 \cdot 9$ & $27 \cdot 65$ & 16 & 17 & $4+16$ & $3+14$ & 182 & 우 vi & $1+32$ & 56 & \\
\hline 736 & $1 \cdot 45$ & $4 \cdot 32$ & $11 \cdot 35$ & $12 \cdot 4$ & $14 \cdot 15$ & $17 \cdot 25$ & $22 \cdot 15$ & $23 \cdot 4$ & $26 \cdot 0$ & 15 & 17 & $3+16$ & $3+15$ & 153 & ô vi & $\dagger 0+32$ & 56 & \\
\hline 737 & $1 \cdot 7$ & $5 \cdot 3$ & $13 \cdot 3$ & $14 \cdot 25$ & $16 \cdot 4$ & $19 \cdot 7$ & $25 \cdot 4$ & $26 \cdot 7$ & $29 \cdot 55$ & 14 & 17 & $3+16$ & $3+14$ & 164 & ơ vii & $2+31$ & 55 & \\
\hline 738 & $1 \cdot 7$ & $5 \cdot 3$ & $12 \cdot 95$ & $14 \cdot 1$ & $16 \cdot 4$ & $19 \cdot 35$ & $25 \cdot 3$ & $26 \cdot 8$ & $29 \cdot 9$ & 14 & 17 & $3+16$ & $3+14$ & 200 & ovi & $2+31$ & 56 & \\
\hline 739 & $1 \cdot 4$ & $4 \cdot 5$ & $11 \cdot 75$ & $11 \cdot 9$ & $14 \cdot 6$ & $17 \cdot 2$ & $22 \cdot 5$ & $23 \cdot 75$ & $26 \cdot 15$ & 15 & 18 & $3+16$ & $3+14$ & 144 & $0 \mathrm{v}$ & $1+31$ & 55 & \\
\hline 740 & $1 \cdot 4$ & $4 \cdot 88$ & $12 \cdot 2$ & $13 \cdot 15$ & $15 \cdot 35$ & $18 \cdot 15$ & $23 \cdot 65$ & 24.95 & $27 \cdot 3^{*}$ & 14 & 16 & $3+17$ & $3+14$ & 182 & $\delta$ v-vi & $0+33$ & $56^{*}$ & \\
\hline 741 & $1 \cdot 48$ & $4 \cdot 65$ & $12 \cdot 0$ & $12 \cdot 6$ & $14 \cdot 7$ & $17 \cdot 6$ & $22 \cdot 9$ & $24 \cdot 15$ & $26 \cdot 85$ & 14 & 17 & $3+16$ & $3+14$ & 143 & $\widehat{O} \mathrm{v}$ & $2+31$ & 56 & \\
\hline 742 & 1.5 & $4 \cdot 6$ & 11.55 & $12 \cdot 1$ & $14 \cdot 45$ & $17 \cdot 1$ & $22 \cdot 4$ & $23 \cdot 6$ & $26 \cdot 05$ & 15 & 16 & $3+16$ & $3+14$ & 158 & ơ vi & $1+33$ & 57 & \\
\hline 743 & $1 \cdot 4$ & $4 \cdot 4$ & $11 \cdot 25$ & $12 \cdot 05$ & $14 \cdot 05$ & $16 \cdot 9$ & $21 \cdot 9$ & $23 \cdot 1$ & $25 \cdot 65$ & 15 & 17 & $3+16$ & $4+12$ & 143 & vi & $0+32$ & 56 & \\
\hline 744 & 1.5 & $4 \cdot 95$ & $11 \cdot 85$ & $12 \cdot 7$ & $14 \cdot 6$ & $17 \cdot 7$ & $23 \cdot 25$ & $24 \cdot 45$ & $27 \cdot 15$ & 12 & 16 & $3+15$ & $3+14$ & 138 & 우-ii & $0+32$ & 55 & \\
\hline 745 & 1.5 & $4 \cdot 56$ & $11 \cdot 3$ & $12 \cdot 25$ & $14 \cdot 05$ & $17 \cdot 4$ & $22 \cdot 2$ & $23 \cdot 5$ & $25 \cdot 85$ & 15 & 17 & $3+16$ & $3+14$ & 150 & $07 \mathrm{v}$ & $2+33$ & 57 & T \\
\hline 746 & $1 \cdot 5$ & $4 \cdot 67$ & $11 \cdot 35$ & $12 \cdot 1$ & $14 \cdot 1$ & $17 \cdot 05$ & $22 \cdot 0$ & $23 \cdot 3$ & $25 \cdot 9$ & 15 & 16 & $3+16$ & $3+14$ & 138 & o iii & $2+31$ & $54^{*}$ & 茴 \\
\hline 747 & $1 \cdot 58$ & $4 \cdot 6$ & $11 \cdot 4$ & $12 \cdot 4$ & $13 \cdot 9$ & $17 \cdot 25$ & $22 \cdot 25$ & $23 \cdot 4$ & $25 \cdot 9$ & 14 & 17 & $3+16$ & $3+14$ & 155 & ô vi & $1+32$ & 55 & 极 \\
\hline 748 & $1 \cdot 34$ & $4 \cdot 35$ & $11 \cdot 25$ & $12 \cdot 0$ & $13 \cdot 75$ & $16 \cdot 5$ & $21 \cdot 4$ & $22 \cdot 4$ & $25 \cdot 0$ & 14 & 16 & $3+15$ & $3+13$ & 129 & ô vi & $1+31$ & 56 & 国 \\
\hline 749 & $1 \cdot 45$ & $4 \cdot 73$ & $12 \cdot 1$ & $12 \cdot 85$ & $15 \cdot 15$ & $18 \cdot 0$ & $23 \cdot 25$ & $24 \cdot 55$ & $27 \cdot 1$ & 15 & 16 & $3+16$ & $3+14$ & 155 & & $0+32$ & 55 & T్రి \\
\hline 750 & $1 \cdot 4$ & $4 \cdot 57$ & $11 \cdot 4$ & $12 \cdot 0$ & $14 \cdot 3$ & $17 \cdot 0$ & $22 \cdot 35$ & $23 \cdot 55$ & $26 \cdot 25$ & 15 & 17 & $3+16$ & $3+14$ & 168 & & $1+33$ & 56 & 2 \\
\hline 751 & $1 \cdot 58$ & $4 \cdot 9$ & $11 \cdot 65$ & $12 \cdot 9$ & $14 \cdot 7$ & $17 \cdot 9$ & $23 \cdot 0$ & $24 \cdot 3$ & $27 \cdot 05$ & 15 & 18 & $3+17$ & $3+14$ & 125 & vii & $0+33$ & 56 & tis \\
\hline 752 & $1 \cdot 74$ & $5 \cdot 1$ & $12 \cdot 15$ & $13 \cdot 1$ & $15 \cdot 05$ & $18 \cdot 4$ & $24 \cdot 1$ & $25 \cdot 4$ & $28 \cdot 2$ & 14 & 17 & $3+16$ & $3+15$ & 160 & vi-iv & $1+33$ & 56 & $n$ \\
\hline 753 & $1 \cdot 74$ & $4 \cdot 95$ & $12 \cdot 4$ & $13 \cdot 15$ & $15 \cdot 4$ & $18 \cdot 45$ & $23 \cdot 95$ & $25 \cdot 2$ & $28 \cdot 05$ & 15 & 16 & $3+16$ & $3+15$ & 192 & vi & $1+32$ & 56 & 을 \\
\hline 754 & $1 \cdot 74$ & $5 \cdot 3$ & $12 \cdot 6$ & $13 \cdot 4$ & $15 \cdot 35$ & $18 \cdot 25$ & $23 \cdot 65$ & $24 \cdot 95$ & $27 \cdot 85$ & 13 & 18 & $3+15$ & $3+14$ & 164 & $\mathrm{i}$ & $2+32$ & 56 & \\
\hline 755 & 1.55 & $4 \cdot 67$ & $11 \cdot 8$ & $12 \cdot 5$ & $14 \cdot 85$ & $17 \cdot 3$ & $22 \cdot 95$ & $24 \cdot 1$ & $26 \cdot 7$ & 15 & 17 & $3+17$ & $3+15$ & 153 & $v-v$ & $2+32$ & 56 & 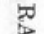 \\
\hline 756 & $1 \cdot 45$ & $4 \cdot 4$ & $11 \cdot 25$ & $12 \cdot 25$ & $14 \cdot 05$ & $16 \cdot 75$ & $21 \cdot 85$ & 22.95 & $25 \cdot 7$ & 15 & 17 & $3+16$ & $3+13$ & 158 & 아 vi & $\dagger 1+31$ & 55 & 2 \\
\hline 757 & 1.5 & $4 \cdot 64$ & $11 \cdot 5$ & $12 \cdot 4$ & $14 \cdot 35$ & $17 \cdot 05$ & $22 \cdot 2$ & $23 \cdot 35$ & $26 \cdot 0$ & 14 & 18 & $3+16$ & $1+2+16$ & 165 & & $2+32$ & 56 & 累 \\
\hline 758 & $1 \cdot 56$ & $4 \cdot 87$ & $11 \cdot 6$ & 12.55 & $14 \cdot 4$ & $17 \cdot 7$ & $22 \cdot 8$ & $23 \cdot 95$ & $26 \cdot 55$ & 14 & 18 & $3+17$ & $3+15$ & 148 & i & $1+33$ & 56 & \\
\hline 759 & $1 \cdot 5$ & $4 \cdot 75$ & $11 \cdot 85$ & $12 \cdot 7$ & $14 \cdot 6$ & $17 \cdot 45$ & $22 \cdot 85$ & $24 \cdot 15$ & $26 \cdot 45$ & 15 & 17 & $3+16$ & $3+15$ & 145 & & $2+32$ & 56 & 웟 \\
\hline 760 & $1 \cdot 55$ & $4 \cdot 88$ & $12 \cdot 25$ & $12 \cdot 65$ & $15 \cdot 0$ & $18 \cdot 6$ & $23 \cdot 4$ & $24 \cdot 7$ & $27 \cdot 2$ & 15 & 18 & $3+16$ & 17 rays broken. & 175 & & $0+33$ & 56 & \\
\hline 761 & $1 \cdot 38$ & $4 \cdot 2$ & $10 \cdot 6$ & $11 \cdot 4$ & $13 \cdot 15$ & $16 \cdot 15$ & $20 \cdot 7$ & $21 \cdot 9$ & $24 \cdot 6$ & 14 & 16 & $3+16$ & $3+13$ & 122 & $\mathrm{i}$ & $2+30$ & 55 & 正 \\
\hline 762 & 1.55 & $5 \cdot 0$ & $12 \cdot 3$ & $13 \cdot 2$ & $15 \cdot 3$ & $18 \cdot 6$ & $23 \cdot 9$ & $25 \cdot 15$ & $28 \cdot 05$ & 14 & 17 & $3+16$ & $3+14$ & 162 & & $1+31$ & 55 & $=$ \\
\hline 763 & $1 \cdot 45$ & $4 \cdot 5$ & $11 \cdot 35$ & $12 \cdot 4$ & $14 \cdot 1$ & $17 \cdot 3$ & $22 \cdot 4$ & $23 \cdot 55$ & $26 \cdot 4$ & 15 & 17 & $3+17$ & $3+13$ & 151 & 우 vi & $1+32$ & 55 & te=ㅂ \\
\hline 764 & $1 \cdot 43$ & $4 \cdot 5$ & $11 \cdot 7$ & $12 \cdot 3$ & $14 \cdot 4$ & $17 \cdot 45$ & $22 \cdot 7$ & $23 \cdot 95$ & $26 \cdot 6$ & 15 & 16 & $3+16$ & $3+14$ & 154 & & $+0+33$ & 56 & 呍 \\
\hline 765 & $1 \cdot 7$ & $5 \cdot 1$ & $12 \cdot 65$ & $13 \cdot 5$ & $15 \cdot 85$ & $18 \cdot 65$ & $24 \cdot 1$ & $25 \cdot 45$ & $28 \cdot 3$ & 16 & 17 & $3+17$ & $3+14$ & 179 & i & $1+33$ & 56 & $\frac{1}{2}$ \\
\hline 766 & $1 \cdot 64$ & $4 \cdot 9$ & $12 \cdot 5$ & $13 \cdot 0$ & $15 \cdot 35$ & $18 \cdot 55$ & $23 \cdot 7$ & $25 \cdot 05$ & $27 \cdot 9$ & 16 & 17 & $3+16$ & $3+14$ & 167 & & $1+33$ & 56 & \\
\hline 767 & $1 \cdot 7$ & $5 \cdot 1$ & $12 \cdot 3$ & $13 \cdot 9$ & $15 \cdot 5$ & $18 \cdot 8$ & $24 \cdot 3$ & $25 \cdot 6$ & $28 \cdot 3$ & 15 & 17 & $3+17$ & $1+14$ & 181 & vi & $2+32$ & $55^{*}$ & \\
\hline 768 & $1 \cdot 55$ & $4 \cdot 6$ & $11 \cdot 75$ & $13 \cdot 0$ & $14 \cdot 6$ & $17 \cdot 7$ & $23 \cdot 0$ & $24 \cdot 3$ & $26 \cdot 85$ & 14 & 17 & $3+15$ & $4+14$ & 151 & vi & $1+0+33$ & 56 & \\
\hline 769 & $1 \cdot 55$ & $4 \cdot 8$ & $11 \cdot 75$ & $12 \cdot 65$ & $14 \cdot 8$ & $18 \cdot 1$ & $23 \cdot 4$ & $24 \cdot 7$ & $27 \cdot 25$ & 14 & 16 & $3+17$ & $3+14$ & 162 & vi & $0+33$ & 55 & \\
\hline 770 & $1 \cdot 42$ & $4 \cdot 57$ & $11 \cdot 4$ & $12 \cdot 1$ & $14 \cdot 2$ & $17 \cdot 25$ & $22 \cdot 05$ & $23 \cdot 25$ & $25 \cdot 85$ & 16 & 17 & $3+16$ & $3+14$ & 151 & & $\dagger 0+32$ & 56 & \\
\hline 771 & $1 \cdot 6$ & $4 \cdot 64$ & $11 \cdot 75$ & $12 \cdot 5$ & $14 \cdot 45$ & $17 \cdot 5$ & $22 \cdot 9$ & $24 \cdot 2$ & $26 \cdot 95$ & 14 & 16 & $3+16$ & $3+15$ & 159 & 우 vi & $1+33$ & 56 & \\
\hline 772 & $1 \cdot 6$ & $4 \cdot 83$ & $11 \cdot 95$ & $12 \cdot 2$ & $14 \cdot 8$ & $17 \cdot 8$ & $22 \cdot 8$ & $24 \cdot 0$ & $26 \cdot 8$ & 16 & 16 & $3+17$ & $4+13$ & 162 & ô vi & $0+32$ & 55 & \\
\hline 773 & $1 \cdot 62$ & $4 \cdot 72$ & $11 \cdot 7$ & $12 \cdot 85$ & $14 \cdot 65$ & $17 \cdot 85$ & $23 \cdot 05$ & $24 \cdot 25$ & $26 \cdot 7$ & 15 & 16 & $3+16$ & $3+13$ & 160 & q iv-v & $0+33$ & 56 & $=$ \\
\hline 774 & 1.57 & $4 \cdot 66$ & $11 \cdot 6$ & $12 \cdot 25$ & $14 \cdot 2$ & $17 \cdot 25$ & $22 \cdot 5$ & $23 \cdot 8$ & $26 \cdot 3$ & 14 & 17 & $3+16$ & $3+14$ & 136 & & $+1+31$ & 55 & 8 \\
\hline 775 & $1 \cdot 56$ & $4 \cdot 78$ & $11 \cdot 4$ & $12 \cdot 7$ & $14 \cdot 1$ & $17 \cdot 3$ & $22 \cdot 55$ & $23 \cdot 75$ & $26 \cdot 3$ & 14 & 16 & $3+16$ & $3+13$ & 120 & vi-iii & $1+33$ & 56 & \\
\hline & -0 & & & 10.9 & $14 \cdot 6$ & $17 \cdot 55$ & $22 \cdot 7$ & 23.95 & $26 \cdot 4$ & 16 & 17 & $3+17$ & $3+15$ & 141 & iv & $2+32$ & 56 & \\
\hline
\end{tabular}




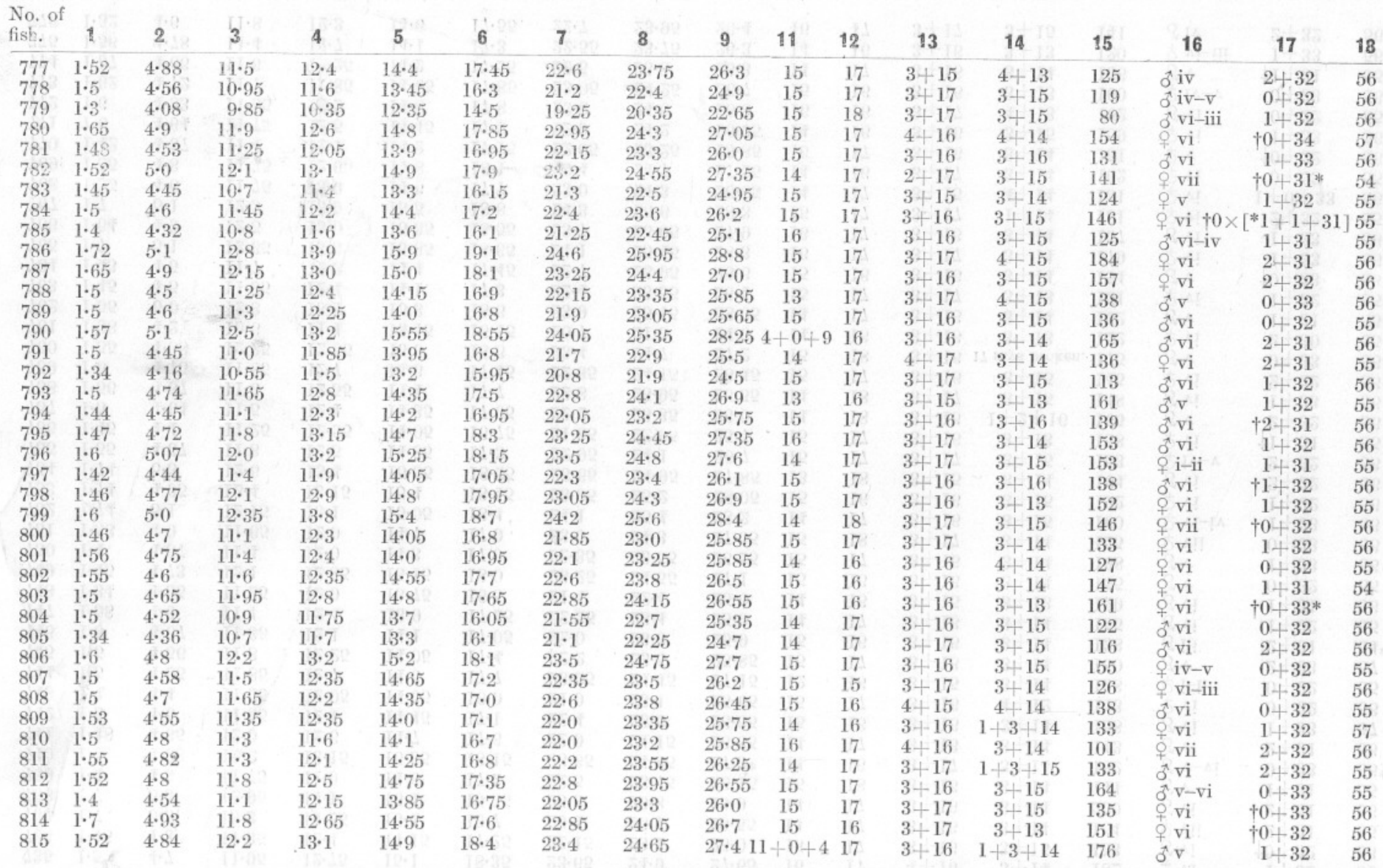


$816 \quad 1 \cdot 38$

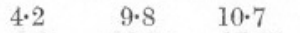
$9 \cdot$

818

$818 \quad 1 \cdot 6$

$\begin{array}{ll}819 & 1 \cdot 32 \\ 820 & 1.58\end{array}$

$821 \quad 1.5 \quad 5 \cdot 1$.

$822 \quad 1$

$\begin{array}{lll}823 & 1 \cdot 6 & 4 \cdot 95\end{array}$

$824 \quad 1 \cdot 47 \quad 4 \cdot 4$

$\begin{array}{lll}826 & 1 \cdot 34 & 4 \cdot 47\end{array}$

$827 \quad 1.5$

$\begin{array}{lll}828 & 1 \cdot 6 & 4 \cdot 65\end{array}$

$\begin{array}{lll}830 \quad 1.45 & 4.7\end{array}$

$831 \quad 1 \cdot 4$

$832 \quad 1$

$\begin{array}{ll}833 & 1 \cdot 5 \\ 834 & 1 \cdot 37\end{array}$

$835 \quad 1 \cdot 4 \quad 4 \cdot 38$

$836 \quad 1 \cdot$

$837 \quad 1 \cdot 45$

$838 \quad 1 \cdot 37$

$839 \quad 1.43$

$840 \quad 1$.

$841 \quad 1.7$

841.56

$845 \quad 1.45$

$846 \quad 1.5$

$847 \quad 1.56$

$848 \quad 1.5$

$850 \quad 1.4 \quad 4$.

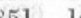

$\begin{array}{lll}852 & 1 \cdot 68\end{array}$

$853 \quad 1 \cdot 6$

$854 \quad 1 \cdot 52$

$\begin{array}{ll}855 & 1 \cdot 5 \\ 856 & 1 \cdot 4\end{array}$

$11 \cdot 6 \quad 12 \cdot 6$

$11 \cdot 1 \quad 12 \cdot 05$

$11.75 \quad 12.85$

$11 \cdot 15$

$4.1 \quad 10.1 \quad 11.1$

$10.8 ? \quad 11.5$

$11.05 \quad 11 \cdot 75$

$11 \cdot 65 \quad 12 \cdot 55$

$12 \cdot 3 \quad 12 \cdot 9$

$11 \cdot 73$

$11 \cdot 85 \quad 12 \cdot 8$

$13 \cdot 85$

$10 \cdot 75 \quad 11.55$

1.5513 .55

12.5

$\begin{array}{ll}12.55 & 13.0 \\ 11.05 & 11.65\end{array}$

$15 \cdot 5 \quad 18 \cdot 4$

12.4 .

14.85

$4 \cdot 9 \quad 11 \cdot 75$

$\begin{array}{lll}4.92 & 12.55 & 13.45\end{array}$

$4 \cdot 46$

$11 \cdot 55$

$12 \cdot 95$
$12 \cdot 25$

$15 \cdot 85 \quad 19 \cdot 05$

$\begin{array}{ll}14.55 & 17 \cdot 85 \\ 14.0 & 17 \cdot 15\end{array}$

$14 \cdot 6 \quad 17 \cdot 65$

$17 \cdot 4$

$11.7 \quad 12.55 \quad 14.6$

$12 \cdot 4$

$14 \cdot 2$

$2 \cdot 2 \quad 14 \cdot 85$

$19 \cdot 24$

$21 \cdot 85$

$23 \cdot 3$

오 vii

$+0+33$

ง vi-iv $\quad 0+33$

오 $\mathrm{i}-\mathrm{ii} \quad 1+33$

o vi $\quad 1+32$

vii $\quad+0+33$

아-iv $\quad 2+31$

ovi

ô vi $\quad+1+31$

ô vii $1+32$

ô vi $2+31$

vi $\quad 1+32$

우 vi $0+33$

ô $\mathrm{vi} \quad 2+31$

v $\quad+0+32$

우 $\quad \dagger 0+32$

$\begin{array}{ll}\text { ov } & 2+30 \\ \text { vi } & 1+32\end{array}$

ivi $3+31$

vi

의 vi

우 vii

ôvi

우 $\mathrm{v}$

oิ i-ii

우 vii.

ô vi

ovi

아 vi

우 vi-iv

ô $\mathrm{v}$

o vi $\quad 0+33$

ô $v-v i$

오 vi

ô vi

$1+33$

$4+30$

$0+32$
$3+31$ 


\begin{tabular}{|c|c|c|c|c|c|c|c|c|c|c|c|c|c|c|c|c|c|}
\hline $\begin{array}{l}\text { No. of } \\
\text { fish. }\end{array}$ & 1 & 2 & 3 & 4 & 5 & 6 & 7 & 8 & 9 & 11 & 12 & 13 & 14 & 15 & 16 & 17 & 18 \\
\hline 857 & $1 \cdot 47$ & $4 \cdot 95$ & $12 \cdot 4$ & $13 \cdot 45$ & $15 \cdot 15$ & $18 \cdot 45$ & $24 \cdot 15$ & $25 \cdot 4$ & $28 \cdot 15$ & $\begin{array}{c}\text { Fused. } \\
4+(1+1)+8 \\
=13 \text { or } 14 .\end{array}$ & 16 & $3+16$ & $3+14$ & 141 & 우 i-ii & $2+31$ & 56 \\
\hline 858 & $1 \cdot 68$ & $5 \cdot 03$ & $12 \cdot 05$ & $12 \cdot 55$ & 14.95 & $17 \cdot 4$ & $23 \cdot 05$ & $24 \cdot 3$ & $27 \cdot 15$ & 15 & 17 & $3+16$ & $3+14$ & 171 & 우 vi & $0+34$ & 56 \\
\hline 859 & $1 \cdot 63$ & $5 \cdot 07$ & $13 \cdot 05$ & $14 \cdot 2$ & $16 \cdot 1$ & $19 \cdot 75$ & $25 \cdot 3$ & $26 \cdot 6$ & $29 \cdot 55$ & 15 & 17 & $3+16$ & $3+14$ & 186 & ô vi-iv & $2+31$ & 56 \\
\hline 860 & $1 \cdot 62$ & $4 \cdot 9$ & $11 \cdot 9$ & $12 \cdot 95$ & $14 \cdot 75$ & $17 \cdot 9$ & $23 \cdot 3$ & $24 \cdot 65$ & $27 \cdot 5$ & 15 & 17 & $3+16$ & $3+15$ & 150 & 오 vi & $1+32$ & 56 \\
\hline 861 & 1.52 & $4 \cdot 62$ & $11 \cdot 7$ & $12 \cdot 75$ & $14 \cdot 5$ & $17 \cdot 9$ & $23 \cdot 1$ & $24 \cdot 3$ & $26 \cdot 9$ & 15 & 16 & $3+16$ & $1+3+14$ & 150 & 우 iv-v & $1+33$ & 56 \\
\hline 862 & 1.58 & $5 \cdot 0$ & $12 \cdot 55$ & $13 \cdot 2$ & $15 \cdot 25$ & $18 \cdot 4$ & $23 \cdot 9$ & $25 \cdot 3$ & $28 \cdot 05$ & 16 & 17 & $4+16$ & $3+14$ & 163 & ô vi & $2+31$ & 56 \\
\hline 863 & $1 \cdot 54$ & $4 \cdot 78$ & $11 \cdot 9$ & $12 \cdot 65$ & $14 \cdot 8$ & $17 \cdot 85$ & $23 \cdot 05$ & $24 \cdot 35$ & $27 \cdot 0$ & 14 & 17 & $3+17$ & $1+3+12$ & 138 & o vi-iii & $2+31$ & 55 \\
\hline 864 & 1.55 & $4 \cdot 43$ & $10 \cdot 95$ & $11 \cdot 8$ & $13 \cdot 4$ & $16 \cdot 4$ & $21 \cdot 2$ & $22 \cdot 4$ & $24 \cdot 8$ & 14 & 16 & $3+15$ & $3+14$ & 124 & $\hat{o} \mathrm{v}$ & $1+31$ & 55 \\
\hline 865 & $1 \cdot 47$ & $4 \cdot 5$ & $11 \cdot 35$ & $12 \cdot 45$ & $14 \cdot 3$ & $17 \cdot 5$ & $-22 \cdot 45$ & $23 \cdot 7$ & $26 \cdot 25$ & 15 & 17 & $3+16$ & $3+15$ & 156 & $0+\mathrm{v}$ & $1+32$ & 56 \\
\hline 866 & $1 \cdot 44$ & $4 \cdot 5$ & $11 \cdot 2$ & $11 \cdot 85$ & $14 \cdot 1$ & $16 \cdot 75$ & $21 \cdot 85$ & $23 \cdot 05$ & $25 \cdot 5$ & 15 & 17 & $4+16$ & $3+14$ & 123 & 우 vi & $1+32$ & 56 \\
\hline 867 & $1 \cdot 63$ & $4 \cdot 85$ & $11 \cdot 3$ & $11 \cdot 95$ & $14 \cdot 1$ & $16 \cdot 9$ & $22 \cdot 05$ & $23 \cdot 2$ & $25 \cdot 8$ & 14 & 17 & $3+16$ & $3+15$ & 124 & ô vii & $0+33$ & 56 \\
\hline 868 & $1 \cdot 63$ & $5 \cdot 07$ & $12 \cdot 1$ & $13 \cdot 35$ & $15 \cdot 0$ & $18 \cdot 35$ & $23 \cdot 95$ & $25 \cdot 35$ & $28 \cdot 15$ & 15 & 17 & $3+15$ & $3+14$ & 142 & 우 i-ii & $1+32$ & 56 \\
\hline 869 & $1 \cdot 5$ & $4 \cdot 66$ & $12 \cdot 15$ & $12 \cdot 75$ & $15 \cdot 1$ & $17 \cdot 95$ & $22 \cdot 9$ & $24 \cdot 3$ & $26 \cdot 85$ & 14 & 16 & $3+16$ & $1+3+14$ & 162 & $\partial^{\top} v$ & $0+32$ & 55 \\
\hline 870 & 1.55 & $4 \cdot 62$ & $12 \cdot 0$ & 12.95 & $14 \cdot 75$ & $17 \cdot 75$ & $23 \cdot 2$ & $24 \cdot 4$ & $27 \cdot 08$ & 15 & 17 & $3+16$ & $1+3+14$ & 164 & 우 v & $0+32$ & 56 \\
\hline 871 & $1 \cdot 74$ & $4 \cdot 9$ & $11 \cdot 8$ & $12 \cdot 7$ & $14 \cdot 85$ & $18 \cdot 0$ & $23 \cdot 35$ & $24 \cdot 7$ & $27 \cdot 5$ & 15 & 16 & $3+16$ & $2+16$ & 154 & ot vi & $0+32$ & 56 \\
\hline 872 & $1 \cdot 42$ & $4 \cdot 52$ & $11 \cdot 4$ & $12 \cdot 2$ & $14 \cdot 25$ & $17 \cdot 15$ & $22 \cdot 25$ & $23 \cdot 5$ & $26 \cdot 1$ & 15 & 17 & $3+16$ & $3+14$ & 126 & oิ vi-iv & $1+33$ & 56 \\
\hline 873 & $1 \cdot 37$ & $4 \cdot 36$ & $11 \cdot 35$ & $11 \cdot 9$ & $14 \cdot 3$ & $16 \cdot 95$ & $22 \cdot 2$ & $23 \cdot 45$ & $26 \cdot 05$ & 15 & 16 & $3+17$ & $3+16$ & 168 & o vi & $1+33$ & 57 \\
\hline 874 & $1 \cdot 6$ & $4 \cdot 8$ & $11 \cdot 8$ & $12 \cdot 2$ & $14 \cdot 55$ & $17 \cdot 4$ & $22 \cdot 4$ & $23 \cdot 75$ & $26 \cdot 25$ & 14 & 16 & $3+17$ & $3+13$ & 134 & $\sigma^{t} \mathbf{v}$ & $1+33$ & 56 \\
\hline 875 & $1 \cdot 5$ & $4 \cdot 85$ & $12 \cdot 1$ & $12 \cdot 95$ & $14 \cdot 95$ & $17 \cdot 85$ & $23 \cdot 15$ & $24 \cdot 4$ & $27 \cdot 1$ & 14 & 16 & $3+16$ & $3+14$ & 149 & 오 vi-iii & $1+32$ & 56 \\
\hline 876 & $1 \cdot 6$ & $4 \cdot 86$ & $12 \cdot 1$ & $12 \cdot 7$ & $15 \cdot 1$ & $17 \cdot 95$ & $23 \cdot 25$ & $24 \cdot 5$ & $27 \cdot 1$ & 15 & 17 & $2+17$ & $3+14$ & 175 & ơ vi & $1+31$ & 56 \\
\hline 877 & 1.5 & $4 \cdot 4$ & $11 \cdot 1$ & $12 \cdot 1$ & 13.95 & $16 \cdot 9$ & $22 \cdot 0$ & $23 \cdot 3$ & $26 \cdot 0$ & 16 & 16 & $3+16$ & $4+14$ & 150 & ô v-vi & $1+32$ & 55 \\
\hline 878 & $1 \cdot 35$ & $4 \cdot 2$ & $10 \cdot 4$ & $11 \cdot 25$ & $13 \cdot 0$ & $15 \cdot 6$ & $20 \cdot 4$ & $21 \cdot 5$ & $24 \cdot 0$ & 14 & 18 & $3+17$ & $3+14$ & 118 & o vi & $1+31$ & 54 \\
\hline 879 & $1 \cdot 52$ & $4 \cdot 7$ & $11 \cdot 55$ & $12 \cdot 15$ & $14 \cdot 4$ & $17 \cdot 1$ & $22 \cdot 3$ & $23 \cdot 5$ & $26 \cdot 2$ & 15 & 17 & $3+16$ & $3+15$ & 136 & ô vi & $2+32$ & 56 \\
\hline 880 & $1 \cdot 5$ & $4 \cdot 34$ & $10 \cdot 85$ & $12 \cdot 2$ & $13 \cdot 5$ & $16 \cdot 4$ & $21 \cdot 25$ & $22 \cdot 4$ & $24 \cdot 85$ & 14 & 17 & $3+16$ & $3+15$ & 128 & ô vi & $1+32$ & 56 \\
\hline 881 & $1 \cdot 65$ & $5 \cdot 2$ & $12 \cdot 7$ & $13 \cdot 35$ & $15 \cdot 6$ & $18 \cdot 85$ & $24 \cdot 3$ & $25 \cdot 55$ & $28 \cdot 5$ & 15 & 17 & $2+17$ & $3+14$ & 182 & ôvi & $0+33$ & 56 \\
\hline 882 & $1 \cdot 4$ & $4 \cdot 46$ & $11 \cdot 2$ & $12 \cdot 0$ & $14 \cdot 0$ & $16 \cdot 75$ & $21 \cdot 95$ & $23 \cdot 15$ & $25 \cdot 7$ & 14 & 17 & $3+16$ & $3+15$ & 127 & $\sigma^{t} v-v i$ & $0+33$ & 56 \\
\hline 883 & $1 \cdot 55$ & $5 \cdot 0$ & $12 \cdot 1$ & $13 \cdot 2$ & $15 \cdot 05$ & $18 \cdot 35$ & $23 \cdot 75$ & $25 \cdot 1$ & $27 \cdot 7$ & 15 & 18 & $3+16$ & $3+14$ & 164 & ô vi & $1+33$ & 57 \\
\hline 884 & $1 \cdot 6$ & $4 \cdot 7$ & $12 \cdot 1$ & $12 \cdot 7$ & $15 \cdot 0$ & $17 \cdot 7$ & $23 \cdot 05$ & $24 \cdot 35$ & $27 \cdot 0$ & 15 & 17 & $3+16$ & $2+14$ & 142 & 우 vi & $1+32$ & 56 \\
\hline 885 & $1 \cdot 57$ & $4 \cdot 7$ & $11 \cdot 85$ & $12 \cdot 65$ & $14 \cdot 55$ & $17 \cdot 9$ & $23 \cdot 0$ & $24 \cdot 25$ & $27 \cdot 1$ & $1+14$ & 16 & $3+16$ & $3+12$ & 155 & ô vi & $3+31$ & 55 \\
\hline 886 & 1.5 & $4 \cdot 64$ & $11 \cdot 25$ & $12 \cdot 0$ & $14 \cdot 15$ & $16 \cdot 95$ & $22 \cdot 1$ & $23 \cdot 2$ & $25 \cdot 75$ & 14 & 16 & $3+15$ & $4+13$ & 127 & $q \mathrm{i}-\mathrm{ii}$ & $0+32$ & 55 \\
\hline 887 & 1.45 & $4 \cdot 4$ & $10 \cdot 7$ & $11 \cdot 5$ & $13 \cdot 5$ & $16 \cdot 05$ & $20 \cdot 9$ & $22 \cdot 0$ & $24 \cdot 6$ & 14 & 17 & $3+17$ & $3+14$ & 119 & $\sigma^{A} \mathrm{v}$ & $2+31$ & 55 \\
\hline 888 & $1 \cdot 5$ & $4 \cdot 76$ & $11 \cdot 9$ & $12 \cdot 8$ & $14 \cdot 7$ & $17 \cdot 8$ & $22 \cdot 85$ & $24 \cdot 1$ & $26 \cdot 8$ & 15 & 18 & $3+16$ & $3+14$ & 135 & $\sigma^{*}$ vi-iii & $1+32$ & 56 \\
\hline 889 & $1 \cdot 57$ & $4 \cdot 8$ & $11 \cdot 7$ & $12 \cdot 7$ & $14 \cdot 25$ & $17 \cdot 7$ & $22 \cdot 85$ & $24 \cdot 0$ & $26 \cdot 55$ & 16 & 17 & $3+15$ & $2+15$ & 137 & ơ vi-iii & $0+33$ & 56 \\
\hline 890 & $1 \cdot 58$ & $4 \cdot 84$ & $11 \cdot 85$ & $13 \cdot 2$ & $14 \cdot 6$ & $18 \cdot 0$ & $23 \cdot 25$ & $24 \cdot 45$ & $27 \cdot 1$ & 13 & 17 & $3+15$ & $3+14$ & 155 & iv-v & $1+32$ & 56 \\
\hline 891 & $1 \cdot 6$ & $4 \cdot 9$ & $12 \cdot 0$ & $12 \cdot 8$ & $15 \cdot 05$ & $18 \cdot 2$ & $23 \cdot 45$ & $24 \cdot 75$ & $27 \cdot 4$ & 16 & 18 & $3+17$ & $3+14$ & 164 & i $\mathbf{v}$ & $1+34$ & 57 \\
\hline 892 & $1 \cdot 64$ & $4 \cdot 73$ & $11 \cdot 6$ & $12 \cdot 8$ & $14 \cdot 55$ & $17 \cdot 6$ & $22 \cdot 9$ & $24 \cdot 1$ & $26 \cdot 9$ & 15 & 16 & $3+17$ & $3+16$ & 151 & $\widehat{o}$ vi-iv & $0+33$ & 56 \\
\hline 893 & $1 \cdot 7$ & $5 \cdot 4$ & $13 \cdot 1$ & $14 \cdot 05$ & $16 \cdot 3$ & $19 \cdot 45$ & $25 \cdot 35$ & $26 \cdot 75$ & $29 \cdot 85$ & 15 & 18 & $3+16$ & $3+15$ & 173 & $\widehat{o}$ vii-ii & $2+31$ & 55 \\
\hline 894 & $1 \cdot 52$ & $4 \cdot 6$ & $11 \cdot 8$ & $12 \cdot 65$ & 14.55 & $17 \cdot 5$ & $23 \cdot 1$ & $24 \cdot 35$ & $26 \cdot 95$ & 15 & 16 & $3+16$ & $3+14$ & 157 & $i v-v$ & $2+32$ & 56 \\
\hline
\end{tabular}




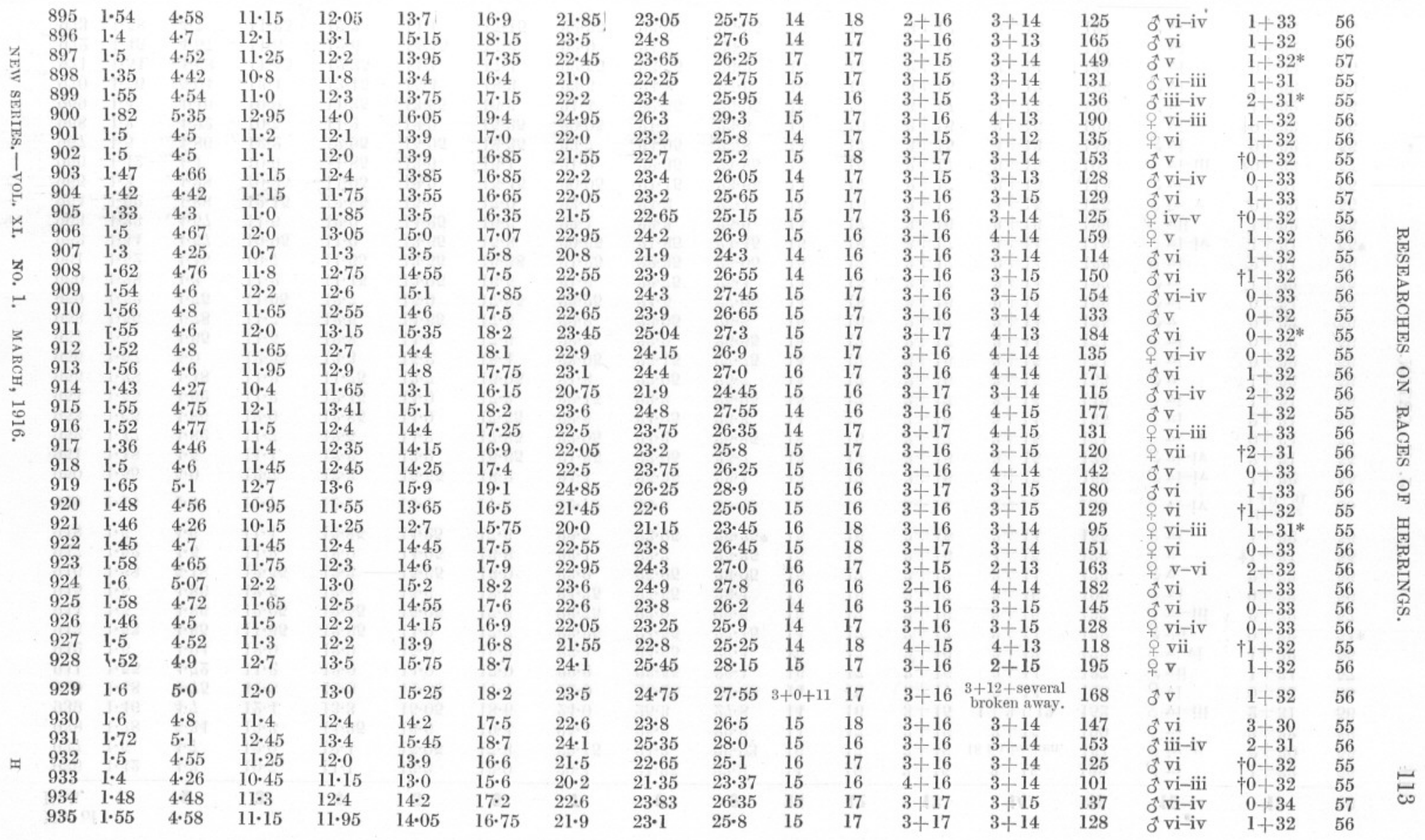




\begin{tabular}{|c|c|c|c|c|c|c|c|c|c|c|c|c|c|c|c|c|c|}
\hline $\begin{array}{l}\text { No. of } \\
\text { Fish. }\end{array}$ & 1 & 2 & 3 & 4 & 5 & 6 & 7 & 8 & 9 & 11 & 12 & 13 & 14 & 15 & 16 & 17 & 18 \\
\hline 936 & $1 \cdot 42$ & $4 \cdot 3$ & $10 \cdot 7$ & $11 \cdot 1$ & $13 \cdot 3$ & $16 \cdot 0$ & $20 \cdot 4$ & $21 \cdot 65$ & $23 \cdot 8$ & 14 & 16 & $3+15$ & $3+15$ & 122 & o vi & $1+32$ & 55 \\
\hline 937 & 1.5 & $4 \cdot 7$ & $12 \cdot 0$ & $12 \cdot 4$ & $15 \cdot 05$ & $17 \cdot 8$ & $23 \cdot 15$ & $24 \cdot 3$ & $26 \cdot 93$ & 16 & 17 & $3+16$ & 18 fin broken. & 161 & 우 $\mathbf{i v}-\mathbf{v}$ & $2+30$ & 55 \\
\hline 938 & $1 \cdot 78$ & $5 \cdot 34$ & $13 \cdot 3$ & $14 \cdot 35$ & $16 \cdot 6$ & $19 \cdot 4$ & $25 \cdot 4$ & $26 \cdot 8$ & $29 \cdot 8$ & 14 & 17 & $3+16$ & $3+15$ & 190 & 우 vi & $3+32$ & 56 \\
\hline 939 & $1 \cdot 46$ & $4 \cdot 7$ & $12 \cdot 4$ & $13 \cdot 3$ & $15 \cdot 05$ & $18 \cdot 6$ & $24 \cdot 0$ & $25 \cdot 3$ & $27 \cdot 8$ & 14 & 16 & $3+15$ & $1+3+13$ & 152 & 오 vi-iii & $2+31$ & 56 \\
\hline 940 & $1 \cdot 48$ & $4 \cdot 75$ & $11 \cdot 8$ & $12 \cdot 8$ & $14 \cdot 65$ & $17 \cdot 7$ & $22 \cdot 9$ & $24 \cdot 25$ & $26 \cdot 85$ & 14 & 18 & $3+17$ & $3+14$ & 146 & ơ vi & $0+32$ & 55 \\
\hline 941 & $1 \cdot 55$ & $4 \cdot 72$ & $11 \cdot 2$ & $12 \cdot 0$ & $14 \cdot 0$ & $17 \cdot 0$ & $22 \cdot 2$ & $23 \cdot 55$ & $26 \cdot 1$ & 16 & 17 & $3+16$ & $3+14$ & 137 & ô vii & $1+34$ & 57 \\
\hline 942 & $1 \cdot 6$ & $4 \cdot 94$ & $12 \cdot 0$ & $12 \cdot 9$ & $15 \cdot 0$ & $18 \cdot 3$ & $23 \cdot 4$ & $24 \cdot 65$ & $27 \cdot 4$ & 15 & 17 & $3+16$ & $3+13$ & 172 & q $\mathbf{v}$-vi & $1+32$ & 55 \\
\hline 943 & $1 \cdot 53$ & $4 \cdot 85$ & $11 \cdot 65$ & $12 \cdot 85$ & $14 \cdot 9$ & $17 \cdot 7$ & $23 \cdot 25$ & $24 \cdot 55$ & $27 \cdot 15$ & 14 & 17 & $3+17$ & $3+14$ & 138 & ot vii & $0+32$ & $54^{*} *$ \\
\hline 944 & 1.5 & $4 \cdot 86$ & $12 \cdot 05$ & $12 \cdot 55$ & $15 \cdot 05$ & $17 \cdot 6$ & $22 \cdot 75$ & $23 \cdot 9$ & $26 \cdot 5$ & 15 & 17 & $3+16$ & $3+16$ & 137 & o vi-iii & $2+32$ & 57 \\
\hline 945 & 1.5 & $4 \cdot 86$ & $12 \cdot 5$ & $13 \cdot 4$ & $15 \cdot 6$ & $18 \cdot 8$ & $24 \cdot 35$ & $25 \cdot 65$ & $28 \cdot 4$ & 14 & 16 & $3+17$ & $3+16$ & 199 & 우 vi & $0+32$ & 56 \\
\hline 946 & $1 \cdot 68$ & $5 \cdot 18$ & $12 \cdot 55$ & $13 \cdot 3$ & $15 \cdot 65$ & $18 \cdot 9$ & $23 \cdot 9$ & $25 \cdot 25$ & $28 \cdot 05$ & 15 & 17 & $3+17$ & $3+14$ & 176 & $\sigma^{t} \mathrm{v}$ & $2+33$ & 56 \\
\hline 947 & 1.52 & $4 \cdot 5$ & $11 \cdot 2$ & $12 \cdot 0$ & $14 \cdot 05$ & $16 \cdot 8$ & $22 \cdot 1$ & $23 \cdot 3$ & $25 \cdot 85$ & 15 & 17 & $3+16$ & $4+15$ & 138 & $\mathbf{v}$ & $+1+31$ & 55 \\
\hline 948 & $1 \cdot 5$ & $4 \cdot 6$ & $11 \cdot 4$ & $12 \cdot 85$ & $14 \cdot 25$ & $17 \cdot 6$ & $22 \cdot 7$ & $23 \cdot 95$ & $26 \cdot 25 *$ & 15 & 16 & $3+15$ & $3+14$ & 155 & 우 vi & $1+32$ & 56 \\
\hline 949 & $1 \cdot 46$ & $4 \cdot 6$ & $11 \cdot 7$ & $12 \cdot 1$ & $14 \cdot 4$ & $17 \cdot 3$ & $22 \cdot 73$ & $23 \cdot 9$ & $26 \cdot 45$ & 15 & 16 & $3+16$ & $4+14$ & 138 & 우 vi-iv & $\begin{array}{l}\text { Missing } \\
1+0+31\end{array}$ & 55 \\
\hline 950 & 1.55 & $5 \cdot 0$ & $12 \cdot 7$ & $13 \cdot 3$ & $15 \cdot 55$ & $18 \cdot 2$ & $24 \cdot 1$ & $25 \cdot 35$ & $28 \cdot 1$ & 13 & 17 & $3+16$ & $4+15$ & 168 & ơ vi-iv & $1+32$ & 55 \\
\hline 951 & $1 \cdot 48$ & $4 \cdot 5$ & $11 \cdot 1$ & $12 \cdot 2$ & $13 \cdot 9$ & $16 \cdot 65$ & $21 \cdot 85$ & $23 \cdot 05$ & $25 \cdot 7$ & 13 & 17 & $3+16$ & $3+15$ & 132 & ô vi-iv & $2+31$ & 55 \\
\hline 952 & $1 \cdot 47$ & $4 \cdot 82$ & $11 \cdot 7$ & $12 \cdot 5$ & $14 \cdot 8$ & $17 \cdot 85$ & $23 \cdot 05$ & $24 \cdot 2$ & $26 \cdot 85$ & 18 & 16 & $3+17$ & $3+15$ & 171 & oi & $0+34$ & 57 \\
\hline 953 & $1 \cdot 65$ & $4 \cdot 86$ & $12 \cdot 0$ & $12 \cdot 85$ & $14 \cdot 9$ & $17 \cdot 55$ & $22 \cdot 8$ & $24 \cdot 05$ & $26 \cdot 8$ & 14 & 17 & $3+16$ & $3+14$ & 135 & ㅇ vi & $2+31$ & 56 \\
\hline 954 & 1.55 & $4 \cdot 62$ & $11 \cdot 2$ & 12.45 & $14 \cdot 0$ & $17 \cdot 3$ & $22 \cdot 15$ & $23 \cdot 3$ & $25 \cdot 95$ & 15 & 17 & $3+17$ & $3+14$ & 149 & o vi & $1+31$ & 56 \\
\hline 955 & $1 \cdot 38$ & $4 \cdot 48$ & $11 \cdot 2$ & $11 \cdot 75$ & $13 \cdot 7$ & $16 \cdot 95$ & $21 \cdot 8$ & $23 \cdot 05$ & $25 \cdot 7$ & 14 & 17 & $2+16$ & $3+15$ & 153 & $\sigma^{t} \mathrm{v}$ & $1+33$ & 56 \\
\hline 956 & $1 \cdot 67$ & $5 \cdot 0$ & $12 \cdot 25$ & $13 \cdot 3$ & $15 \cdot 2$ & $18 \cdot 4$ & $23 \cdot 95$ & $25 \cdot 15$ & $27 \cdot 75$ & 14 & 17 & $3+16$ & $3+15$ & 171 & क्र vi & $0+32$ & 56 \\
\hline 957 & 1.5 & $4 \cdot 65$ & 11.5 & $12 \cdot 8$ & $14 \cdot 5$ & $17 \cdot 6$ & $22 \cdot 7$ & $23 \cdot 9$ & $26 \cdot 65$ & 14 & 17 & $2+17$ & $3+14$ & 171 & oi & $0+32$ & 56 \\
\hline 958 & $1 \cdot 56$ & $4 \cdot 78$ & $11 \cdot 7$ & $12 \cdot 6$ & $14 \cdot 65$ & $17 \cdot 5$ & $22 \cdot 8$ & $23 \cdot 95$ & $26 \cdot 6$ & 15 & 16 & $2+17$ & $3+14$ & 175 & $\hat{0} \mathbf{v}$ & $0+33$ & 56 \\
\hline 959 & $1 \cdot 52$ & $4 \cdot 75$ & $11 \cdot 95$ & $12 \cdot 7$ & $15 \cdot 0$ & $17 \cdot 85$ & $23 \cdot 25$ & $24 \cdot 5$ & $27 \cdot 05$ & 14 & 18 & $3+16$ & $2+14$ & 168 & ovi & $0+34$ & 56 \\
\hline 960 & $1 \cdot 38$ & $4 \cdot 3$ & $11 \cdot 2$ & $11 \cdot 45$ & 13.95 & $16 \cdot 5$ & $21 \cdot 6$ & $22 \cdot 75$ & $25 \cdot 4$ & 14 & 15 & $3+17$ & $3+14$ & 127 & ôvi & $1+32$ & 55 \\
\hline 961 & $1 \cdot 52$ & $4 \cdot 45$ & $10 \cdot 7$ & $11 \cdot 65$ & 13.55 & $16 \cdot 38$ & $21 \cdot 25$ & $22 \cdot 35$ & $25 \cdot 05$ & 15 & 17 & $3+17$ & $3+14$ & 132 & 우 vi & $2+31$ & 56 \\
\hline 962 & $1 \cdot 44$ & $4 \cdot 57$ & $10 \cdot 95$ & $11 \cdot 0$ & $13 \cdot 65$ & $15 \cdot 8$ & $20 \cdot 65$ & $21 \cdot 85$ & $24 \cdot 45$ & 15 & 18 & $3+17$ & $4+14$ & 116 & o vi-iv & $1+31$ & $55^{*}$ \\
\hline 963 & $1 \cdot 33$ & $4 \cdot 57$ & $11 \cdot 6$ & $12 \cdot 0$ & $14 \cdot 25$ & $16 \cdot 6$ & $22 \cdot 1$ & $23 \cdot 3$ & $25 \cdot 9$ & 14 & 17 & $3+16$ & $3+16$ & 128 & 우 vii & $1+32$ & 55 \\
\hline 964 & 1.62 & $4 \cdot 88$ & $12 \cdot 15$ & $12 \cdot 85$ & $15 \cdot 0$ & $17 \cdot 9$ & $23 \cdot 1$ & $24 \cdot 3$ & $27 \cdot 1$ & 16 & 16 & $3+17$ & $4+15$ & 155 & 우 iv-v & $0+32$ & 55 \\
\hline 965 & $1 \cdot 3$ & $4 \cdot 28$ & $10 \cdot 25$ & $11 \cdot 05$ & $12 \cdot 9$ & $15 \cdot 45$ & $20 \cdot 05$ & $21 \cdot 15$ & $23 \cdot 65$ & 14 & 17 & $2+17$ & $3+15$ & 118 & ô vi & $2+31$ & 55 \\
\hline 966 & $1 \cdot 42$ & $4 \cdot 4$ & $10 \cdot 9$ & $11 \cdot 85$ & 13.9 & $16 \cdot 1$ & $21 \cdot 2$ & $22 \cdot 35$ & $24 \cdot 85$ & 14 & 16 & $2+17$ & $3+15$ & 107 & o vi-iii & $\dagger 0+33$ & 57 \\
\hline 967 & 1.5 & $4 \cdot 86$ & $12 \cdot 2$ & 12.95 & $15 \cdot 05$ & $18 \cdot 35$ & $23 \cdot 7$ & 24.95 & $27 \cdot 8$ & 14 & 17 & $3+15$ & $4+13$ & 146 & ov vi & $0+32$ & 56 \\
\hline 968 & $1 \cdot 3$ & $4 \cdot 22$ & $10 \cdot 5$ & $11 \cdot 2$ & $12 \cdot 9$ & $15 \cdot 35$ & $20 \cdot 05$ & $21 \cdot 1$ & $23 \cdot 5$ & 14 & 16 & $3+16$ & $3+15$ & 106 & 우 vi & $+0+33$ & 56 \\
\hline 969 & 1.4 & $4 \cdot 46$ & $10 \cdot 9$ & $11 \cdot 35$ & $13 \cdot 65$ & $16 \cdot 6$ & $21 \cdot 4$ & $22 \cdot 55$ & $25 \cdot 05$ & 16 & 16 & $3+17$ & $3+15$ & 133 & ot vi & $1+32$ & 56 \\
\hline 970 & 1.56 & $5 \cdot 04$ & $12 \cdot 4$ & $13 \cdot 15$ & $15 \cdot 4$ & $18 \cdot 3$ & $23 \cdot 5$ & $24 \cdot 8$ & $27 \cdot 6$ & 16 & 17 & $3+15$ & $3+13$ & 158 & ôvi & $1+33$ & 56 \\
\hline 971 & 1.54 & $4 \cdot 68$ & 11.45 & $12 \cdot 35$ & $14 \cdot 1$ & $17 \cdot 4$ & $22 \cdot 55$ & $23 \cdot 75$ & $26 \cdot 2$ & 15 & 17 & $2+17$ & $3+14$ & 145 & o vi & $0+33$ & 55 \\
\hline 972 & 1.45 & $4 \cdot 57$ & $11 \cdot 6$ & $12 \cdot 6$ & $14 \cdot 45$ & $17 \cdot 6$ & $22 \cdot 9$ & $24 \cdot 1$ & $26 \cdot 7$ & 15 & 17 & $3+16$ & $3+16$ & 147 & 우 vi & $1+32$ & 55 \\
\hline 973 & $1 \cdot 58$ & $4 \cdot 7$ & $11 \cdot 25$ & $12 \cdot 3$ & $14 \cdot 1$ & $17 \cdot 15$ & $22 \cdot 35$ & $23 \cdot 6$ & $26 \cdot 25$ & 15 & 17 & $3+17$ & $3+16$ & 131 & ô vi & $0+33$ & 56 \\
\hline
\end{tabular}




\begin{tabular}{|c|c|c|c|c|c|c|c|c|c|c|c|c|c|c|c|c|c|c|}
\hline 974 & 1.5 & $4 \cdot 68$ & $11 \cdot 6$ & $12 \cdot 55$ & $14 \cdot 4$ & $17 \cdot 25$ & $22 \cdot 6$ & $23 \cdot 9$ & $26 \cdot 5$ & 14 & 17 & $3+16$ & $3+14$ & 136 & ㅇ vi & $1+32$ & 56 & \\
\hline 975 & $1 \cdot 35$ & $4 \cdot 35$ & $11 \cdot 0$ & $11 \cdot 5$ & $13 \cdot 65$ & $16 \cdot 6$ & $21 \cdot 5$ & $22 \cdot 65$ & $25 \cdot 15$ & 15 & 16 & $3+16$ & $3+14$ & 127 & or-vi & $0+31$ & $55^{*}$ & \\
\hline 976 & $1 \cdot 5$ & $4 \cdot 6$ & $11 \cdot 55$ & $12 \cdot 05$ & $14 \cdot 65$ & $17 \cdot 2$ & $22 \cdot 5$ & $24 \cdot 0$ & $26 \cdot 7$ & 14 & 16 & $3+17$ & $3+16$ & 136 & 우 vii & $1+32$ & 56 & \\
\hline 977 & $1 \cdot 48$ & $4 \cdot 63$ & $11 \cdot 0$ & $11 \cdot 65$ & $13 \cdot 75$ & $16 \cdot 3$ & $21 \cdot 1$ & $22 \cdot 28$ & $24 \cdot 7$ & 15 & 16 & $3+17$ & $3+13$ & 106 & 우 vi-iii & $1+32$ & 56 & \\
\hline 978 & $1 \cdot 4$ & $4 \cdot 4$ & $11 \cdot 05$ & $11 \cdot 65$ & $13 \cdot 8$ & $16 \cdot 25$ & $21 \cdot 4$ & $22 \cdot 5$ & $25 \cdot 1$ & 14 & 17 & $3+16$ & $3+15$ & 126 & 우 vi & $2+31$ & 56 & \\
\hline 979 & $1 \cdot 52$ & $4 \cdot 7$ & $11 \cdot 4$ & $12 \cdot 4$ & $14 \cdot 05$ & $17 \cdot 1$ & $22 \cdot 35$ & $23 \cdot 5$ & $26 \cdot 05$ & 14 & 17 & $3+15$ & $3+13$ & 137 & $\mathrm{o}^{*} \mathrm{v}$ & $2+31$ & 56 & \\
\hline 980 & $1 \cdot 52$ & $4 \cdot 66$ & $11 \cdot 75$ & $12 \cdot 65$ & $14 \cdot 8$ & $17 \cdot 5$ & $22 \cdot 8$ & $23 \cdot 95$ & $26 \cdot 7$ & 15 & 16 & $2+17$ & $3+13$ & 157 & $\sigma^{*} \mathrm{vi}$ & $0+33$ & $56 *$ & \\
\hline 981 & $1 \cdot 43$ & $4 \cdot 5$ & $11 \cdot 0$ & $11 \cdot 85$ & $13 \cdot 7$ & $16 \cdot 65$ & $21 \cdot 45$ & $22 \cdot 6$ & $25 \cdot 35$ & 15 & 16 & $3+16$ & $3+13$ & 143 & 우 vi & $0+32$ & 55 & \\
\hline 982 & $1 \cdot 57$ & $4 \cdot 77$ & $11 \cdot 35$ & $11 \cdot 55$ & $14 \cdot 2$ & $16 \cdot 45$ & $21 \cdot 95$ & $23 \cdot 2$ & $25 \cdot 8$ & 15 & 17 & $2+17$ & $3+14$ & 126 & ơ vii & $1+33$ & 57 & \\
\hline 983 & $1 \cdot 3$ & $4 \cdot 26$ & $10 \cdot 55$ & $11 \cdot 2$ & $13 \cdot 1$ & $15 \cdot 6$ & $20 \cdot 45$ & $21 \cdot 55$ & $24 \cdot 0$ & 15 & 18 & $3+15$ & $3+15$ & 103 & 우 vi-iv & $2+33$ & 57 & \\
\hline 984 & $1 \cdot 66$ & $5 \cdot 15$ & 12.55 & $13 \cdot 7$ & $15 \cdot 45$ & $18 \cdot 85$ & $24 \cdot 05$ & $25 \cdot 4$ & $28 \cdot 3$ & 14 & 16 & $3+15$ & $3+13$ & 152 & 우 i-ii & $2+30$ & 55 & \\
\hline 985 & $1 \cdot 42$ & $4 \cdot 72$ & $12 \cdot 0$ & $13 \cdot 2$ & $15 \cdot 2$ & $18 \cdot 65$ & $24 \cdot 05$ & $25 \cdot 3$ & $28 \cdot 35$ & 16 & 16 & $4+16$ & $3+14$ & 185 & 우 v-vi & $1+32$ & 56 & 果 \\
\hline 986 & $1 \cdot 53$ & $4 \cdot 4$ & $11 \cdot 4$ & $12 \cdot 0$ & $14 \cdot 0$ & $17 \cdot 0$ & $22 \cdot 1$ & $23 \cdot 25$ & $25 \cdot 9$ & 15 & 17 & $3+16$ & $3+14$ & 132 & ㅇ vi & $1+32$ & 56 & 팹 \\
\hline 987 & $1 \cdot 42$ & $4 \cdot 26$ & $10 \cdot 56$ & $11 \cdot 4$ & $13 \cdot 15$ & $16 \cdot 4$ & $21 \cdot 25$ & $22 \cdot 4$ & $24 \cdot 75$ & 16 & 17 & $3+16$ & $4+13$ & 117 & 우 iv-v & $1+33$ & 57 & 四 \\
\hline 988 & $1 \cdot 54$ & $4 \cdot 66$ & $11 \cdot 6$ & $12 \cdot 4$ & $14 \cdot 3$ & $17 \cdot 55$ & $22 \cdot 9$ & $24 \cdot 05$ & $26 \cdot 7$ & 15 & 16 & $3+15$ & $3+13$ & 145 & 오 vi & $\dagger 1+33$ & 57 & $d$ \\
\hline 989 & $1 \cdot 65$ & $5 \cdot 0$ & $12 \cdot 5$ & $13 \cdot 75$ & $15 \cdot 2$ & $18 \cdot 6$ & $24 \cdot 3$ & $25 \cdot 55$ & $28 \cdot 25$ & 15 & 18 & $3+16$ & $3+16$ & 156 & 우 vi & $2+32$ & 56 & 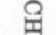 \\
\hline 990 & $1 \cdot 72$ & $5 \cdot 03$ & $12 \cdot 15$ & $13 \cdot 4$ & $15 \cdot 25$ & 18.55 & $23 \cdot 85$ & $25 \cdot 1$ & $27 \cdot 95$ & 15 & 16 & $3+17$ & $3+14$ & 171 & 우 vi & $1+32$ & 56 & 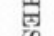 \\
\hline 991 & $1 \cdot 52$ & $4 \cdot 75$ & $11 \cdot 9$ & $12 \cdot 8$ & $14 \cdot 8$ & $18 \cdot 1$ & $23 \cdot 35$ & $24 \cdot 7$ & $27 \cdot 5^{*}$ & 14 & 17 & $3+16$ & $1+3+14$ & 171 & ơ vi & $0+32$ & 55 & 0 \\
\hline 992 & $1 \cdot 68$ & $5 \cdot 05$ & $12 \cdot 35$ & $13 \cdot 15$ & $15 \cdot 3$ & $18 \cdot 35$ & $24 \cdot 0$ & $25 \cdot 3$ & $28 \cdot 15$ & 14 & 17 & $3+16$ & $4+14$ & 147 & o i-ii & $2+31$ & 55 & tot \\
\hline 993 & $1 \cdot 47$ & $4 \cdot 67$ & $11 \cdot 45$ & $12 \cdot 4$ & $14 \cdot 15$ & $17 \cdot 65$ & $22 \cdot 6$ & $23 \cdot 85$ & $26 \cdot 45$ & 15 & 17 & $3+16$ & $3+13$ & 149 & iv-v & $1+33$ & 56 & $z$ \\
\hline 994 & $1 \cdot 48$ & $4 \cdot 53$ & $11 \cdot 0$ & $11 \cdot 6$ & $13 \cdot 95$ & $16 \cdot 4$ & $21 \cdot 45$ & $22 \cdot 6$ & $25 \cdot 05$ & 14 & 17 & $3+17$ & $4+14$ & 134 & ơ vi & $\dagger 0$ or $1+33$ & 56 & 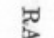 \\
\hline 995 & $1 \cdot 5$ & $4 \cdot 52$ & $11 \cdot 35$ & $12 \cdot 35$ & $14 \cdot 0$ & $17 \cdot 4$ & $22 \cdot 15$ & $23 \cdot 35$ & $25 \cdot 9$ & 14 & 17 & $3+16$ & 18 ray broken. & 123 & ơ vii & $2+32$ & 56 & \\
\hline 996 & $1 \cdot 65$ & $4 \cdot 96$ & $12 \cdot 6$ & $13 \cdot 45$ & $15 \cdot 6$ & $18 \cdot 9$ & $24 \cdot 15$ & $25 \cdot 5$ & $28 \cdot 4$ & 15 & $\begin{array}{l}\text { Fused. } \\
9+(1+1)+5\end{array}$ & ${ }_{5} 3+15$ & $4+14$ & 191 & 우 $\mathbf{v}$ & $1+31$ & 55 & 至 \\
\hline 997 & $1 \cdot 62$ & $4 \cdot 83$ & $12 \cdot 2$ & $12 \cdot 8$ & $14 \cdot 85$ & $18 \cdot 2$ & $23 \cdot 6$ & 24.95 & $27 \cdot 8$ & 15 & 16 & $3+16$ & $3+13$ & 164 & 우 vi & $1+32$ & 56 & 운 \\
\hline 998 & $1 \cdot 47$ & $4 \cdot 8$ & $11 \cdot 35$ & $12 \cdot 35$ & $14 \cdot 15$ & $17 \cdot 2$ & $22 \cdot 5$ & $23 \cdot 75$ & $26 \cdot 25$ & 15 & 16 & $3+17$ & $3+14$ & 124 & ơ vi-iv & $1+33$ & 57 & 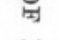 \\
\hline 999 & $1 \cdot 6$ & $4 \cdot 8$ & $12 \cdot 1$ & $12 \cdot 9$ & $15 \cdot 0$ & $18 \cdot 15$ & $23 \cdot 3$ & $24 \cdot 6$ & $27 \cdot 3$ & 14 & 17 & $2+15$ & $3+14$ & 173 & 우 vi & $1+33$ & 57 & \\
\hline 1000 & $1 \cdot 66$ & $4 \cdot 8$ & $11 \cdot 75$ & $12 \cdot 0$ & $14 \cdot 9$ & $17 \cdot 3$ & $22 \cdot 6$ & $23 \cdot 95$ & $26 \cdot 55$ & 15 & 17 & $3+16$ & $2+15$ & 143 & ơ $\mathrm{v}$ & $1+32$ & 54 & \\
\hline 1001 & $1 \cdot 4$ & $4 \cdot 58$ & $11 \cdot 74$ & $12 \cdot 6$ & $14 \cdot 65$ & $17 \cdot 95$ & $23 \cdot 05$ & $24 \cdot 35$ & $27 \cdot 0$ & 14 & 17 & $2+17$ & $3+15$ & 163 & 우 vi-iii & $1+32$ & 56 & ton \\
\hline 1002 & $1 \cdot 7$ & $5 \cdot 0$ & $12 \cdot 2$ & $12 \cdot 95$ & $15 \cdot 1$ & $17 \cdot 8$ & $23 \cdot 0$ & $24 \cdot 3$ & $26 \cdot 95$ & 14 & 16 & $3+16$ & $3+13$ & 157 & $\delta \mathrm{v}$ & $2+31$ & 56 & $y$ \\
\hline 1003 & $1 \cdot 52$ & $4 \cdot 97$ & $11 \cdot 85$ & $12 \cdot 55$ & $14 \cdot 5$ & $17 \cdot 4$ & $23 \cdot 05$ & $24 \cdot 4$ & $26 \cdot 95$ & 14 & 17 & $3+15$ & $3+14$ & 163 & ర vi & $1+32$ & 55 & \\
\hline 1004 & $1 \cdot 4$ & $4 \cdot 7$ & $11 \cdot 8$ & $12 \cdot 15$ & $14 \cdot 7$ & $17 \cdot 45$ & $22 \cdot 65$ & $23 \cdot 85$ & $26 \cdot 55$ & 14 & 17 & $3+16$ & $3+15$ & 152 & 우 $\mathbf{v}$ & $2+32$ & 56 & \\
\hline 1005 & $1 \cdot 9$ & $5 \cdot 74$ & $14 \cdot 1$ & $14 \cdot 77$ & $17 \cdot 45$ & $20 \cdot 6$ & $26 \cdot 7$ & $28 \cdot 1$ & $31 \cdot 2$ & 15 & 17 & $3+16$ & $3+14$ & 218 & ôvi & $2+31$ & 57 & \\
\hline 1006 & $1 \cdot 72$ & $5 \cdot 0$ & $12 \cdot 5$ & $13 \cdot 1$ & $15 \cdot 6$ & $18 \cdot 2$ & $23 \cdot 8$ & $25 \cdot 03$ & $27 \cdot 6$ & 15 & 16 & $3+16$ & $2+15$ & 175 & $\mathrm{o}^{1} \mathrm{vi}$ & $1+33$ & 56 & \\
\hline 1007 & $1 \cdot 6$ & $5 \cdot 0$ & $12 \cdot 65$ & $14 \cdot 0$ & $15 \cdot 75$ & $18 \cdot 9$ & $24 \cdot 35$ & $25 \cdot 7$ & $28 \cdot 6$ & 14 & 17 & $3+17$ & $3+14$ & 184 & 우 vi & $1+32$ & 56 & \\
\hline 1008 & $1 \cdot 6$ & $4 \cdot 9$ & $11 \cdot 95$ & $12 \cdot 5$ & $14 \cdot 8$ & $17 \cdot 65$ & $22 \cdot 8$ & $24 \cdot 1$ & $26 \cdot 9$ & 15 & 18 & $3+16$ & $3+14$ & 150 & ơ vi & $1+31$ & 55 & \\
\hline 1009 & $1 \cdot 65$ & $5 \cdot 0$ & $12 \cdot 0$ & $12 \cdot 55$ & $14 \cdot 9$ & $17 \cdot 55$ & $22 \cdot 9$ & $24 \cdot 2$ & $27 \cdot 1$ & 15 & 18 & $3+17$ & $3+15$ & 159 & 우 vi & $1+32$ & 56 & \\
\hline 1010 & $1 \cdot 4$ & $4 \cdot 6$ & $11 \cdot 7$ & $12 \cdot 2$ & $14 \cdot 25$ & $17 \cdot 4$ & $22 \cdot 7$ & $23 \cdot 85$ & $26 \cdot 5$ & 16 & 16 & $3+15$ & $3+14$ & 154 & ôvi & $3+30$ & $55^{*}$ & \\
\hline 1011 & $1 \cdot 55$ & $5 \cdot 12$ & $12 \cdot 6$ & 13.45 & $15 \cdot 45$ & $18 \cdot 5$ & $24 \cdot 0$ & $25 \cdot 35$ & $28 \cdot 2$ & 14 & 17 & $3+16$ & $3+15$ & 159 & ơ vi-iii & $0+33$ & 56 & \\
\hline 1012 & $1 \cdot 5$ & $4 \cdot 77$ & $11 \cdot 4$ & $12 \cdot 65$ & $14 \cdot 05$ & $17 \cdot 4$ & $22 \cdot 2$ & $23 \cdot 35$ & $26 \cdot 05$ & 15 & 17 & $3+15$ & $3+14$ & 134 & ơ vi & $2+31$ & 56 & $\Xi$ \\
\hline 1013 & $1 \cdot 52$ & $4 \cdot 75$ & $11 \cdot 7$ & $12 \cdot 87$ & $14 \cdot 7$ & $17 \cdot 8$ & $23 \cdot 2$ & 24.5 & $27 \cdot 15$ & 15 & 16 & $\begin{array}{c}\text { Total } 19 . \\
3+16\end{array}$ & $3+16$ & 151 & 우 vi-iii & $1+32$ & 56 & \\
\hline $\mathbf{I} 014$ & $1 \cdot 63$ & $4 \cdot 9$ & $12 \cdot 0$ & $13 \cdot 2$ & $15 \cdot 05$ & $18 \cdot 15$ & $23 \cdot 45$ & $24 \cdot 7$ & $27 \cdot 55$ & 14 & 16 & $3+16$ & $2+15$ & 167 & 오 vi & $1+33$ & 56 & \\
\hline
\end{tabular}




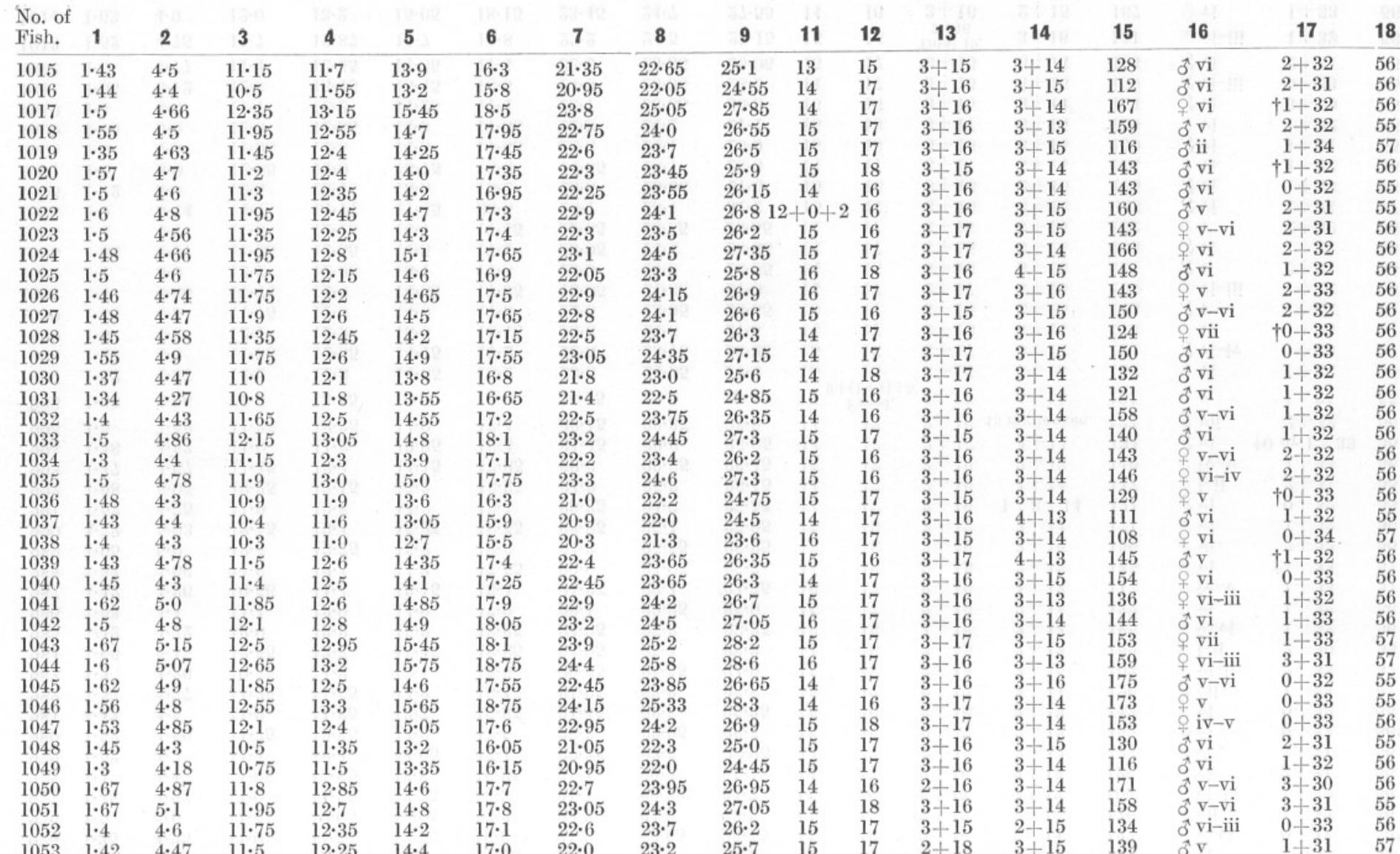




\begin{tabular}{|c|c|c|c|c|c|c|c|c|c|c|c|c|c|c|c|c|}
\hline 1054 & $1 \cdot 65$ & $4 \cdot 77$ & $11 \cdot 75$ & $12 \cdot 45$ & $14 \cdot 7$ & $17 \cdot 4$ & $22 \cdot 7$ & 23.95 & $26 \cdot 7$ & 15 & 17 & $3+17$ & $3+14$ & 161 & $\hat{O} v$ & $1+32$ \\
\hline 1055 & $1 \cdot 53$ & $4 \cdot 78$ & $12 \cdot 35$ & $13 \cdot 35$ & $15 \cdot 25$ & $18 \cdot 45$ & $23 \cdot 95$ & $25 \cdot 15$ & $27 \cdot 95$ & 14 & 17 & $3+15$ & $3+14$ & 192 & ㅇ․ & $\uparrow 0+33$ \\
\hline 1056 & $1 \cdot 66$ & $4 \cdot 98$ & $12 \cdot 05$ & $13 \cdot 4$ & $15 \cdot 05$ & $17 \cdot 9$ & $23 \cdot 25$ & $24 \cdot 55$ & $27 \cdot 25$ & 14 & 16 & $3+17$ & $3+14$ & 163 & 우 vi & $1+32$ \\
\hline 1057 & 1.4 & $4 \cdot 3$ & $10 \cdot 9$ & $11 \cdot 7$ & $13 \cdot 5$ & $16 \cdot 15$ & $21 \cdot 35$ & $22 \cdot 5$ & $25 \cdot 05$ & 14 & 17 & $3+17$ & $3+14$ & 129 & ơ vi & $2+31$ \\
\hline 1058 & $1 \cdot 57$ & $4 \cdot 65$ & $11 \cdot 2$ & $12 \cdot 05$ & $14 \cdot 0$ & $16 \cdot 7$ & $22 \cdot 0$ & $23 \cdot 1$ & $25 \cdot 85$ & 14 & 17 & $3+17$ & $3+14$ & 136 & q $\mathbf{v}$ & $2+31$ \\
\hline 1059 & $1 \cdot 53$ & $4 \cdot 57$ & $11 \cdot 5$ & $12 \cdot 05$ & $14 \cdot 3$ & $17 \cdot 15$ & $22 \cdot 55$ & $23 \cdot 75$ & $26 \cdot 45$ & 14 & 17 & $3+16$ & $3+16$ & 171 & $0^{1} \mathrm{v}$ & $1+32$ \\
\hline 1060 & $1 \cdot 47$ & 4.5 & 11.55 & $12 \cdot 2$ & $14 \cdot 25$ & $17 \cdot 15$ & $22 \cdot 3$ & $23 \cdot 55$ & $26 \cdot 25$ & 14 & 16 & $3+16$ & $3+14$ & 144 & q $\mathbf{v i}$ & $1+32$ \\
\hline 1061 & $1 \cdot 4$ & $4 \cdot 48$ & $11 \cdot 4$ & $11 \cdot 75$ & $14 \cdot 25$ & $17 \cdot 0$ & $22 \cdot 15$ & $23 \cdot 35$ & $25 \cdot 85$ & 15 & 18 & $3+16$ & $3+13$ & 134 & 우 $\mathbf{v}$ & $0+32$ \\
\hline 1062 & $1 \cdot 57$ & $4 \cdot 6$ & $11 \cdot 0$ & $11 \cdot 3$ & $13 \cdot 85$ & $15 \cdot 95$ & $21 \cdot 0$ & $22 \cdot 2$ & $24 \cdot 6$ & 14 & 17 & $2+17$ & $4+13$ & 128 & $\sigma^{t} \mathrm{v}-\mathrm{vi}$ & $2+33$ \\
\hline 1063 & $1 \cdot 5$ & $4 \cdot 93$ & $12 \cdot 2$ & $13 \cdot 3$ & $15 \cdot 0$ & $18 \cdot 05$ & $23 \cdot 5$ & $24 \cdot 8$ & $27 \cdot 6$ & 13 & 16 & $3+16$ & $3+15$ & 146 & o vi & $2+32$ \\
\hline 1064 & $1 \cdot 6$ & $4 \cdot 8$ & $11 \cdot 8$ & $12 \cdot 2$ & $14 \cdot 5$ & $17 \cdot 3$ & $22 \cdot 6$ & $23 \cdot 8$ & $26 \cdot 2$ & 13 & 16 & $3+17$ & $4+15$ & 139 & oi 1 & {$\left[1+1^{*}+30\right]$} \\
\hline 1066 & $1 \cdot 43$ & $4 \cdot 4$ & $11 \cdot 05$ & $11 \cdot 9$ & $14 \cdot 05$ & $16 \cdot 95$ & 9 & $23 \cdot 0$ & $25 \cdot 5$ & 15 & 16 & $3+$ & $3+14$ & 116 & o vi-iii & $1+32$ \\
\hline 1067 & $1 \cdot 7$ & $5 \cdot 08$ & $12 \cdot 5$ & $13 \cdot 2$ & $15 \cdot 5$ & $18 \cdot 6$ & $23 \cdot 85$ & $25 \cdot 2$ & $28 \cdot 1$ & 14 & 16 & $3+17$ & $3+14$ & 154 & o iv-v & $3+31$ \\
\hline 1068 & $1 \cdot 54$ & $4 \cdot 6$ & $11 \cdot 35$ & $12 \cdot 45$ & $14 \cdot 35$ & $17 \cdot 1$ & $22 \cdot 1$ & $23 \cdot 3$ & $25 \cdot 75$ & 14 & 15 & $3+16$ & $3+14$ & 129 & 우 vi-iv & $0+33$ \\
\hline 1069 & 1.5 & $4 \cdot 44$ & $10 \cdot 45$ & $11 \cdot 1$ & $13 \cdot 0$ & $15 \cdot 75$ & $20 \cdot 6$ & $21 \cdot 7$ & $24 \cdot 2$ & 14 & 16 & $3+16$ & $3+15$ & 113 & ㅇ vii & $1+31$ \\
\hline 1070 & $1 \cdot 42$ & $4 \cdot 37$ & $10 \cdot 95$ & $11 \cdot 35$ & $13 \cdot 4$ & $16 \cdot 2$ & $21 \cdot 1$ & $22 \cdot 2$ & 24.55 & 16 & 17 & $3+16$ & $3+14$ & 129 & ơ vi & $\uparrow 2+31$ \\
\hline 1071 & $1 \cdot 45$ & $4 \cdot 2$ & $10 \cdot 3$ & $10 \cdot 85$ & $12 \cdot 7$ & $15 \cdot 45$ & $20 \cdot 2$ & $21 \cdot 25$ & $23 \cdot 75$ & 15 & 16 & $3+16$ & $3+15$ & 117 & ơvi & $1+31$ \\
\hline 1072 & 1.7 & $5 \cdot 1$ & $12 \cdot 7$ & $13 \cdot 7$ & $15 \cdot 8$ & $18 \cdot 8$ & $24 \cdot 2$ & $25 \cdot 63$ & $28 \cdot 65$ & 15 & 16 & $3+16$ & $2+15$ & 182 & q vi & $1+32$ \\
\hline 1073 & $1 \cdot 3$ & $4 \cdot 2$ & $10 \cdot 6$ & $11 \cdot 35$ & $13 \cdot 3$ & $15 \cdot 9$ & $20 \cdot 9$ & $22 \cdot 05$ & $24 \cdot 6$ & 14 & 17 & $3+16$ & $2+15$ & 127 & $\sigma^{*} \mathrm{v}-\mathrm{vi}$ & $0+33$ \\
\hline 1074 & 1.5 & $4 \cdot 72$ & $11 \cdot 7$ & $12 \cdot 35$ & $14 \cdot 45$ & $17 \cdot 5$ & $22 \cdot 6$ & $23 \cdot 85$ & $26 \cdot 6$ & 15 & 16 & $3+16$ & $2+14$ & 121 & o $\mathrm{ii}$ & $3+31$ \\
\hline 1075 & $1 \cdot 47$ & $4 \cdot 7$ & $11 \cdot 8$ & $12 \cdot 55$ & $14 \cdot 45$ & $17 \cdot 7$ & $22 \cdot 55$ & $23 \cdot 75$ & $26 \cdot 55$ & 15 & 16 & $3+15$ & $3+14$ & 147 & & $\uparrow 0+32^{*}$ \\
\hline
\end{tabular}




\section{APPENDIX TO TABLES.}

$\dagger$ This sign in all cases indicates that no lateral processes are present in the vertebræ immediately preceding the 1st vertebra with complete hæmal arch or the most anterior incomplete arch denoted in the records. The processes in these cases have been cleaned away.

* This sign on the measurements (Column 1 to 9 ) indicates that the character is slightly abnormal in some way or that the part measured is slightly damaged. In columns 17 and 18 the asterisks refer to the following :-

Fish No.

\section{Sample III.}

$3 * 12$ th vertebra with complete hæmal arch has two psirs of hæmal arches, but only one extra neural process on the right, which is attached to the 11th neural arch. The vertebra itself is slightly longer than adjacent ones, but has been counted as and appears to be only one. The left hæmal process of the 15th vertebra with complete hæmal arch is attached to the 14th hæmal arch and its fellow is free. The hæmal processes of the next vertebra, the 16 th, are also free.

$9 *$ Right portion of 11 and 12 hæmal arches fused with left division of 11 th hæmal spine. 12th, left portion of hæmal arch free.

$34 *$ 19th vertebra is very long and carries two pairs of neural and hæmal arches, the extra arches arising from the middle. It is recorded as one, but is apparently made up of two fused.

98 * 9th and 10th vertebræ (with complete hæmal arch) have each two pairs of neural spines, the 10th has also two pairs of hæmal spines, the abnormal pair having one limb (the left) arising from the middle of the centrum, but the other arising near the origin of the right member of the 11th hæmal arch. These vertebræ are each recorded as one; they are of normal size, and neither appears to be composed of two vertebræ fused, as is undoubtedly the case in other skeletons.

$125 *$ 2nd vertebra with well-developed ventrolateral processes (paired).

$139 * 6$ th hæmal arch with extra spine arising from junction of processes.

$157 *$ Processes missing almost entirely one side and entirely on other side.

$161 *$ Anterior group of vertebræ lost.

241 * 1st hæmal arch has a supernumerary hæmal process in the middle of the vertebra on the left side.

$243 *$ 1st hæmal arch broken, but still attached to vertebra.

$267 *$ Incomplete arch, although nearly complete. The vertebra preceding had a complete hæmal arch as noted in the record.

$270 *$ Transverse bars nearly joined. The right-hand figure of Fig. 3 is drawn from this vertebra.

$277 * 10$ th and 11th hæmal arches are joined by a bony cross-piece. 
$288 * 14$ th and 15 th vertebræ have each two pairs of hæmal spines, the subsidiary pair being in the middle, but only the 14th pair has also a corresponding neural arch.

$291 * 19$ th neural spines quite free.

$299 * 24$ th hæmal spines free; no loop formed.

$310 * 5$ th neural spines are separate, and there is an extra free neural spine on the right, and the 5th, 7th, and 8th hæmal arch processes are bifurcated at the tip, and three of the 7 th are not joined together at all, that is both spines are free.

$316 * 15$ th and 16th left rays of hæmal arches are joined up with the 15th right hæmal arch ray. The 16 th right ray is free.

351 * In the case of high numbers as 33 and 34 the first hæmal arch is generally without the lateral processes.

$352 *$ 11th hæmal arch, processes not fused, and right one bifurcated.

$401 *$ Atlas with pair of well-developed dorso-lateral spines.

416 * Extra spine on 16th hæmal arch, arising from junction of processes of arch.

427 * Last vertebra but two is peculiar, being unusually small, and being somewhat fenestrated on the left side but normal on the right side. Otherwise the skeleton is quite normal.

$435 *$ The eleventh vertebra bears on the left a hæmal spine showing a slight bifurcation at the tip; the hæmal spines in the anterior region in this fish were very well developed.

$453 * 1$ st arch broken, but still attached to vertebra.

$462 *$ Less well developed than processes of anterior vertebra.

$529 *$ The last vertebra but two is apparently made up of two fused, being almost twice as long as adjacent ones ; it bears two pairs of hæmal and neural spines, but has only an anterior and posterior articulation; the centre is distinctly fused. This is only counted as one.

$546 * 1$ st vertebra with complete hæmal arch has a supernumerary hæmal process on the left side, and the 14th vertebra with complete hæmal arch has a supernumerary pair of hæmal and neural spines arising from middle of vertebra ; both pairs of hæmal spines are joined by a cross-piece on the right side.

\section{Sample IV.}

$552 *$ 1st hæmal arch broken off centrum, but still attached.

$580 *$ Extra spine from junction of hæmal spines of 23 rd vertebra from posterior end.

$582 *$ On some posterior hæmal arches are spherical concretions which appear like little beads of melted metal. This phenomenon has been noticed on other skeletons also.

$588 *$ Atlas, 1st, and axis, 2nd, vertebræ fused together; counted as two. 
631 * This one incomplete, but with an internal process on the right hæmal process. The arch of the vertebra anterior to it is complete.

$664 * 7$ th vertebra from posterior end has two pairs of hæmal and neural spines, is longer than normal and apparently equal to two fused vertebræ. It is counted, however, as one.

$667 * 4$ th, 5 th, and 6 th vertebræ from posterior end have been broken and recovered at some period of life of the fish.

687 * The last vertebra bears three pairs of neural and two pairs of hæmal spines, it is nearly twice as long as a normal one, and has a thickening in the middle of the centrum where apparently two vertebræ have fused. It is undoubtedly two vertebræ fused. It is, however, only counted as one in the table.

$709 * 3$ rd complete arch has a tiny extra connecting hæmal process on the left.

711 * Middle tips of hæmal arches with curious concretionary appearance, just as though the arches were of metal and had been melted in parts.

$740 *$ The right hæmal process of 28 th vertebra is joined to junction of hæmal processes of 27 th vertebra ; the left process of the 28 th vertebra being free.

746 * 13th vertebra from anterior end is about half as long again as normal, and has ridges around its middle indicating fusion of two vertebræ. It is, however, counted as one.

There is no normal articulation in the middle, but complete fusion.

$767 * 9$ th vertebra very long and apparently two vertebræ fused, similar to 704. It is counted, however, as one.

$782 *$ It is quite possible that one or more arches are missing here ; possibly too well cleaned.

784 * Arch incomplete, though the one anterior to it is complete (broken, but still attached to centrum).

$803 *$ 1st hæmal arch broken, but still attached to vertebra.

$841 *$ There is nothing unusual in the appearance of this skeleton.

$843 *$ 1st hæmal arch with a rib attached.

$855 *$ 2nd, 3rd, 4th, 5th, and 6th rays of anal fin anastomising.

897 * In the region two-thirds from anterior end the hæmal and neural arches arise from abnormal positions and there are a few extra spines.

899 * Several hæmal arches with processes interchanged,i.e. processes on opposite sides not joined up with fellow, but with those anterior or posterior to them.

911 * 3rd hæmal arch broken, but was undoubtedly complete.

921 * 46th vertebra from anterior end, 3 pairs of hæmal and $2 \frac{1}{2}$ pairs of neural spines. The centrum is abnormally long, nearly twice normal length, and is apparently equal to at least 2 vertebræ fused. It is counted as one. 
925 * In Character 17 the " $a$ " portion is recorded hence first in order to avoid any bias towards making up the number to the apparent total of 33 or 34 potential arches.

$943 * 35$ th vertebra with two pairs of neural and hæmal spines, nearly twice as long as normal, and apparently equals two fused. Counted as one.

$962 *$ Arches about middle of skeleton a little abnormal.

$975 * 12$ th vertebra nearly twice as long as normal, and apparently equal to two fused, but only counted as one. Also 30th vertebra with $1 \frac{1}{2}$ hæmal arches and spines, but otherwise of normal size. 32nd also with abnormal hæmal arch processes.

$980 * 39$ th vertebra with one hæmal arch attached to arch of 38 th vertebra.

$1010 * 37$ th vertebra has one extra neural and one extra hæmal spine, but is otherwise normal.

1064 * Vertebra behind 1st complete arch carries an incomplete but welldeveloped arch; it is counted with those having complete arch.

1067 * Left process of 44 th hæmal arch is joined up with junction of 43rd hæmal arch.

$1075 *$ 1st hæmal arch broken, but still attached to centrum.

Note.-The lateral processes of the vertebræ preceding those with complete hæmal arch are sometimes long without internal processes, and at other times shorter with the internal process developing. Nevertheless, the arbitrary character chosen has been adhered to as closely as possible. There are, however, doubtful cases.

Very frequently the 4th, 5th, and 6th vertebræ from posterior end have the base of the hæmal arch passing diagonally across the ventral anterior half of the centrum, and in this respect are unlike the other vertebræ. 